\title{
Controlling VR Games: Control Schemes and the Player Experience
}

\author{
by \\ Erin Martel
}

A thesis submitted to the Faculty of Graduate and Postdoctoral Affairs in partial fulfillment of the requirements for the degree of

\author{
Master of Arts \\ in \\ Human-Computer Interaction
}

Carleton University

Ottawa, Ontario

(C) 2020,

Erin Martel 


\section{Abstract}

Control schemes are central to the user experience in video games as they determine the quality of a user's interaction with the game world. Since the 1990's, desktop 3D games have adopted the "mouselook" scheme, in which the mouse simultaneously rotates the camera view, aims and steers the avatar. However, virtual reality games do not yet have a standard control scheme and require a different approach in order to integrate input from the head-mounted display, an additional input device not present in desktop 3D games. To address this gap, we conducted two mixed-methods studies to evaluate the usability of control schemes for controller-based VR games in the FPS genre. The first study used keyboard and mouse and the second used Oculus Touch motion controllers as input devices. User study 1 showed that "coupled" control schemes in which the camera view and steering the avatar were controlled by both the mouse and head-mounted display were most usable. Study 2 showed that both the coupled and "decoupled" schemes were usable, although some users found the decoupled scheme disorienting. 


\section{Acknowledgements}

First, I would like to thank my thesis supervisors, Dr. Kasia Muldner and Dr. Robert Teather for their patience and help during this process. To Dr. Muldner, I am grateful for your expert guidance and generosity in my time at Carleton. To Dr. Teather, thank you for offering the resources of your lab and for the excellent advice.

Thanks to all of my professors and to the administrative staff, especially Dr. Robert Biddle and also Dr. Bruce Tsuji, who was my first supervisor and who was a significant help during the admission process. Most of all, I would like to Dr. Anthony Whitehead for giving me the opportunity to study at Carleton. He was truly a source of support and inspiration to all. 


\section{Dissemination of this Thesis}

Chapter 2 and Chapter 3 of this thesis were previously published as part of the following peer-reviewed paper:

Chapter 2 \& Chapter 3: Martel, E., \& Muldner, K. (2017). Controlling VR

Games: Control Schemes and the Player Experience. Entertainment Computing, 21, 19-31. 


\section{Table of Contents}

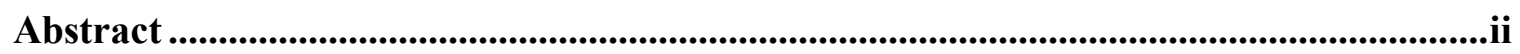

Acknowledgements ..........................................................................................................................iii

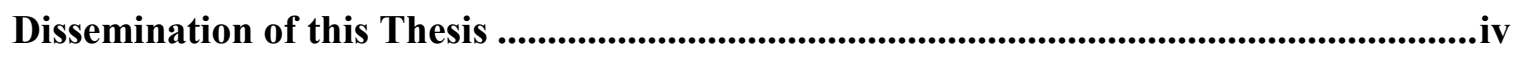

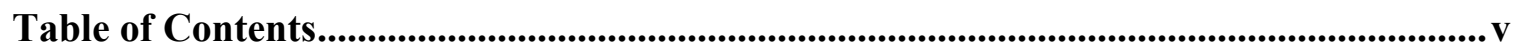

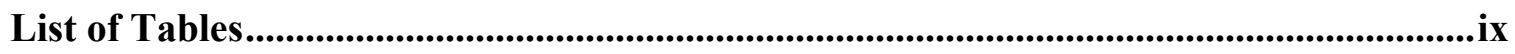

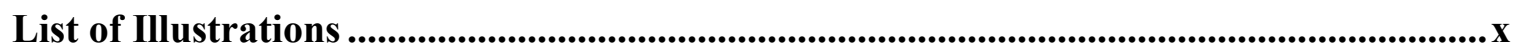

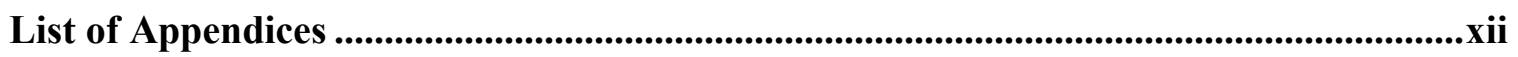

Chapter 1: Introduction ...................................................................................................................1

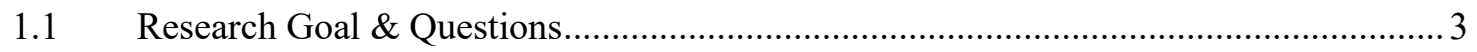

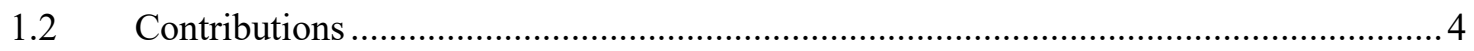

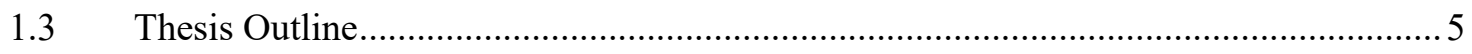

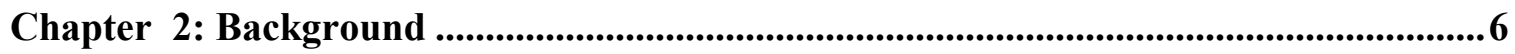

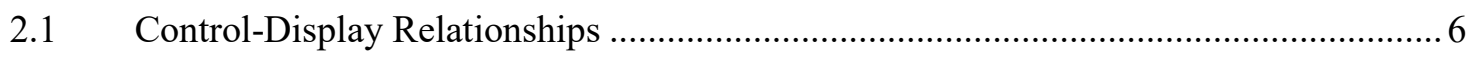

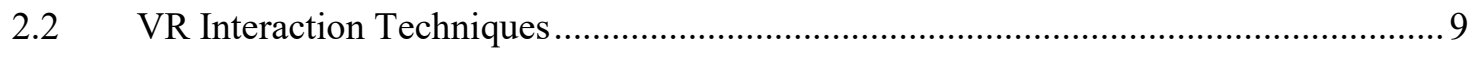

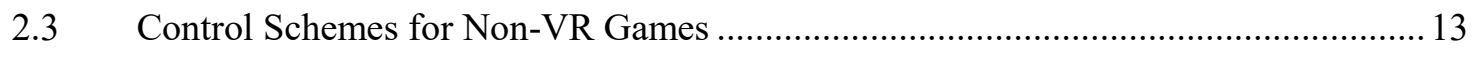

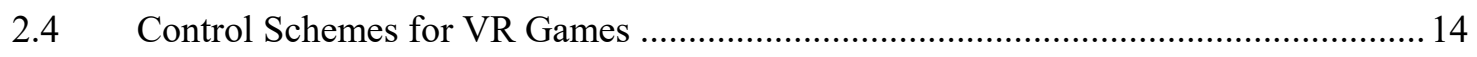

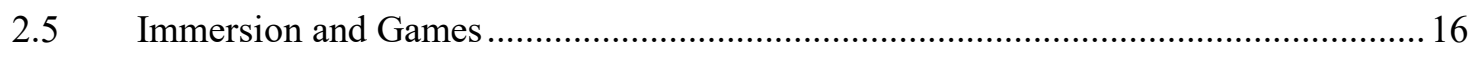

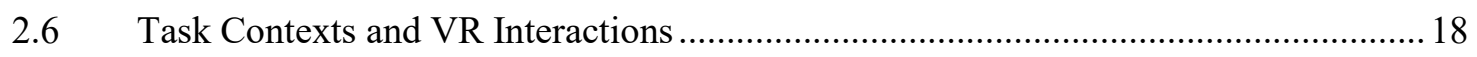

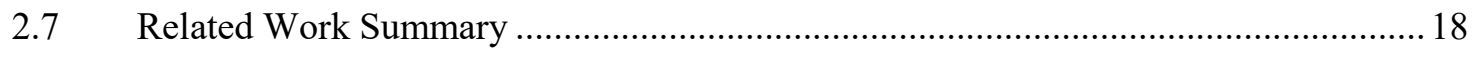


Chapter 3: User Study 1 ...........................................................................................................20

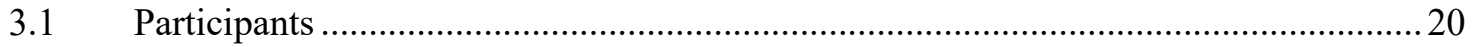

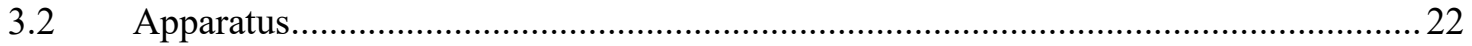

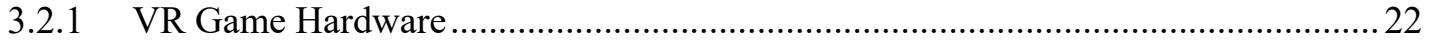

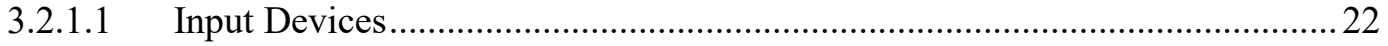

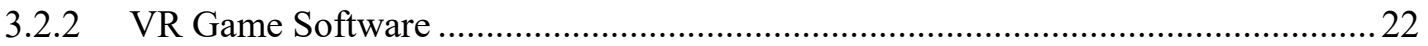

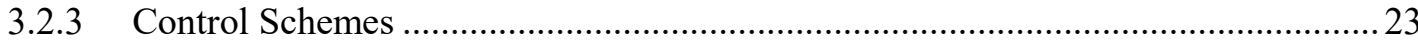

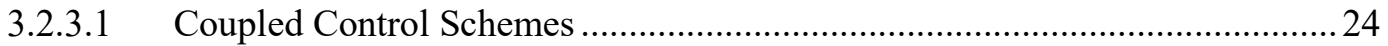

3.2.3.2 Coupled Control Schemes with a Dead Zone................................................25

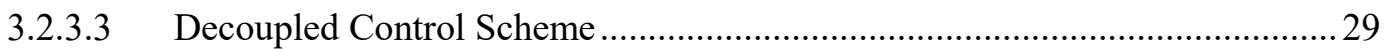

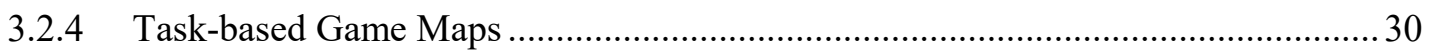

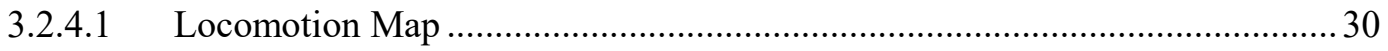

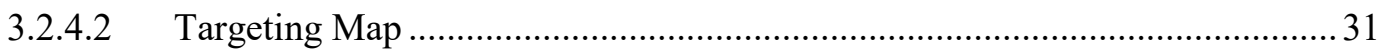

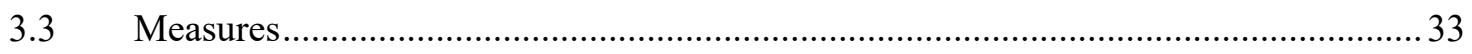

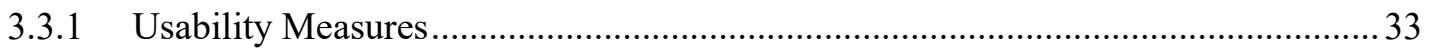

3.3.1.1 Control Scheme Preference Questionnaire …............................................... 35

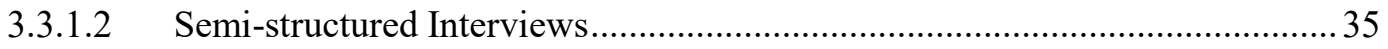

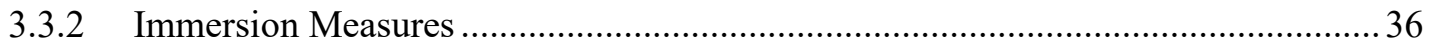

3.3.2.1 The Immersive Experiences Questionnaire ................................................. 36

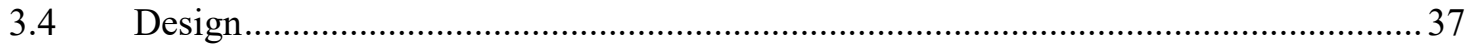

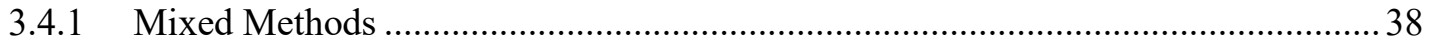

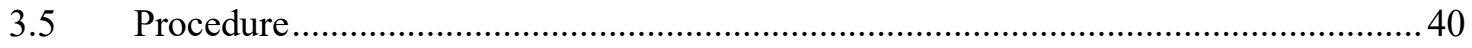

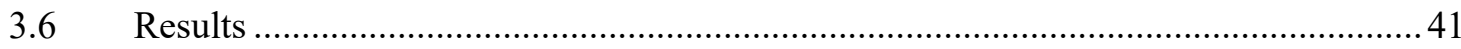

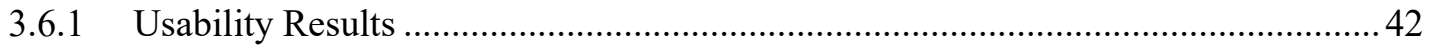

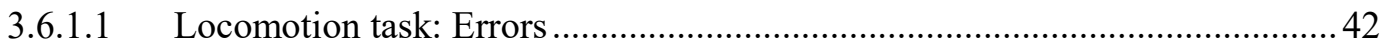

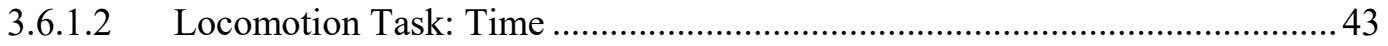




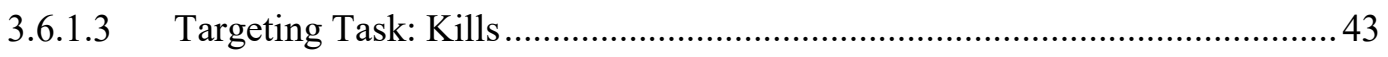

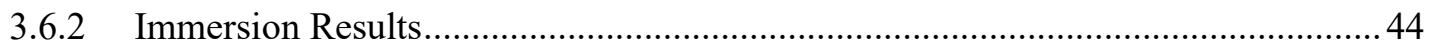

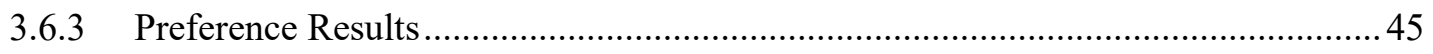

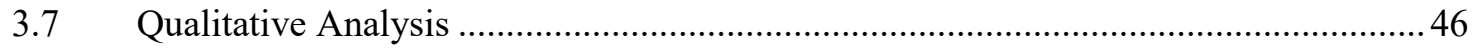

3.7.1 Coupled Controls Scheme Impressions............................................................... 47

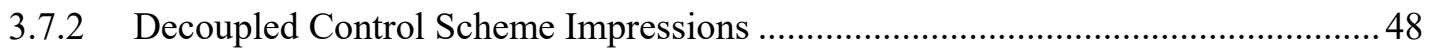

3.7.3 Dead Zone Control Scheme Impressions ............................................................ 50

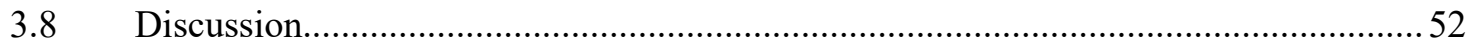

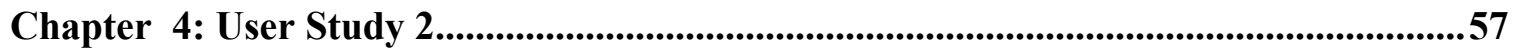

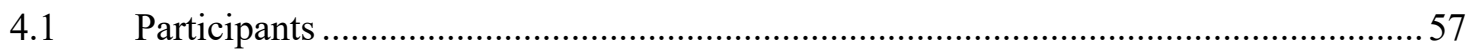

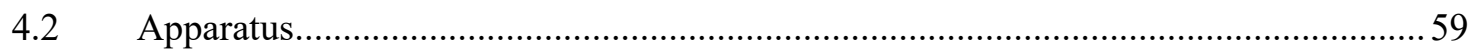

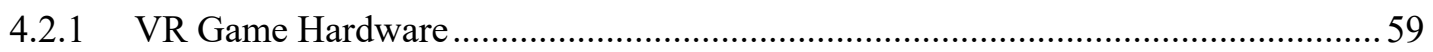

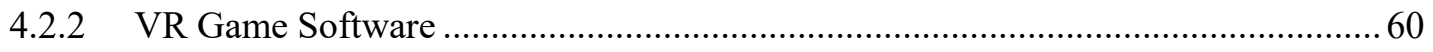

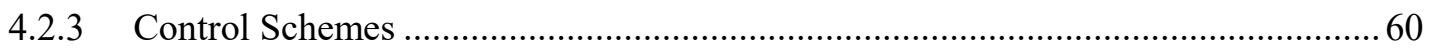

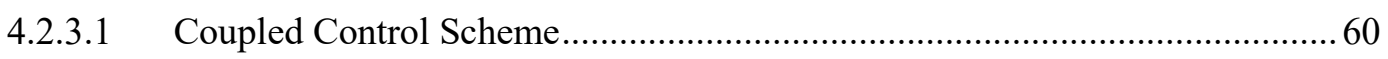

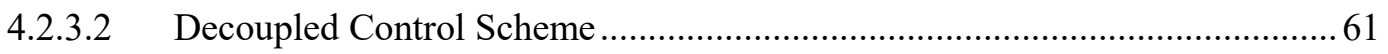

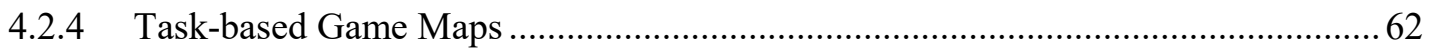

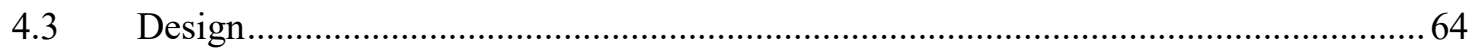

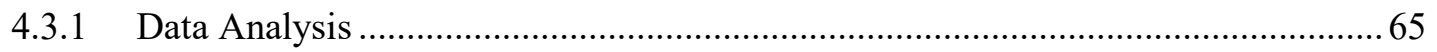

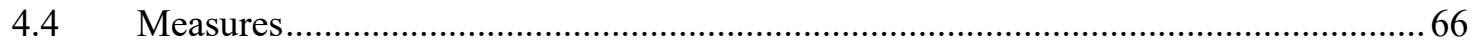

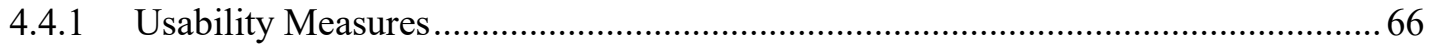

4.4.1.1 Task-specific Control Scheme Questionnaire …..........................................6 68

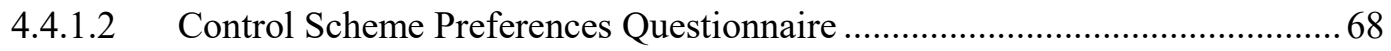

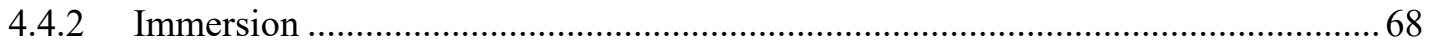

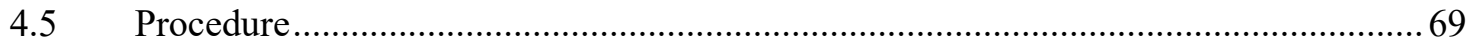

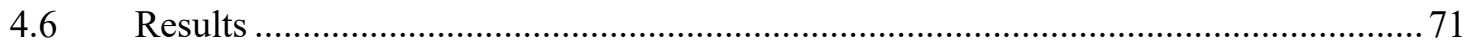




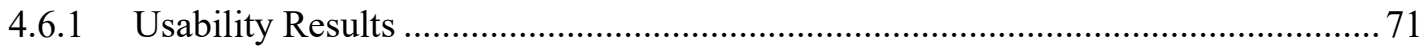

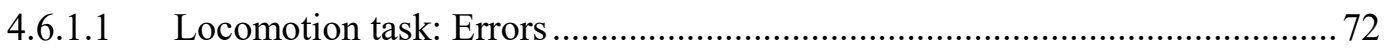

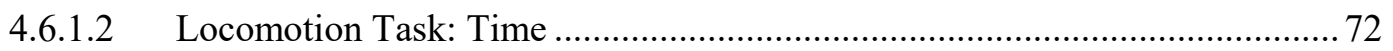

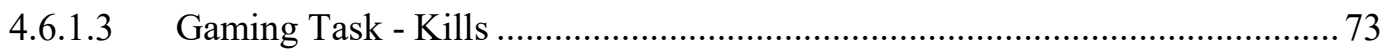

4.6.1.4 Gaming Task - Survival Time …............................................................... 73

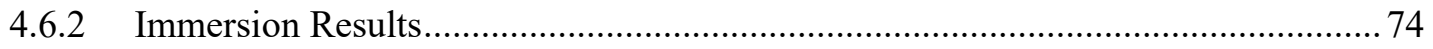

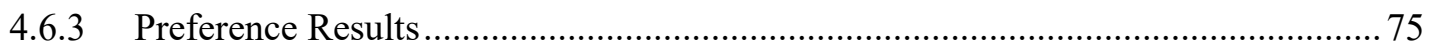

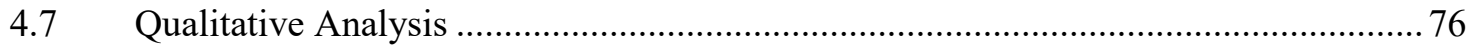

4.7.1 Coupled Controls Scheme Impressions............................................................. 77

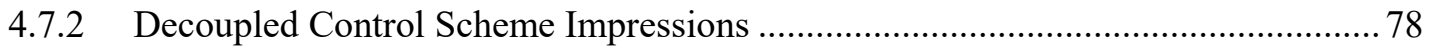

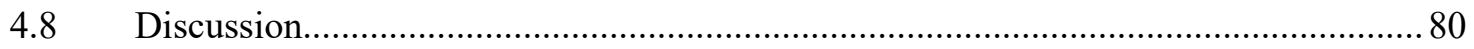

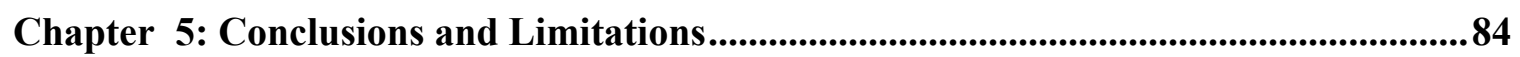

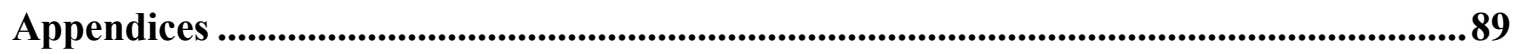

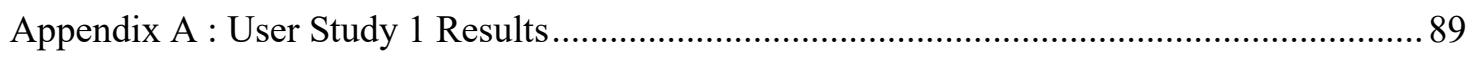

Appendix B Game Experience Questionnaire ................................................................ 91

Appendix C Control Scheme Preferences Questionnaire ................................................ 92

Appendix D Immersive Experiences Questionnaire ....................................................... 93

Appendix E Task-Specific Control Scheme Questionnaire.................................................96

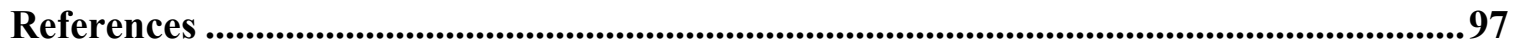




\section{List of Tables}

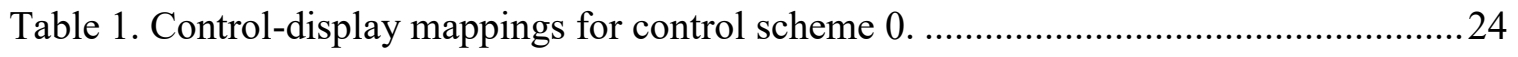

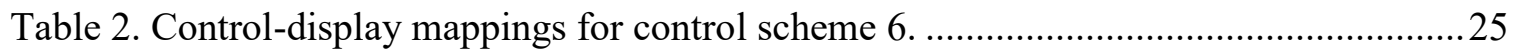

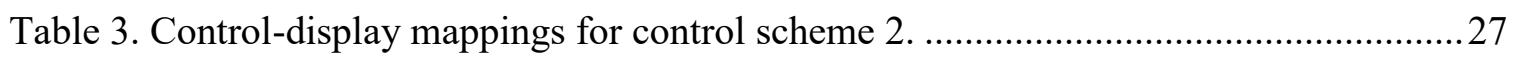

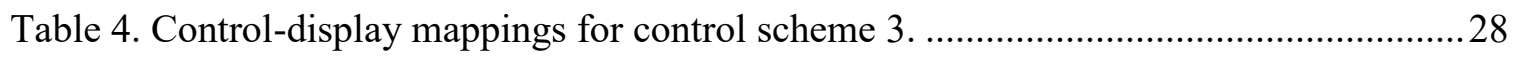

Table 5. Control-display mappings for control scheme 4. .................................................29

Table 6. Control-display mappings for control scheme 1 . ............................................. 30

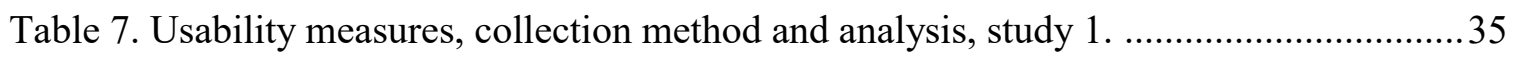

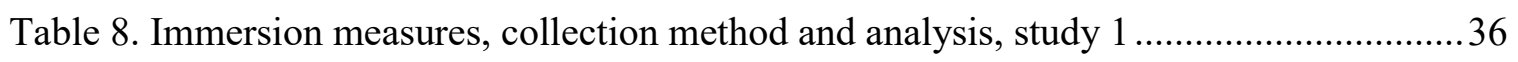

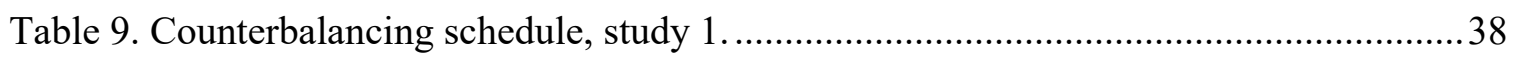

Table 10. Control-display mappings for the coupled control scheme, study 2 ....................61

Table 11. Control-display mappings for the decoupled control scheme, study $2 \ldots \ldots \ldots \ldots \ldots \ldots . . . .62$

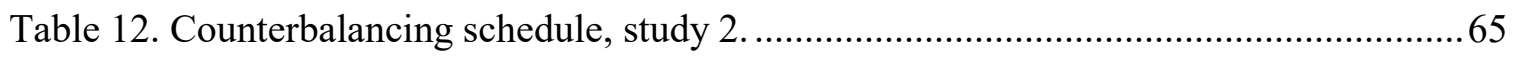

Table 13. Usability measures, collection method and data analysis, study 2 .......................67

Table 14. Immersion measures, collection method and analysis, study 2 ..........................69

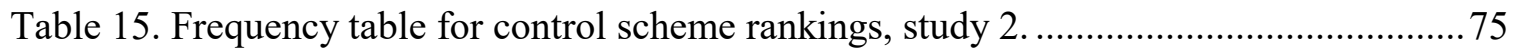

Table 16. Complete usability results for all 6 control schemes, study 1 ...........................90 


\section{List of Illustrations}

Illustration 1. HMD and sensor DOF, Oculus Rift [60] .................................................

Illustration 2. Bowman's taxonomy of VR travel interaction techniques .............................. 10

Illustration 3. Visualization of coupled and decoupled controls, reproduced from Martel et al.

Illustration 4. Boletsis and Cedergren VR Locomotion techniques typology.......................13

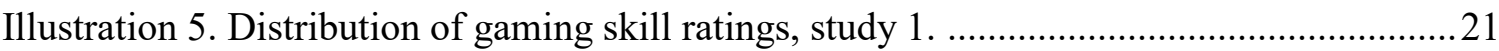

Illustration 6. Axis definitions for three-dimensional coordinate system, reproduced from

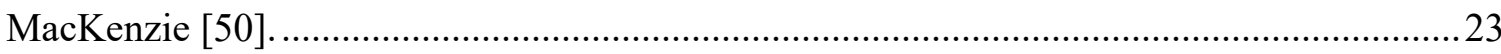

Illustration 7 Visualization of the dead zone for control scheme 2, study 1 .........................26

Illustration 8 Visualization of dead zone for control scheme 3 and 4, study $1 . \ldots \ldots \ldots \ldots \ldots \ldots . . . . .27$

Illustration 9. In-game first person view of the locomotion map........................................ 31

Illustration 10. Locomotion map in the Hammer map editor........................................... 31

Illustration 11. In-game first-person view of the targeting map, study 1 ................................ 32

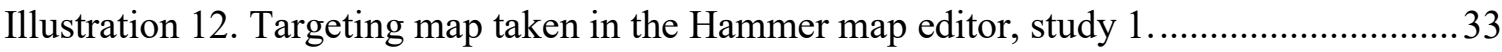

Illustration 13 Process flow for convergent parallel mixed-methods...................................39

Illustration 14 Usability results: mean errors (bottom); mean kills (middle); and mean time

(top), by control scheme type, study 1. 
Illustration 15. Mean immersion scores (top) and mean scores for the single question measure

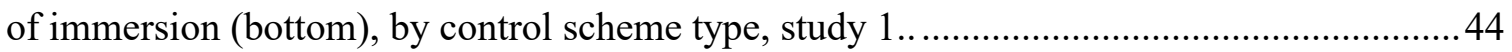

Illustration 16. Mean preference scores by control scheme type, study 1 ............................46

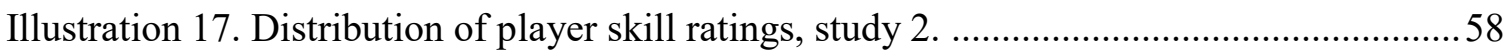

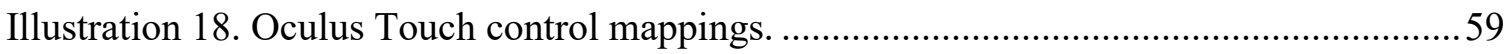

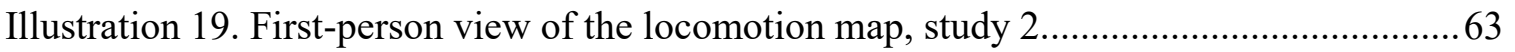

Illustration 20. Top-down view of the locomotion map from the Serious Editor..................63

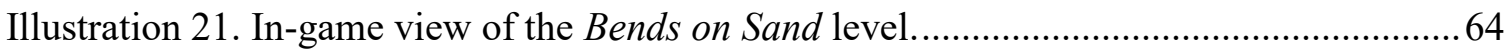

Illustration 22. Process flow for convergent parallel mixed-methods .................................66

Illustration 23. The Karnak Temple level, which participants played to acclimatize to VR...70

Illustration 24. Locomotion task mean time and errors by control scheme, study $2 \ldots \ldots \ldots \ldots . . .72$

Illustration 25. Gaming task mean survival time and kills by control scheme, study 2 .........73

Illustration 26. Mean immersion (top) and single question measure of immersion scores

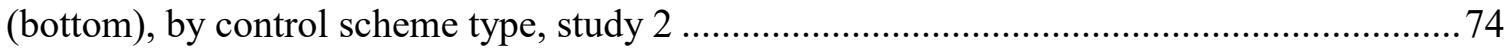

Illustration 27. Distribution of "naturalness" ratings, by control scheme. .............................76

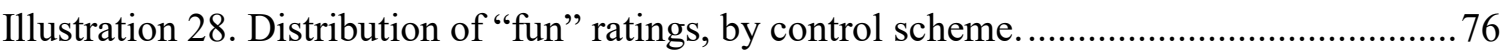




\section{List of Appendices}

Appendix A: Complete Results, Study 1................................................................89

Appendix B: Gaming Experience Questionnaire.............................................................91

Appendix C: Control Scheme Preferences Questionnaire.............................................92

Appendix D: Immersive Experiences Questionnaire..................................................93

Appendix E: Task-specific Control Scheme Questionnaire..........................................95 


\section{Chapter 1: Introduction}

Virtual reality (VR) is a medium [73] that is rapidly gaining popularity for gaming, prompted by advances in consumer-grade head mounted displays. Modern VR gaming systems are equipped with input devices that track the user's body: the headmounted display tracks head rotation; motion controllers such as the Oculus Touch track hand movements and sensors capture hand and head position within the tracked space. These input modalities must be integrated into a game's control scheme. It is possible to map these inputs to several in-game functions, such as: rotating the camera view; rotating the player avatar and aiming at targets-creating a myriad of control mapping combinations, or schemes.

The dominant control scheme for desktop $3 \mathrm{D}$ games is called "mouselook", in which the mouse rotates the player avatar and camera view simultaneously and the target selection reticule is fixed to the center of the screen. In the context of VR games, no

dominant scheme has yet emerged. To address this, designers have implemented a variety of control schemes for gaming in this medium, but few studies have investigated their impact and it is not yet clear which approach offers the best user experience. 
A key area of experimentation in the first generation of VR games is interaction techniques for locomotion [4, 88]. Botetsis and Cedergren developed a typology of the current VR locomotion techniques, identifying 4 main types: room-scale, motionbased, teleportation-based and controller-based. Many current VR games use teleportation-based interaction technique $[5,7,15,30]$. Studies show that the pointto-teleport technique reduces cybersickness [25], as do other teleport-based locomotion techniques such as translation snapping or dash locomotion [24], and out-of-body locomotion [32], making it suitable for games in many genres. While this is an advantage, there are other usability considerations. An advantage of controller-based locomotion (often called "full locomotion" in the game industry) identified in the literature is that the technique uses the continuous viewpoint movement, which allows players to maintain visual contact with threats in the game world $[3,56]$, and allows continuous avatar representation for multiplayer gamers [32]. Consequently, many VR first-person shooter (FPS) games use controller-based locomotion, which is furthermore most similar to traditional mouselook controls, because users move through virtual world in response to continuous input on a joystick or other input device.

In this thesis, we evaluate control schemes for controller-based locomotion in firstperson shooter (FPS) games. We identified two common control scheme types for controller-based FPS VR games: coupled and decoupled. In coupled control schemes, the player always moves in the direction the camera is facing. In decoupled schemes, the player can look around the environment freely without affecting the direction of movement. Early VR research in non-gaming contexts found that coupled control schemes resulted in better performance $[10,66]$. However, these studies may not be 
generalizable to modern gaming contexts, as modern gamers may have become acclimatised to the dominant "mouselook" control scheme for desktop $3 \mathrm{D}$ games. Prior to this thesis work [51], a comparison of coupled and decoupled control schemes for controller-based VR games did not exist.

\subsection{Research Goal \& Questions}

Control schemes are fundamental to our interaction with games [48], but little is known about the usability of control schemes for controller-based locomotion in VR FPS games. A comprehensive survey of VR locomotion technique studies since 2014 showed that "since the VR revival, the focus of VR locomotion research has been on VR technology and various technological aspects, overshadowing the investigation of user experience." [4] As a step towards addressing this gap, we conducted two studies to compare the usability of coupled and decoupled control schemes. The first study used mouse + keyboard as input devices and compared six different VR control schemes for the VR port of the game Half Life 2: Deathmatch [80]. Our second study used the Oculus Touch motion controls as input devices and compared two different control schemes for the VR port of Serious Sam VR: The First Encounter [21]. We followed a mixed-methods approach for both studies, measuring the effect of control schemes with usability metrics and also gathering qualitative feedback.

Our goal was to identify the factors affecting control scheme usability and provide recommendations for creating usable control schemes for FPS VR games. To reach this goal, this thesis focuses on the following research questions:

R1: Does control scheme affect usability measures (effectiveness, efficiency, satisfaction and learnability)? 
Hypothesis 1: The coupled control scheme will have highest scores on effectiveness, efficiency, learnability and satisfaction, based on prior VR research $[10,56,65]$.

R2: Does control scheme affect game immersion scores?

Hypothesis 2: The decoupled scheme will have highest immersion scores. Research in control schemes for desktop 3D games show that gestural [1, 19, $43,55,57,67]$ and head-tracking input $[36,41,68,69,83]$ are more immersive than traditional gamepad or mouse + keyboard and were preferred despite having a negative effect on performance.

R3: Does task context have an effect on usability or immersion scores?

Hypothesis 3: The coupled scheme is better for all tasks, although task context has been shown to affect control scheme usability for certain game genres $[38,41]$.

R4: What key control scheme attributes affect control scheme usability?

Hypothesis 4: Schemes that are more similar to existing game controls and thus more familiar to "gamers", such as the coupled scheme, will be more usable.

\subsection{Contributions}

The work described in this thesis contributes to our understanding of control schemes for controller-based VR games in the FPS genre. 
Theoretical Contribution:

- We provide empirical evaluation of VR control scheme usability and immersiveness.

Practical Contribution:

- The results of our study can provide rationale for designing control schemes based on user feedback, rather than solely on task performance.

\subsection{Thesis Outline}

This thesis has 5 chapters, beginning with the Introduction in Chapter 1. Chapter 2 relates the history of control schemes for desktop $3 \mathrm{D}$ and VR games as well as reviewing the literature on interaction techniques for locomotion in virtual reality.

In Chapters 3 and 4, we describe two mixed-methods studies that evaluate the usability of virtual reality control schemes for VR games. Study 1 using keyboard and mouse as input devices and the study 2 using the Oculus Touch motion controls.

This thesis concludes with a summary of our findings, a discussion of the limitations of our study and possible directions for future work. 


\section{Chapter 2: Background}

\subsection{Control-Display Relationships}

We begin with foundational concepts related to interaction between users and computer systems. Within the HCI literature, interactions are governed controldisplay relationships, where a user's manipulation of an input device (a physical controller such as a keyboard or a mouse) produces a visual or auditory response. As per Scott MacKenzie [49]:

"Control-display relationships are sometimes called mappings, since the relationships attribute how a controller property maps to a display property."

Input devices are capable of sensing properties such as position, displacement ("the amount of movement along an axis" [50], also called "motion") and the amount of force. These properties can be sensed along three linear and three angular dimensions, which are called the degrees of freedom (DOF), as seen in Illustration 1. For example, a mouse senses displacement with 2 degrees of freedom: translation on the $\mathrm{x}$-axis and translation on the z-axis. Device input can be mapped in spatially congruent ways, such as mouse translation on the $\mathrm{x}$ - 
axis moving the cursor along the display's x-axis; or incongruously, as when mouse translation on the $\mathrm{z}$-axis is mapped to moving the cursor along a display's y-axis. The ability to creatively map input to display is often required by the inherent limitations of input devices, and is at the heart of control scheme design.

The properties of an input device may make them more or less useable for a given task. In this thesis, we use mouse and keyboard as input devices in user study 1 and Oculus Touch motion controllers for user study 2. Both studies also use the Oculus Rift head-mounted display and its external sensors. Below we describe the sensing properties of each input device used in our study and research on their advantages and limitations.

Mouse. User study 1 uses standard mouse as the input device to rotate the camera view, the avatar. A mouse senses displacement with 2-DOF along the xaxis and z-axis. The mouse is more accurate than joystick or head-mounted display for targeting tasks in gaming studies [31, 40, 59], as well as in Fitt's law studies $[64,76]$.

Keyboard. User study 1 also uses a keyboard as input to initiate forward (W key), backwards (S key), strafing-or sideways movement-left (A key) and strafing right (S key) in relation to the avatar's facing direction. Keyboard keys are discrete input.

Motion controllers and joysticks. User study 2 uses the Oculus Touch motion controller, a 6 DOF position-sensing device. The motion controller's position and orientation is mapped in a spatially-congruent, 1:1, relationship to 
corresponding position and orientation within the game. Targeting objects in the game is achieved by "pointing" the Oculus Touch controller toward the desired target.

Each controller also has an integrated elastic-isometric joystick that senses displacement with 2 DOF along $\mathrm{x}$-axis and $\mathrm{z}$-axis, similar to a mouse, albeit with less precision due to employing velocity or rate-control mappings [31, 40, $59]$.

Head mounted display. Oculus Rift's DK2 HMD (used in study 1) and CV1 HMD (used in study 2) are display devices, but are also position-sensing devices that include integrated and external sensors to provide 6-DOF tracking and 1:1 mapping of the user's head position and orientation, as seen in Illustration 1. As per Oculus' PC SDK Developer Guide:

"The Oculus Rift hardware contains...a gyroscope, accelerometer, and magnetometer. There is also a sensor to track headset position. The information from each of these sensors is combined through the sensor fusion process to determine the motion of the user's head in the real world and synchronize the user's view in real-time." [6o]

Note that the DK2 had a single positional sensor with a limited tracking volume $72^{\circ} \mathrm{H}$ $\mathrm{x} 52^{\circ} \mathrm{V}$, which is approximately 8 feet and too small for "roomscale" VR. The CV1 has two sensors that each have a tracking volume of $100^{\circ} \mathrm{H} \times 70^{\circ} \mathrm{V}$. Our testing environment tracked an area of $8 \times 8$ feet. 

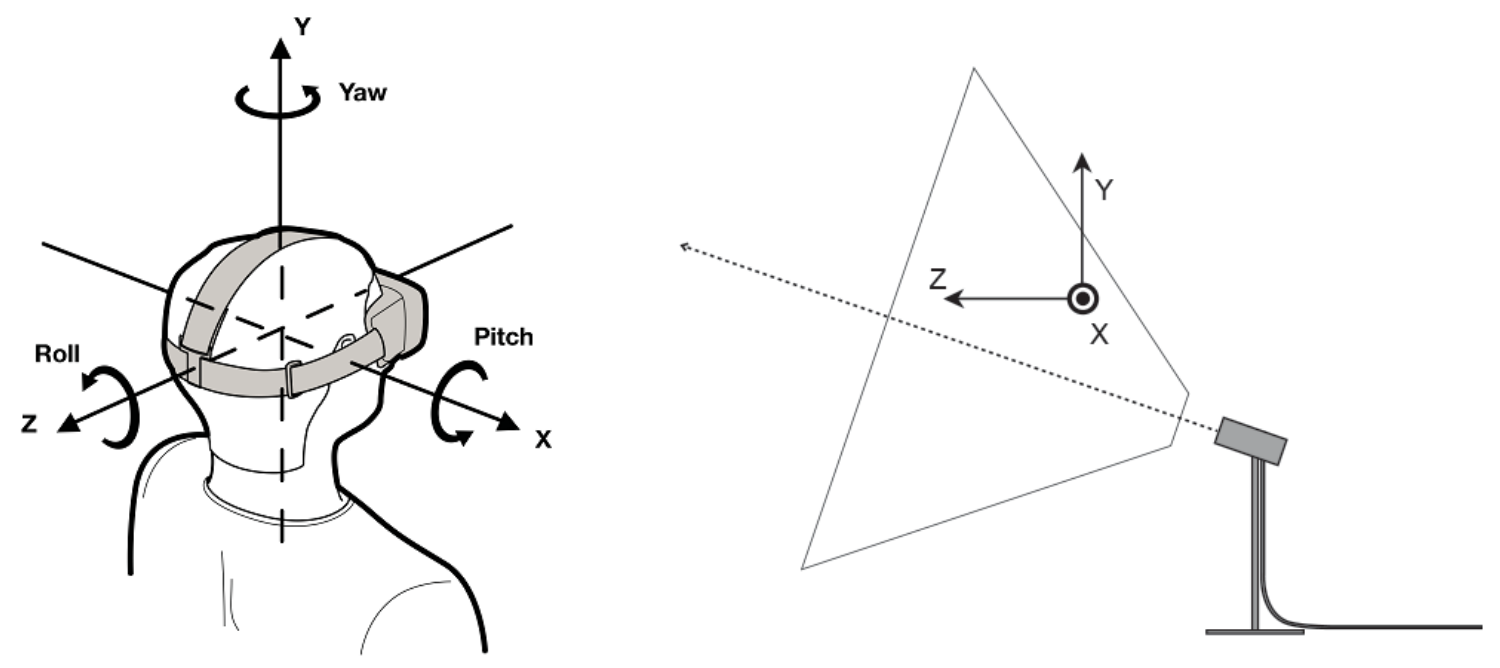

Illustration 1. HMD and sensor DOF, Oculus Rift [60]

Studies have investigated the impact of head tracking on players for desktop $3 \mathrm{D}$ games, but not virtual reality games. Wang et al. [83], Zhou et al. [89], Ilves et al [36] and Sko et al. [68] all found that head-tracking resulted in higher ratings of enjoyment and immersion, but was not conducive to precision. Kulshreshth et al. had similar findings but also found head tracking aided in threat identification [41].

\subsection{VR Interaction Techniques}

Interaction techniques are the "method by which the user performs a task on a computer via the user interface" [9]. They consist of the device input, the mappings of device input to display outputs and the feedback provided to the user.

In this section we describe work on interaction techniques for virtual environments in non-gaming contexts. Bowman et al. $[8,13,14]$ created a taxonomy of interaction techniques for two activities common in virtual environments: travel, or locomotion ("the movement of the user's viewpoint from place to place") and selection ("indicating virtual objects within the environment"). By classifying the various 
techniques, Bowman et al. provided a framework that could be used to create novel interaction techniques by combining various input devices and control-display mappings. See reproduction of Bowman's taxonomy for VR travel in Illustration 2 [13].

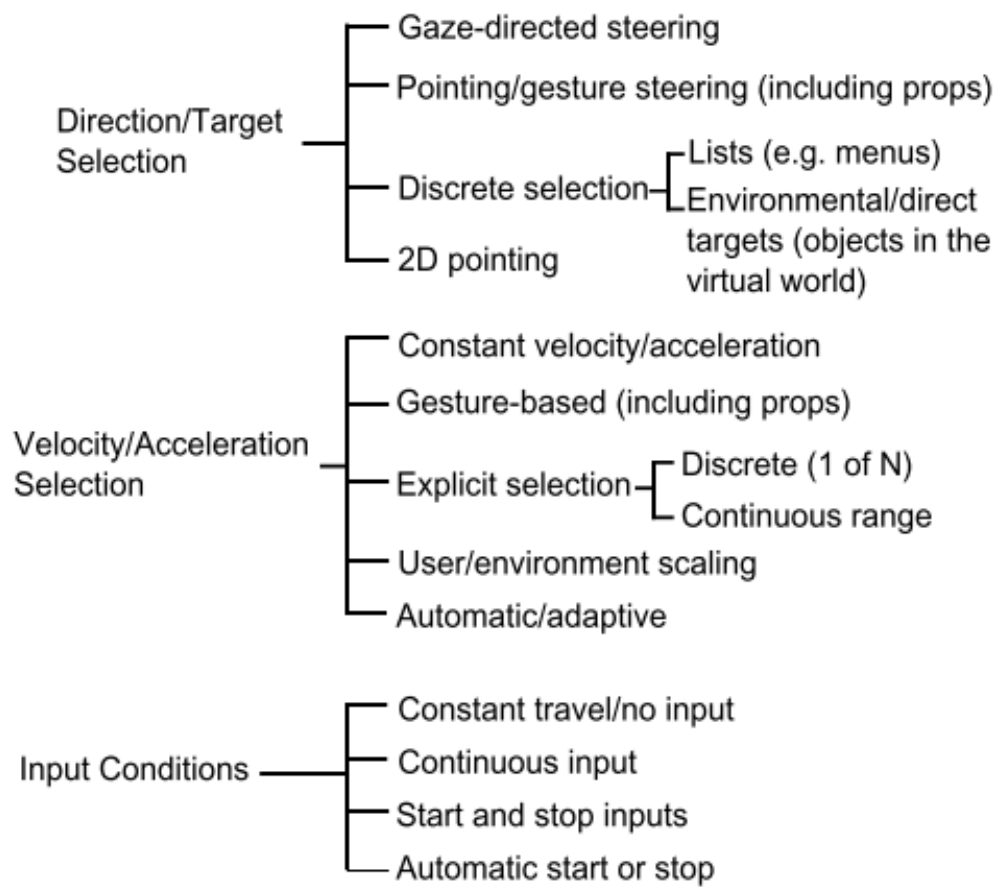

\section{Illustration 2. Bowman's taxonomy of VR travel interaction techniques}

Bowman's travel taxonomy included "gaze-directed" direction selection (or "steering"), gesture steering, discrete selection of targets, and 2D pointing. In the present study, we are evaluating control schemes that use the modern equivalent of two of these: (1) the gaze-directed technique, which we call "coupled"; and (2) the 2D pointing technique, which we call "decoupled".

Gaze-directed steering is a technique in which the HMD is mapped to control both the camera view and direction selection, as shown in Illustration 3 [51]. The gesture 
and 2D pointing schemes are decoupled: the HMD controls the camera, while the pointer or gesture steers. Note that there are no commonly agreed upon names for these interaction techniques. Gaze-directed $[9,56]$ steering has also been referred to as "head-coupled" [9], "view-directed" [66] and "head-directed" [29]. 2D pointingsteering has been referred to as "non-head-coupled" [9], "hand-directed" [56] and "de-coupled" [66]. We have chosen coupled and decoupled as our terms.
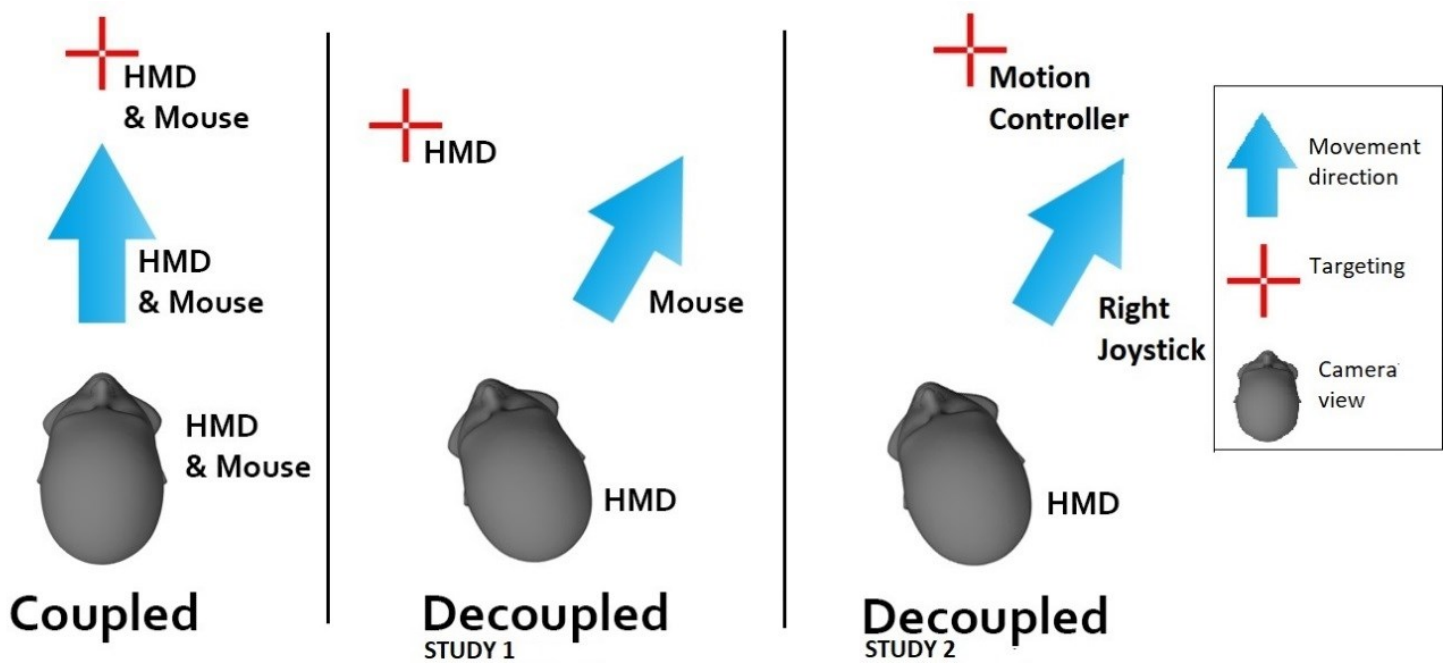

Illustration 3. Visualization of coupled and decoupled controls, reproduced from Martel et al.

The most often researched VR interaction techniques for travel were coupled and decoupled. As Bowman states:

"Most techniques differ only in the direction or target specification subtask, and several common technique components are listed in the taxonomy. Gaze-directed steering uses the orientation of the head for steering, while pointing gets this information from the user's hand.” [11]

Early VR researchers theorized that coupled travel would be most natural for VR applications [56]. Bowman et al. [10] conducted two experiments that contrasted "gaze-directed" travel, with steering using a 2D pointing device. Study one tested 
how fast participants could move to a target object. The effect of control scheme on time was not significant. Note that this study only measured efficiency, whereas our study will also test effectiveness using error counts to see how accurately a participant's can move with the control schemes. In study 2, Bowman et al. found that coupled "gaze-directed" travel was significantly faster for moving to positions relative to a target object, for example, moving to a point in front of a target. Relative positioning is useful to gain a view of a target object, which could have applications in gaming to gain an advantageous position relative to enemies. Ruddle [65] found that coupled travel that used head tracking to control movement direction (coupled or "gaze-directed" travel) was most effective as compared to other schemes. For targeting tasks, Lampton et al. [42] found that head tracking was the most effective input for targeting. Pausch et al. [63] obtained similar results in their study that compared HMD and joystick as input devices for locating targets in a virtual world.

More recently, Boletsis and Cedergren [6] created a typology of the current VR locomotion techniques based on a literature review of techniques studied between 2014 and 2017. The review highlighted that these existing studies compare the usability of different interaction techniques, but none evaluate the usability of distinct control schemes for a technique [5, 7, 30, 39, 58, 77]. Illustration 4 , reproduced from Boletsis and Cedergren [6] shows a visual breakdown of the typology of locomotion techniques. These techniques are discussed in more detail in the Control Schemes for VR Games section of this chapter. 


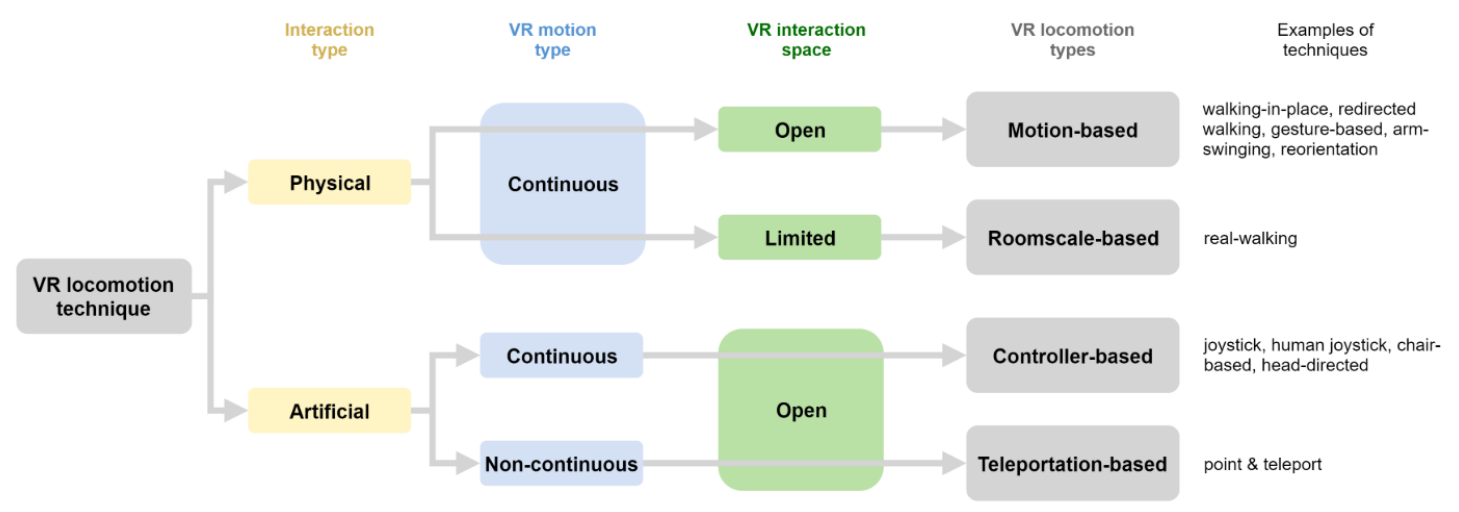

Illustration 4. Boletsis and Cedergren VR Locomotion techniques typology.

\subsection{Control Schemes for Non-VR Games}

A control scheme refers to the control-display mappings in a given interaction technique. In other words, the manner in which a game maps data received from an input device to in-game functions. The term "control scheme" is primarily used by game developers, as games are intensely interactive and require a complex set of control-display mappings [34]. For desktop 3D games, control schemes typically allow the player to affect the following in-game functions related to locomotion (listed below):

- Rotating the in-game camera view, in order to look around the game world.

- Rotating the avatar, or "steering", in order to specify the direction of movement through the game world.

- Targeting objects in the environment. For example, moving a selection cursor such as a crosshair to target an enemy in a FPS game. 
Gaming control schemes have evolved with each leap forward in gaming technology [45]. In the $2 \mathrm{D}$ gaming era, player characters or camera viewpoint moved using a fixed set of possible directions (i.e., up/down/left/right), for which a keyboard or directional pad input device was perfectly adequate. When $3 \mathrm{D}$ games emerged, a new scheme was needed to enable looking and moving through a three-dimensional game world with increased granularity. Thus, the now-established "mouselook" control scheme was introduced. This scheme was developed for PC games in the mid-1990's, invented for first-person shooter games such as Marathon [18] and Quake [35]. The control scheme is called mouselook because moving the mouse along the $\mathrm{x}$ - and $\mathrm{z}$ axis is mapped to rotation of the camera view, targeting, and steering around the $y$ and $\mathrm{x}$-axis, respectively:

"Orienting the player in a first-person game requires methods to control both the position and the view-direction of the player in the world. These two facets of orientation are generally controlled with two different sets of device inputs. On a personal computer, players typically delegate keyboard keys to movement, and the mouse to controlling the view...Console games have two analog joysticks to control both movement and view." [40]

Mouselook was later adapted to console controllers (using an analog stick in place of the mouse) and remains the standard control scheme for desktop 3D games.

\subsection{Control Schemes for VR Games}

In the VR context, the challenge with control scheme design is how to integrate additional input devices, such as the HMD and motion controllers. Most current- 
generation consumer-grade VR gaming systems, such as the Oculus Rift and the HTC Vive are equipped with positional tracking for roomscale-based locomotion-which supports physical movement around the tracked environment, but only within a small area-thus requiring other techniques to allow the player to move longer distances in the virtual environment.

Current VR games have adopted a variety of interaction techniques to supplement roomscale-based locomotion. The point-to-teleport technique is frequently used, where the player chooses a target location, by pointing a ray emitted from the controller, and their point of view jumps to that location $[5,7,15,30]$. The related translation snapping (or dash) [28] and out-of-body [32] locomotion techniques also feature non-continuous movement. Controller-based locomotion offers continuous movement and is often used in VR first-person shooters. The gameplay in FPS demands that you are always aware of the position of fast-moving enemies in your vicinity, so controller-based locomotion is often used in these game genres to avoid losing connection with the game world $[3,56]$. In this study, we are evaluating control schemes for controller-based locomotion in FPS VR games.

For controller-based games, there are two main approaches to control scheme design: coupled schemes and decoupled schemes, which are also prevalent in nongaming contexts as described in the VR Interaction Techniques section above. As defined earlier, with coupled control schemes, the player's camera view and movement direction are coupled, so the user moves in the direction the camera is facing. Thus, this control scheme is closest to mouselook. The rotation of the camera viewpoint and movement direction is mapped to both the mouse (or right joystick) and the HMD. Targeting is either fixed to the center of the camera view or can be 
decoupled from the camera view with the addition of another input device, such as the Oculus Touch motion controls we use in study 2. In contrast, decoupled schemes allow the player to look around the environment freely without affecting movement direction. In decoupled schemes, targeting enemies or objects in the virtual environment is sometimes fixed to the center of the camera view and therefore headtracking is the physical control, as seen in user study 1's control scheme 1. However, targeting can also be decoupled from the camera view as in the case of some of the experimental "dead zone" control schemes in study 1, or in study 2 where the motion controllers were used for targeting. In subsequent chapters, we will describe in more detail the specific control schemes we are using for user study 1 (Chapter 3) and user study 2 (Chapter 4).

In a preliminary pilot study, we evaluated five VR control schemes for the FPS controller-based locomotion game Team Fortress 2 [52]. Player performance was highest using the coupled control scheme, but immersion scores were highest with the decoupled scheme. In this thesis, we will use a mixed methods approach that includes a qualitative data component in order to probe for reasons behind the empirical results.

\subsection{Immersion and Games}

Immersion is a key factor influencing the user experience in video games. Immersion has been defined as being "concerned with the specific, psychological experience of engaging with a computer game" [37]. This definition diverges from VR researchers concept of immersion, which Lombard \& Ditton describe as: "the extent to which the senses are engaged by the mediated environment" $[44,70]$. 
The present thesis focuses on game immersion rather than VR immersion. Researchers have identified several sources of game immersion. For instance, Mayra and Ermi's model [54] includes challenge, sensory, and imaginative sources. Brown and Cairns [16] found that players experience immersion when the game controls are effortless, but the game still requires concentration to master (similar to Mayra and Ermi's challenge factor). Jennett and Cox [37] isolated five factors that lead to the experience of immersion, including cognitive involvement, emotional involvement, real-world dissociation, challenge, and control.

VR games promote sensory immersion because they block out the real world and are viewed through a HMD with a large field of view (FOV). Sensory immersion in a gaming context is similar to VR immersion, but can also be achieved with desktop $3 \mathrm{D}$ games. A human's natural FOV is 180 degrees, and while the Oculus Rift's FOV is considerably less (at 100 degrees), it is close enough make the user to feel like they are "inside" the virtual environment and are viewing the scene as they would in real life. Indeed, studies have verified that VR games are more immersive than desktop 3D games. Yoon et al. [86] modified Unreal tournament 2004 to use head tracking and a data glove. Immersion was measured using both physiological (heart rate) and self-report methods for VR and desktop $3 \mathrm{D}$ versions of the game; both measures demonstrated that the VR version resulted in higher immersion. Tien Tan et al. [74] found that a VR version of Half-Life 2 promoted higher immersion than a desktop $3 \mathrm{D}$ version of the game. As a final example, two studies by Lugrin et al. [46, 47] compared performance and enjoyment in a VR and desktop $3 \mathrm{D}$ versions of Unreal Tournament, and showed that the VR version was superior for these variables. 
To date, research has focused on demonstrating that VR games are more immersive than desktop $3 \mathrm{D}$ games, rather than focusing on the factors that affect immersion within the VR medium. To address this gap, here we study the impact of the type of control scheme on immersion.

\subsection{Task Contexts and VR Interactions}

The early VR research discussed so far found that interaction techniques perform differently depending on the interaction task [2, 42, 56, 84]. Bowman et al. [12, 13] propose using a testbed approach in which a set of the most common VR interaction techniques are evaluated in custom-made test environments to isolate the effect of tasks on interaction technique usability. The testbed approach of Bowman et al. was inspired by the research by Lampton et al. [42]. Volbracht et al. [82] categorized the many factors that can influence the efficacy of an interaction technique based on Bowman's taxonomy: the virtual environment, task, user experience, motion control and navigation strategy or aids.

As the effectiveness of an interaction technique is influenced by the task context, in our work we follow a similar method and evaluate two VR tasks common in FPS games, locomotion and targeting. We also evaluated control schemes in an ecologically valid gaming context task, namely a first-person shooter game level that combined targeting and locomotion tasks.

\subsection{Related Work Summary}

To date, VR games research has focused on identifying the strengths and weaknesses of the VR medium as compared to desktop 3D. This research has established that VR 
is in general more immersive, albeit more difficult to control because of the imprecise nature of head tracking and other natural tracked input. With the rise of consumer VR, an explosion of new and old locomotion techniques for VR have been adopted, and several usability studies have compared them empirically $[5,7,30,39$, 58, 77]. However, prior work has not investigated the impact of different control schemes for VR games, and the existing research on VR control schemes needs to be updated for gaming-specific contexts and experienced gamers. Thus, it is not clear how a given control scheme impacts the player experience, performance, and immersion-information needed to design VR games that are usable and immersive. To address this gap, in the present thesis we will analyze how variations in control schemes affect the user experience within the VR medium within a game context. 


\section{Chapter 3: User Study 1}

We now describe study 1 , which evaluated three virtual reality control schemes types: coupled, decoupled and dead zone control schemes, using keyboard and mouse as input devices. We measured the effect of control scheme type on usability and immersion.

This chapter was published as part of "Controlling VR Games: Control Schemes and the Player Experience" in Entertainment Computing [53].

\subsection{Participants}

Thirty eight participants were recruited through posters, social media, and word-ofmouth ${ }^{1}$. Two participants did not complete the study because of cybersickness (two males). Thus, the analysis is based on data from 36 participants (28 male). The mean age of the participants was 24; the range was $19-40$.

\footnotetext{
${ }^{1}$ Data collection and preliminary analysis were completed as part of a directed study. The preliminary data analysis was later extended and written up in the form of a journal article for publication. The preliminary analysis is therefore substantially different from what is shown in this thesis.
} 
Our participants needed to have the in-depth knowledge of games in order to understand the differences between the control schemes. Thus, to control for experience level, we recruited participants who self-identified as gamers. Prospective participants were asked to rate their skill in playing $3 \mathrm{D}$ games on a scale of 1 to 5 (with 5 being very skilled) in answer to the question: "What is your skill level in playing games set in a 3D world from a first person viewpoint." The mean skill level for the sample was 3.7. See Illustration 5 .

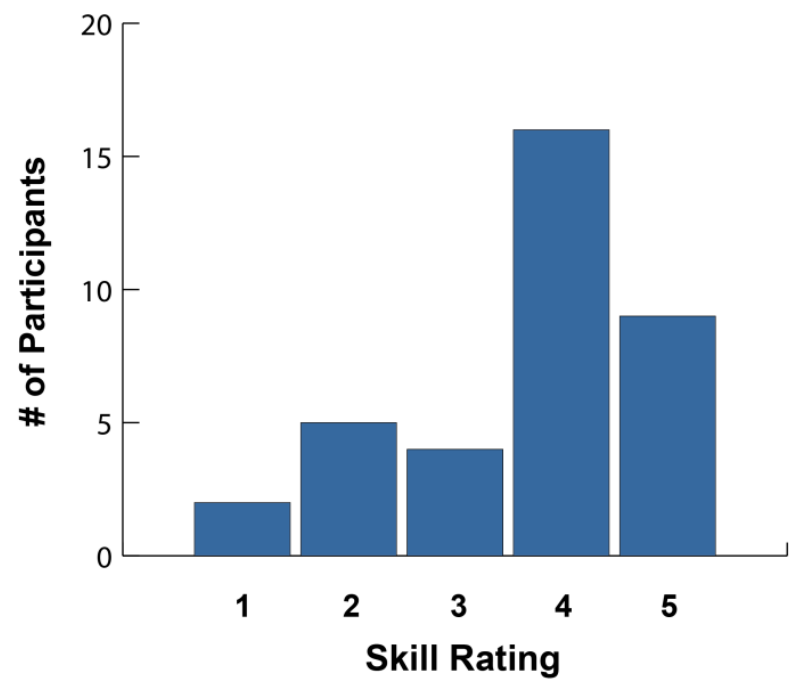

Illustration 5. Distribution of gaming skill ratings, study 1 .

We also collected information about the platforms that participants typically used to play games. Thirty one of the participants had experience with PC games using keyboard and mouse. Only five participants had no experience gaming with the mouse and keyboard (i.e., they were console gamers or PC gamers who used a gamepad as an input device). Six participants had played a VR game before--only two had used the Oculus rift, and the remaining four had played location-based VR games from 15-20 years ago during the first wave of VR gaming. 


\subsection{Apparatus}

\subsubsection{VR Game Hardware}

For study 1, we used a PC gaming setup that would be familiar to gamers, which included a standard mouse and keyboard attached to a Dell XPS 8700 with 17-inch monitor. To this standard gaming system we added an Oculus Rift Development Kit 2 [62]-a HMD designed for VR gaming. The Rift's screen shows a stereoscopic image that gives the user a wider field of view (110 degrees horizontally) than possible with desktop 3D games. The device also tracks head rotation and translation on the $\mathrm{x}, \mathrm{y}$ and $\mathrm{z}$ axes.

\subsubsection{Input Devices}

We used the mouse and keyboard as additional input devices. These are standard for PC gaming and will be familiar to game players. As control schemes were the focus of our study, we avoided introducing another layer of novelty by using non-standard controllers. Mouse and keyboard have the additional advantage of being characterized by increased accuracy and speed as compared to other input devices such as gamepads, wands, gesture and positional tracking [31, 40, 59].

\subsubsection{VR Game Software}

Study 1 used the game Half-Life 2: Deathmatch (HL2:DM) [80], a first-person shooter game by Valve Software. HL2: DM meets the requirements of our study because it (1) includes the Source SDK [81] and its Hammer Editor game authoring tool, which was needed for building the custom VR environments that we used in the study; and (2) it comes with pre-defined VR control schemes. 


\subsubsection{Control Schemes}

We categorized the VR control schemes that are available in HL2: DM into three types: coupled schemes, coupled schemes with a "dead zone", and decoupled schemes. We refer to the control schemes using the naming conventions in HL2: DM options menu.

In this section, we will explain how the each control scheme functions. We have also provided tables that show the control-display mappings for each input axis, showing the relationship between each control and display degree-of-freedom, for each component of the locomotion interaction technique. For example, in Table 1, for Viewpoint Control, mouse movement in the $x$-axis is mapped to viewpoint rotation around the vertical axis (i.e., $\ominus y$ ). In other words, moving the mouse left and right makes the viewpoint rotate left and right. Illustration 6 shows the axis definitions for our coordinate system:

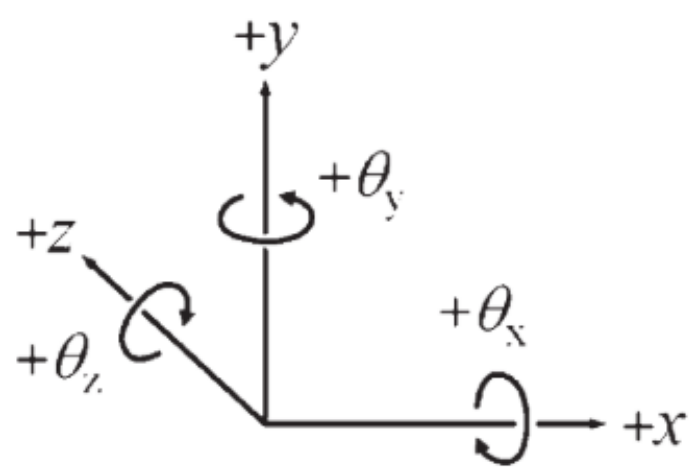

Illustration 6. Axis definitions for three-dimensional coordinate system, reproduced from MacKenzie [50]. 


\subsubsection{Coupled Control Schemes}

With the coupled control schemes, the HMD input is coupled to the mouse so that they control the same functionalities. Consequently, the player can choose to use either the mouse or the HMD (or both as they see fit). There are two coupled control schemes in HL2:DM, which are called control scheme o and 6 in the options menu.

Control Scheme o. With control scheme o, the HMD and the mouse both control the camera view, steering, and targeting, but this scheme constrains the mouse rotation around the $\mathrm{x}$ axis (pitch). Moving the mouse cursor up and down does not affect the camera view or move the targeting reticule, so the player must use the HMD to look up. Note that movement direction only needs to be mapped to rotation around the y axis for all control schemes. See Table 1.

\begin{tabular}{|c|c|c|c|c|c|c|c|c|c|c|c|}
\hline \multicolumn{4}{|c|}{ Viewpoint Control } & \multicolumn{4}{|c|}{ Movement Direction } & \multicolumn{4}{|c|}{ Targeting } \\
\hline \multirow{2}{*}{$\begin{array}{l}\text { Input } \\
\text { Device }\end{array}$} & \multicolumn{3}{|c|}{ Control-Display Mappings } & \multirow{2}{*}{$\begin{array}{l}\text { Input } \\
\text { Device }\end{array}$} & \multicolumn{3}{|c|}{ Control-Display Mappings } & \multirow{2}{*}{$\begin{array}{l}\text { Input } \\
\text { Device }\end{array}$} & \multicolumn{3}{|c|}{ Control-Display Mappings } \\
\hline & Axis & Control & Display & & Axis & Control & Display & & Axis & Control & Display \\
\hline \multirow[t]{6}{*}{ Mouse } & $x$ & & & \multirow[t]{6}{*}{ Mouse } & $x$ & & & \multirow[t]{6}{*}{ Mouse } & $x$ & & \\
\hline & $y$ & & & & $y$ & & & & $y$ & & \\
\hline & $z$ & & & & $z$ & & & & $z$ & & \\
\hline & $\theta_{x}$ & & & & $\theta_{x}$ & & & & $\theta_{x}$ & & \\
\hline & $\theta_{y}$ & & • & & $\theta_{y}$ & & b & & $\theta_{y}$ & & $\checkmark$ \\
\hline & $\theta_{z}$ & & & & $\theta_{z}$ & & & & $\theta_{z}$ & & \\
\hline \multirow[t]{6}{*}{ HMD } & $x$ & & $\longrightarrow$ & \multirow[t]{6}{*}{ HMD } & $x$ & & & \multirow[t]{6}{*}{ HMD } & $x$ & $\bullet$ & $\longrightarrow$ \\
\hline & $y$ & $\bullet$ & $\longrightarrow$ & & $y$ & & & & $y$ & $\bullet$ & $\longrightarrow$ \\
\hline & $z$ & & $\longrightarrow$ & & $z$ & & & & $z$ & & $\longrightarrow$ \\
\hline & $\theta_{x}$ & & $\longrightarrow$ & & $\theta_{x}$ & & & & $\theta_{x}$ & & $\longrightarrow$ \\
\hline & $\theta_{y}$ & $\bullet$ & $\longrightarrow$ & & $\theta_{y}$ & 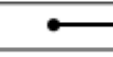 & $\longrightarrow$ & & $\theta_{y}$ & $\bullet$ & $\longrightarrow$ \\
\hline & $\theta_{z}$ & $\bullet$ & $\longrightarrow$ & & $\theta_{z}$ & & & & $\theta_{z}$ & $\bullet$ & $\longrightarrow$ \\
\hline
\end{tabular}

Table 1. Control-display mappings for control scheme 0.

Control Scheme 6. Like in control scheme o, in control scheme 6 the HMD and the mouse both control the camera view, steering and targeting, but this scheme 
constrains mouse input's rotation around the $\mathrm{x}$ axis (pitch) only for viewpoint control. See Table 2.

\begin{tabular}{|c|c|c|c|c|c|c|c|c|c|c|c|}
\hline \multicolumn{4}{|c|}{ Viewpoint Control } & \multicolumn{4}{|c|}{ Movement Direction } & \multicolumn{4}{|c|}{ Targeting } \\
\hline \multirow{2}{*}{$\begin{array}{l}\text { Input } \\
\text { Device }\end{array}$} & \multicolumn{3}{|c|}{ Control-Display Mappings } & \multirow{2}{*}{$\begin{array}{l}\text { Input } \\
\text { Device }\end{array}$} & \multicolumn{3}{|c|}{ Control-Display Mappings } & \multirow{2}{*}{$\begin{array}{l}\text { Input } \\
\text { Device }\end{array}$} & \multicolumn{3}{|c|}{ Control-Display Mappings } \\
\hline & Axis & Control & Display & & Axis & Control & Display & & Axis & Control & Display \\
\hline \multirow[t]{6}{*}{ Mouse } & $x$ & & & \multirow[t]{6}{*}{ Mouse } & $x$ & & & \multirow[t]{6}{*}{ Mouse } & $x$ & & \\
\hline & $y$ & & & & $y$ & & & & $y$ & & \\
\hline & $z$ & & & & $z$ & & & & $z$ & & \\
\hline & $\theta_{x}$ & & & & $\theta_{x}$ & & & & $\theta_{x}$ & & $\bullet$ \\
\hline & $\theta_{y}$ & & b & & $\theta_{y}$ & & $\checkmark$ & & $\theta_{y}$ & & b \\
\hline & $\theta_{z}$ & & & & $\theta_{z}$ & & & & $\theta_{z}$ & & \\
\hline \multirow[t]{6}{*}{ HMD } & $x$ & & $\longrightarrow$ & \multirow[t]{6}{*}{ HMD } & $x$ & & & \multirow[t]{6}{*}{ HMD } & $x$ & & $\longrightarrow$ \\
\hline & $y$ & $\bullet$ & $\longrightarrow$ & & $y$ & & & & $y$ & & $\longrightarrow$ \\
\hline & $z$ & $\bullet$ & $\longrightarrow$ & & $z$ & & & & $z$ & & $\longrightarrow$ \\
\hline & $\theta_{x}$ & & & & $\theta_{x}$ & & & & $\theta_{x}$ & & \\
\hline & $\theta_{y}$ & $\bullet$ & $\longrightarrow$ & & $\theta_{y}$ & $\bullet$ & $\longrightarrow$ & & $\theta_{y}$ & $\bullet$ & $\longrightarrow$ \\
\hline & $\theta_{z}$ & $\bullet$ & $\longrightarrow$ & & $\theta_{z}$ & & & & $\theta_{z}$ & $\bullet$ & $\longrightarrow$ \\
\hline
\end{tabular}

Table 2. Control-display mappings for control scheme 6.

\subsubsection{Coupled Control Schemes with a Dead Zone}

Like control schemes o and 6, schemes 2, 3 and 4 are coupled, but what sets them apart is that they include a dead zone in the middle of the screen. A dead zone is also called a "keyhole", and represents the area in which input will not be recognized from an input device until input goes above a specific threshold. For example, the player may have to move the mouse left or right a specified amount before the game will register mouse movement. What distinguishes these control schemes is the size and shape of the dead zones and how the input devices are controlled inside and outside of this zone. 


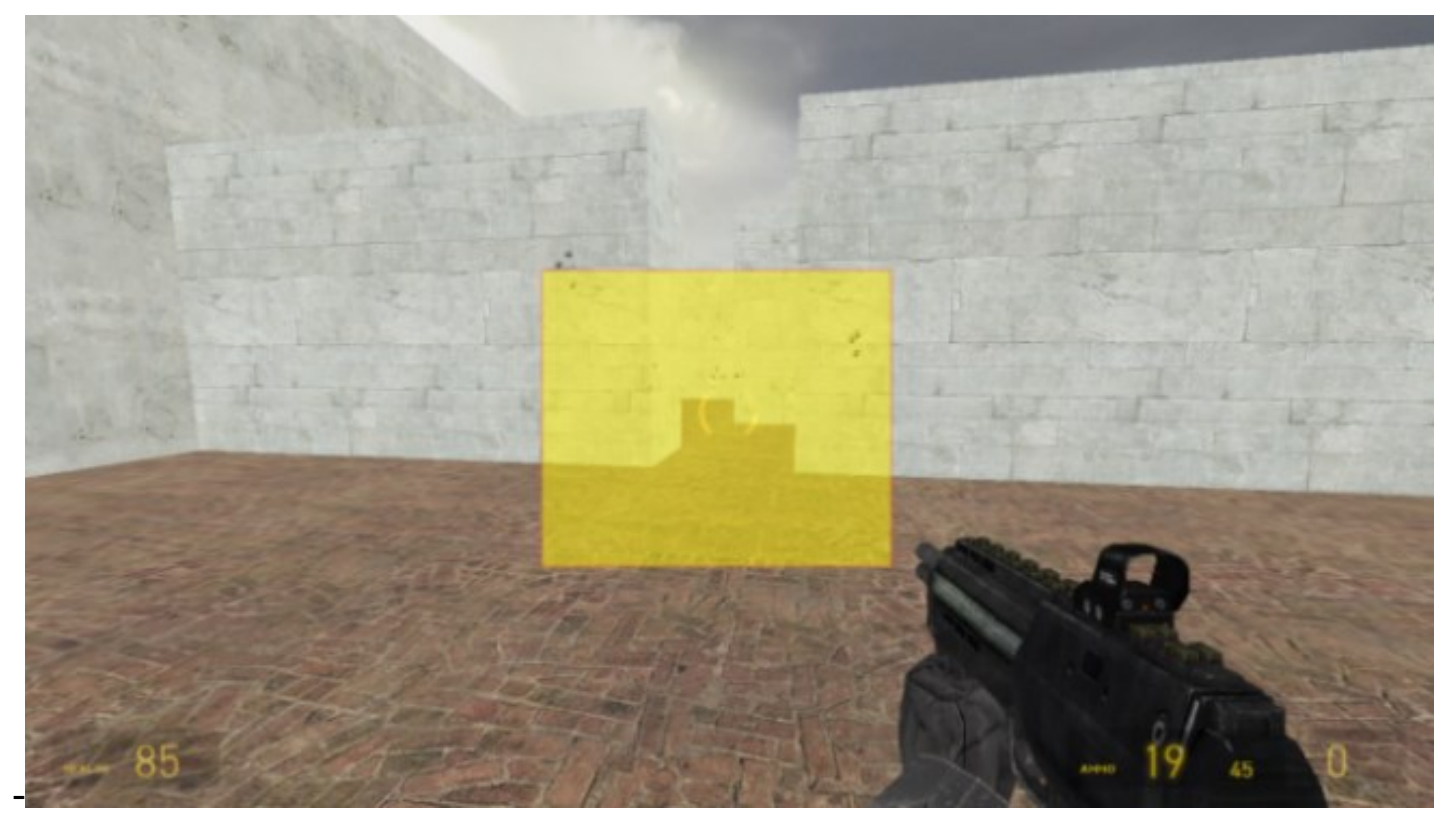

Illustration 7 Visualization of the dead zone for control scheme 2, study 1.

Control Scheme 2. In control scheme 2, the dead zone's bounding box is short and square (Illustration 7). Inside the dead zone, the mouse controls all functions: aiming, steering and camera view. The HMD aims and moves camera view without affecting steering so the player can use their head movement to make small adjustments to their aim. Outside of the dead zone, the HMD and the mouse are coupled to camera control, steering and aiming. Furthermore, the mouse z-axis input is disabled outside the dead zone so that only the HMD affects camera view. This makes it necessary to look up to select or shoot high targets. See Table 3. 


\begin{tabular}{|c|c|c|c|c|c|c|c|c|c|c|c|}
\hline \multicolumn{4}{|c|}{ Viewpoint Control } & \multicolumn{4}{|c|}{ Movement Direction } & \multicolumn{4}{|c|}{ Targeting } \\
\hline \multirow{2}{*}{$\begin{array}{l}\text { Input } \\
\text { Device }\end{array}$} & \multicolumn{3}{|c|}{ Control-Display Mappings } & \multirow{2}{*}{$\begin{array}{l}\text { Input } \\
\text { Device }\end{array}$} & \multicolumn{3}{|c|}{ Control-Display Mappings } & \multirow{2}{*}{$\begin{array}{l}\text { Input } \\
\text { Device }\end{array}$} & \multicolumn{3}{|c|}{ Control-Display Mappings } \\
\hline & Axis & Control & Display & & Axis & Control & Display & & Axis & Control & Display \\
\hline \multirow[t]{6}{*}{ Mouse } & $x$ & & & \multirow[t]{6}{*}{ Mouse } & $x$ & & & \multirow[t]{6}{*}{ Mouse } & $x$ & & \\
\hline & $y$ & & & & $y$ & & & & $y$ & & \\
\hline & $z$ & & & & $z$ & & & & $z$ & & \\
\hline & $\theta_{x}$ & & $\bullet$ & & $\theta_{x}$ & & & & $\theta_{x}$ & & • \\
\hline & $\theta_{y}$ & & b & & $\theta_{y}$ & & 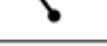 & & $\theta_{y}$ & & 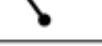 \\
\hline & $\theta_{z}$ & & & & $\theta_{z}$ & & & & $\theta_{z}$ & & \\
\hline \multirow[t]{6}{*}{ HMD } & $x$ & & $\longrightarrow$ & \multirow{6}{*}{$\begin{array}{l}\text { HMD } \\
\text { (outside } \\
\mathrm{Dz} \\
\text { only) }\end{array}$} & $x$ & & & \multirow[t]{6}{*}{ HMD } & $x$ & & $\longrightarrow$ \\
\hline & $y$ & & & & $y$ & & & & $y$ & & \\
\hline & $z$ & & $\longrightarrow$ & & $z$ & & & & $z$ & & $\longrightarrow$ \\
\hline & $\theta_{x}$ & & & & $\theta_{x}$ & & & & $\theta_{x}$ & & \\
\hline & $\theta_{y}$ & $\bullet$ & $\longrightarrow$ & & $\theta_{y}$ & $\bullet$ & $\longrightarrow$ & & $\theta_{y}$ & $\bullet$ & \\
\hline & $\theta_{z}$ & $\bullet$ & $\longrightarrow$ & & $\theta_{z}$ & & & & $\theta_{z}$ & $\bullet$ & \\
\hline
\end{tabular}

Table 3. Control-display mappings for control scheme 2.

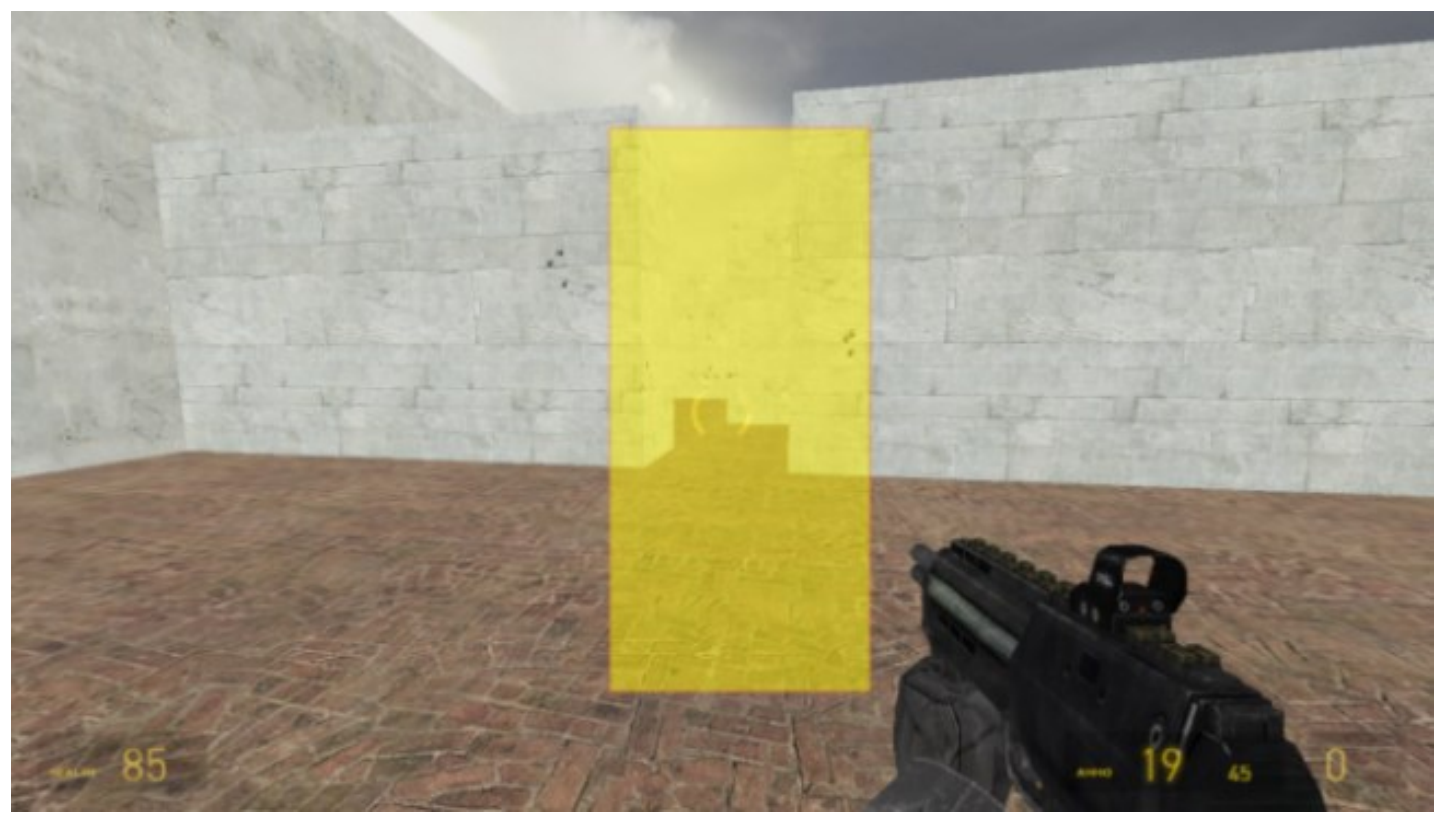

Illustration 8 Visualization of dead zone for control scheme 3 and 4, study 1.

Control Scheme 3. The dead zone for control scheme 3 is narrow and tall (Illustration 8). Inside the dead zone, the HMD controls camera view and steers, while the mouse controls targeting. This means that the target is not bound to the centre of the screen. Outside of the dead zone, both the mouse and HMD are coupled 
to camera control, steering and aiming and so either device can be used to support these functions. See Table 4 .

\begin{tabular}{|c|c|c|c|c|c|c|c|c|c|c|c|}
\hline \multicolumn{4}{|c|}{ Viewpoint Control } & \multicolumn{4}{|c|}{ Movement Direction } & \multicolumn{4}{|c|}{ Targeting } \\
\hline \multirow{2}{*}{$\begin{array}{l}\text { Input } \\
\text { Device }\end{array}$} & \multicolumn{3}{|c|}{ Control-Display Mappings } & \multirow{2}{*}{$\begin{array}{l}\text { Input } \\
\text { Device }\end{array}$} & \multicolumn{3}{|c|}{ Control-Display Mappings } & \multirow{2}{*}{$\begin{array}{l}\text { Input } \\
\text { Device }\end{array}$} & \multicolumn{3}{|c|}{ Control-Display Mappings } \\
\hline & Axis & Control & Display & & Axis & Control & Display & & Axis & Control & Display \\
\hline \multirow{6}{*}{$\begin{array}{l}\text { Mouse } \\
\text { (outside } \\
\text { DZ only) }\end{array}$} & $x$ & 9 & & \multirow{6}{*}{$\begin{array}{l}\text { Mouse } \\
\text { (outside } \\
\text { DZ only) }\end{array}$} & $x$ & & & \multirow[t]{6}{*}{ Mouse } & $x$ & & \\
\hline & $y$ & & & & $y$ & & & & $y$ & & \\
\hline & $z$ & & & & $z$ & & & & $z$ & & \\
\hline & $\theta_{x}$ & & & & $\theta_{x}$ & & & & $\theta_{x}$ & & $\bullet$ \\
\hline & $\theta_{y}$ & & $\bullet$ & & $\theta_{y}$ & & $b$ & & $\theta_{y}$ & & 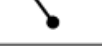 \\
\hline & $\theta_{z}$ & & & & $\theta_{z}$ & & & & $\theta_{z}$ & & \\
\hline \multirow[t]{6}{*}{ HMD } & $x$ & $\bullet$ & $\longrightarrow$ & \multirow[t]{6}{*}{ HMD } & $x$ & & & \multirow[t]{6}{*}{ HMD } & $x$ & $\bullet$ & $\longrightarrow$ \\
\hline & $y$ & $\bullet$ & $\longrightarrow$ & & $y$ & & & & $y$ & $\bullet$ & $\longrightarrow$ \\
\hline & $z$ & $\bullet$ & $\longrightarrow$ & & $z$ & & & & $z$ & $\bullet$ & $\longrightarrow$ \\
\hline & $\theta_{x}$ & & $\longrightarrow$ & & $\theta_{x}$ & & & & $\theta_{x}$ & & $\longrightarrow$ \\
\hline & $\theta_{y}$ & & $\longrightarrow$ & & $\theta_{y}$ & $\bullet$ & $\longrightarrow$ & & $\theta_{y}$ & $\bullet$ & $\longrightarrow$ \\
\hline & $\theta_{z}$ & $\bullet$ & $\longrightarrow$ & & $\theta_{z}$ & & & & $\theta_{z}$ & $\bullet$ & $\longrightarrow$ \\
\hline
\end{tabular}

Table 4. Control-display mappings for control scheme 3.

Control Scheme 4. Like control scheme 3, the dead zone for scheme 4 is narrow and tall (Illustration 8). Inside the dead zone, the mouse steers and aims but does not affect the camera view-instead, the HMD rotates the view relative to the avatar's movement direction. The targeting reticule is therefore not fixed to the center of the screen in this scheme. Outside of the dead zone, the mouse will steer, aim and also appear to affect the camera view, which will be dragged along with avatar movement direction rotation because of its relative orientation. See Table 5 . 


\begin{tabular}{|c|c|c|c|c|c|c|c|c|c|c|c|}
\hline \multicolumn{4}{|c|}{ Viewpoint Control } & \multicolumn{4}{|c|}{ Movement Direction } & \multicolumn{4}{|c|}{ Targeting } \\
\hline \multirow{2}{*}{$\begin{array}{l}\text { Input } \\
\text { Device }\end{array}$} & \multicolumn{3}{|c|}{ Control-Display Mappings } & \multirow{2}{*}{$\begin{array}{c}\text { Input } \\
\text { Device }\end{array}$} & \multicolumn{3}{|c|}{ Control-Display Mappings } & \multirow{2}{*}{$\begin{array}{c}\text { Input } \\
\text { Device }\end{array}$} & \multicolumn{3}{|c|}{ Control-Display Mappings } \\
\hline & Axis & Control & Display & & Axis & Control & Display & & Axis & Control & Display \\
\hline \multirow{6}{*}{$\begin{array}{l}\text { Mouse } \\
\text { (outside } \\
\text { DZ only) }\end{array}$} & $x$ & 9 & & \multirow[t]{6}{*}{ Mouse } & $x$ & & & \multirow[t]{6}{*}{ Mouse } & $x$ & & \\
\hline & $y$ & & & & $y$ & & & & $y$ & & \\
\hline & $z$ & & & & $z$ & & & & $z$ & $a$ & \\
\hline & $\theta_{x}$ & & & & $\theta_{x}$ & & & & $\theta_{x}$ & & $\bullet$ \\
\hline & $\theta_{y}$ & & $b$ & & $\theta_{y}$ & & $b$ & & $\theta_{y}$ & & 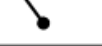 \\
\hline & $\theta_{z}$ & & & & $\theta_{z}$ & & & & $\theta_{z}$ & & \\
\hline \multirow{6}{*}{$\begin{array}{l}\text { HMD } \\
\text { (relative } \\
\text { to mov. } \\
\text { dir.) }\end{array}$} & $x$ & $\bullet$ & $\longrightarrow$ & & & & & & & & \\
\hline & $y$ & $\bullet$ & $\longrightarrow$ & & & & & & & & \\
\hline & $z$ & $\bullet$ & $\longrightarrow$ & & & & & & & & \\
\hline & $\theta_{x}$ & & $\longrightarrow$ & & & & & & & & \\
\hline & $\theta_{y}$ & & $\longrightarrow$ & & & & & & & & \\
\hline & $\theta_{z}$ & $\bullet$ & $\longrightarrow$ & & & & & & & & \\
\hline
\end{tabular}

Table 5. Control-display mappings for control scheme 4.

\subsubsection{Decoupled Control Scheme}

Unlike coupled control schemes (in which the rift and the mouse control targeting, steering and camera view), decoupled schemes assign different functions to the mouse and the HMD.

Control Scheme 1. In this scheme, the mouse controls the direction of movement, but the HMD controls the camera view. The targeting reticule is fixed in the center of the camera view. This scheme allows the player to look around the environment independent from the direction of avatar movement. Thus, the scheme is appropriate for driving and flying games because it mimics the experience of sitting in a cockpit and piloting a vessel where moving your head only allows one to look around, but not steer. See Table 6. 


\begin{tabular}{|c|c|c|c|c|c|c|c|c|c|c|c|}
\hline \multicolumn{4}{|c|}{ Viewpoint Control } & \multicolumn{4}{|c|}{ Movement Direction } & \multicolumn{4}{|c|}{ Targeting } \\
\hline \multirow{2}{*}{$\begin{array}{l}\text { Input } \\
\text { Device }\end{array}$} & \multicolumn{3}{|c|}{ Control-Display Mappings } & \multirow{2}{*}{$\begin{array}{l}\text { Input } \\
\text { Device }\end{array}$} & \multicolumn{3}{|c|}{ Control-Display Mappings } & \multirow{2}{*}{$\begin{array}{l}\text { Input } \\
\text { Device }\end{array}$} & \multicolumn{3}{|c|}{ Control-Display Mappings } \\
\hline & Axis & Control & Display & & Axis & Control & Display & & Axis & Control & Display \\
\hline \multirow[t]{6}{*}{ HMD } & $x$ & $\bullet$ & $\longrightarrow$ & \multirow[t]{6}{*}{ Mouse } & $x$ & & & \multirow[t]{6}{*}{ HMD } & $x$ & $\bullet$ & $\longrightarrow$ \\
\hline & $y$ & $\bullet$ & $\longrightarrow$ & & $y$ & & & & $y$ & $\bullet$ & $\longrightarrow$ \\
\hline & $z$ & $\bullet$ & $\longrightarrow$ & & $z$ & & & & $z$ & & $\longrightarrow$ \\
\hline & $\theta_{x}$ & 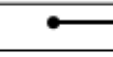 & $\longrightarrow$ & & $\theta_{x}$ & & & & $\theta_{x}$ & & $\longrightarrow$ \\
\hline & $\theta_{y}$ & $\bullet$ & $\longrightarrow$ & & $\theta_{y}$ & & $\checkmark$ & & $\theta_{y}$ & $\bullet$ & $\longrightarrow$ \\
\hline & $\theta_{z}$ & $\bullet$ & $\longrightarrow$ & & $\theta_{z}$ & & & & $\theta_{z}$ & $\bullet$ & $\longrightarrow$ \\
\hline
\end{tabular}

Table 6. Control-display mappings for control scheme 1.

\subsubsection{Task-based Game Maps}

As described in Chapter 2, the task can modify the effect a control scheme has on variables of interest. Because the primary tasks in FPS games are locomotion and targeting, we tested the control schemes for each of these tasks. To do so, we built two custom game maps with the Hammer map editor that is included in Valve Software's Source Software Developer Kit (SDK) [81]. These "maps" correspond to the virtual environments that players experienced in our study tasks, which we will now describe.

\subsubsection{Locomotion Map}

The locomotion task (Illustration 9 \& Illustration 10) requires the player to maneuver through a single-path maze that features many twists and turns, analogous to the locomotion tasks users perform in various games. Participants were timed as they ran through the maze from start to end point as quickly as possible (the end point was clearly indicated) while avoiding touching the walls. If the player touched a wall, the game subtracted 1 hit point from the player per each 1 second interval. 


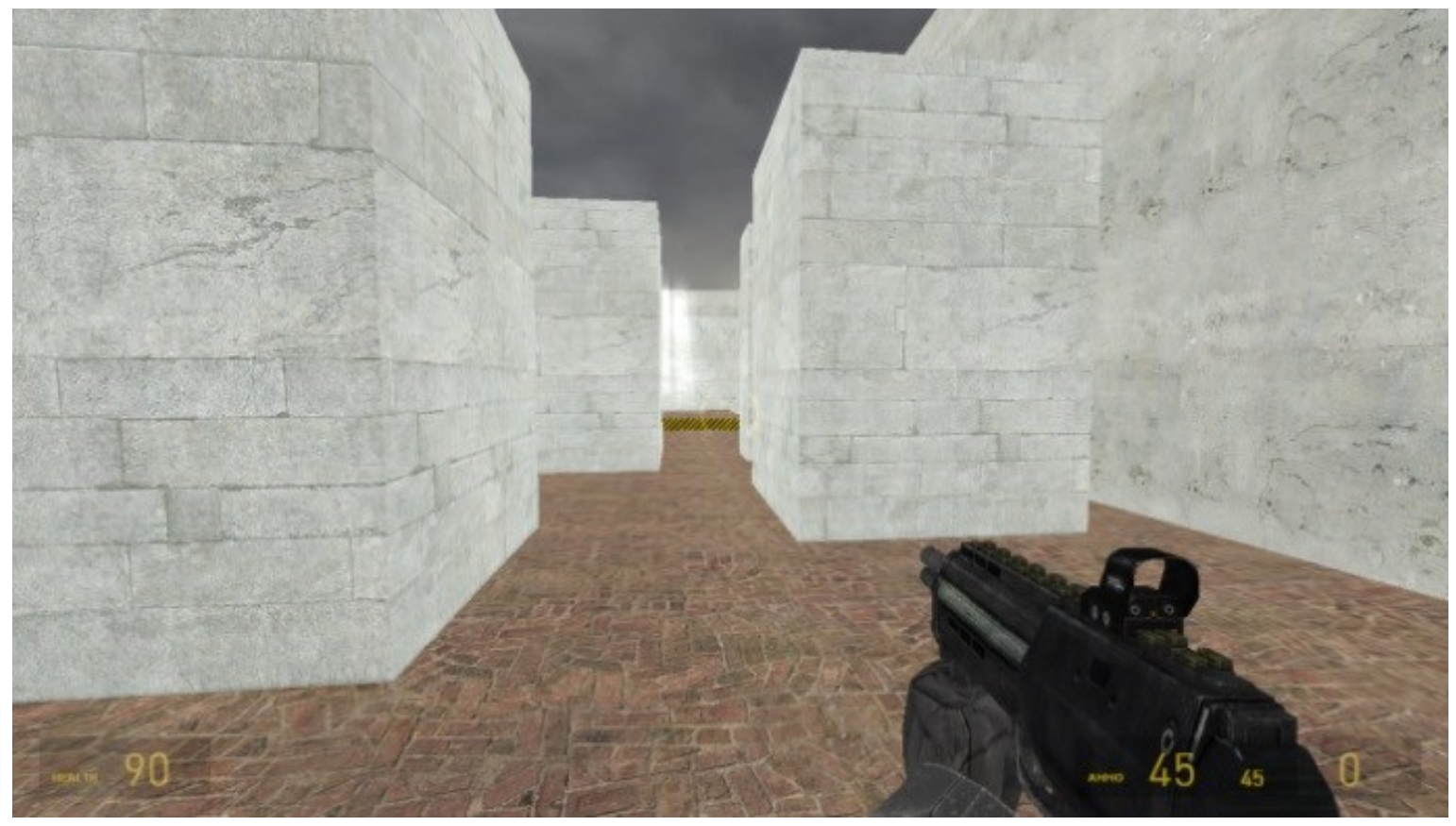

Illustration 9. In-game first person view of the locomotion map.

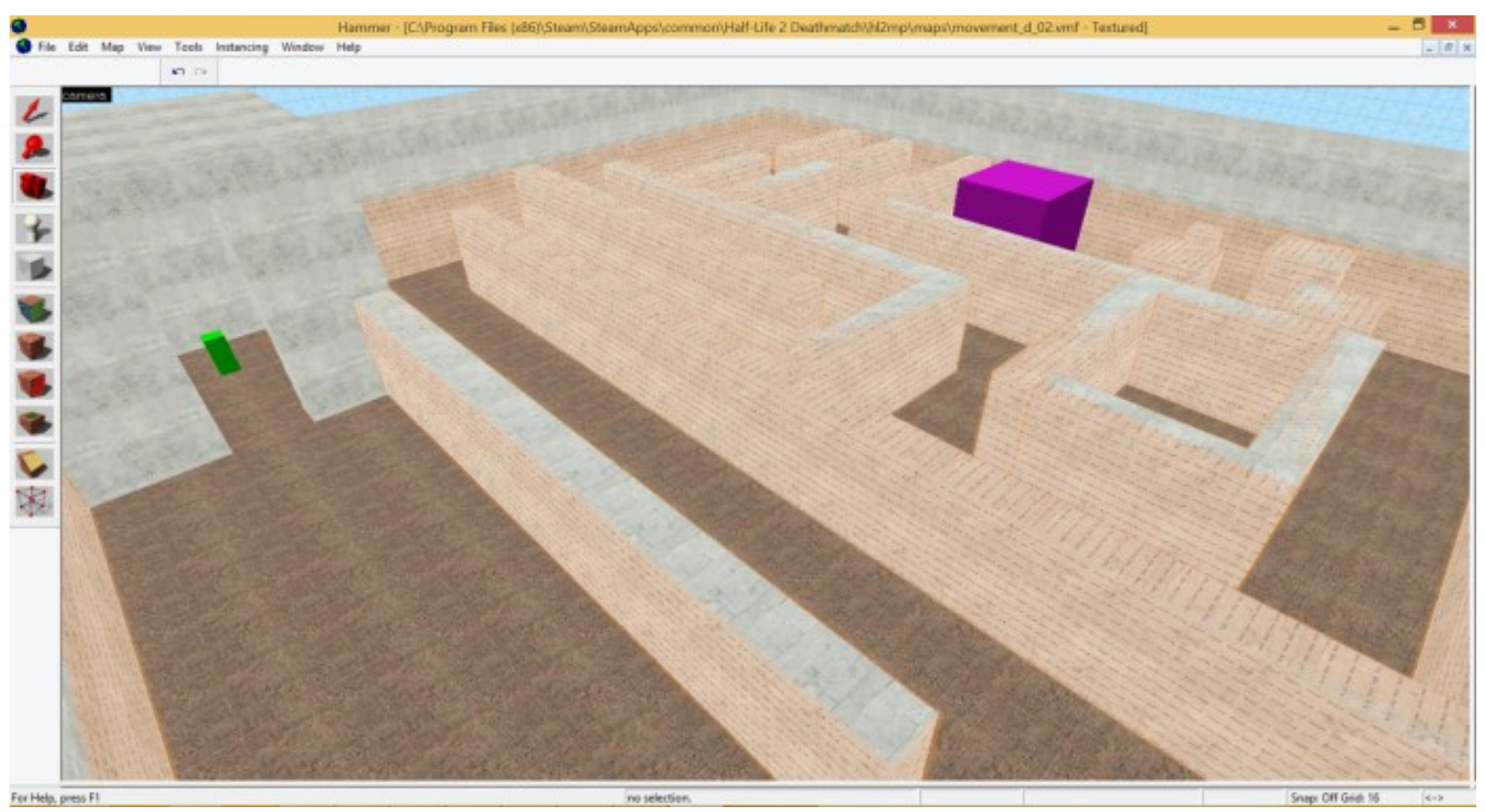

Illustration 10. Locomotion map in the Hammer map editor.

\subsubsection{Targeting Map}

The targeting task (Illustration 11 \& Illustration 12) requires the player to aim at and select targets (the usual application for this task in first-person games is shooting). 
The environment for this task presented the player with 14 exploding barrels and asked him or her to shoot as many barrels as possible within 60 seconds using a submachine gun with unlimited ammunition. We counted the number of barrels destroyed manually. To ensure constant availability of targets, these re-spawned after they exploded.

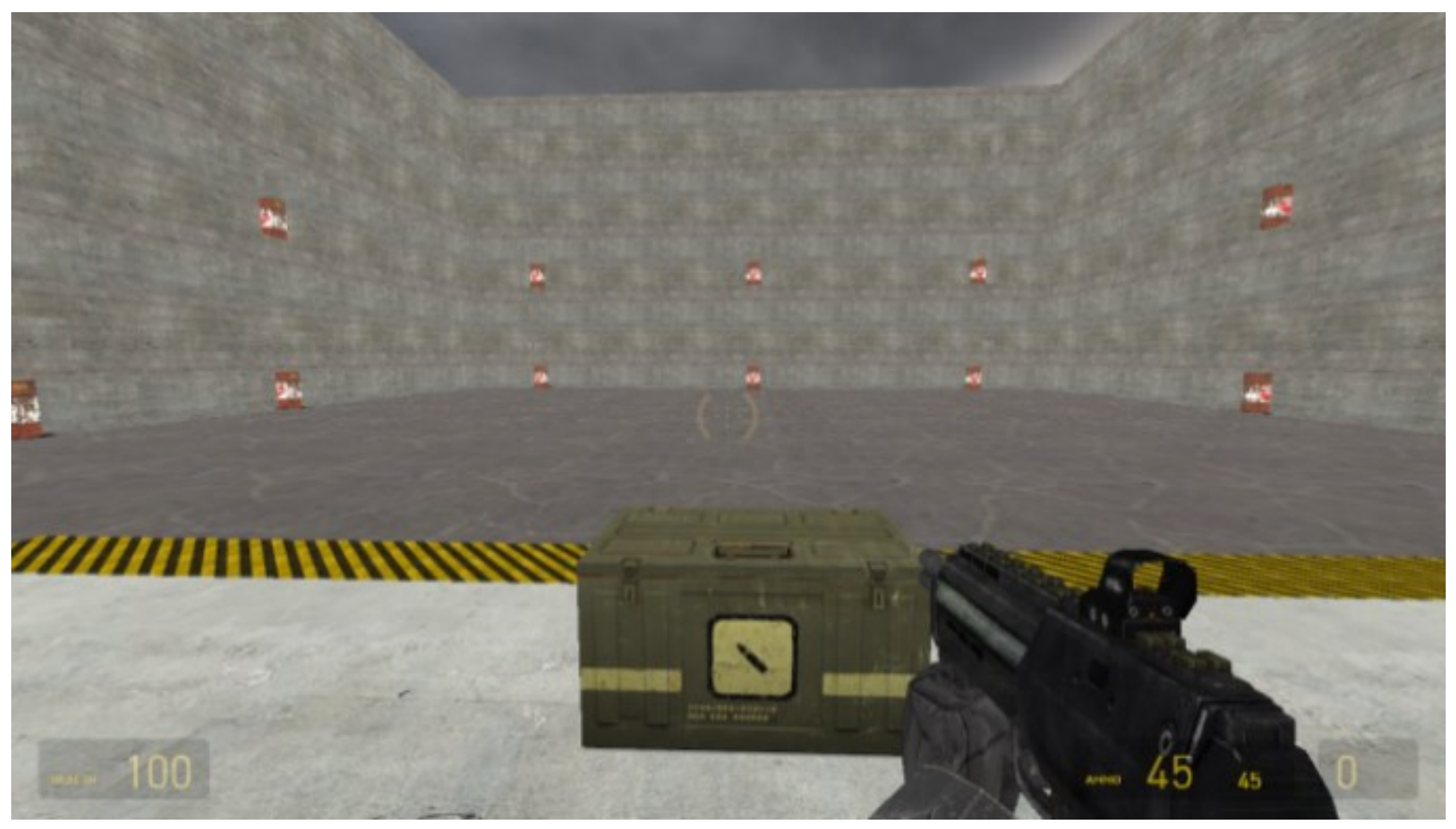

Illustration 11. In-game first-person view of the targeting map, study 1. 


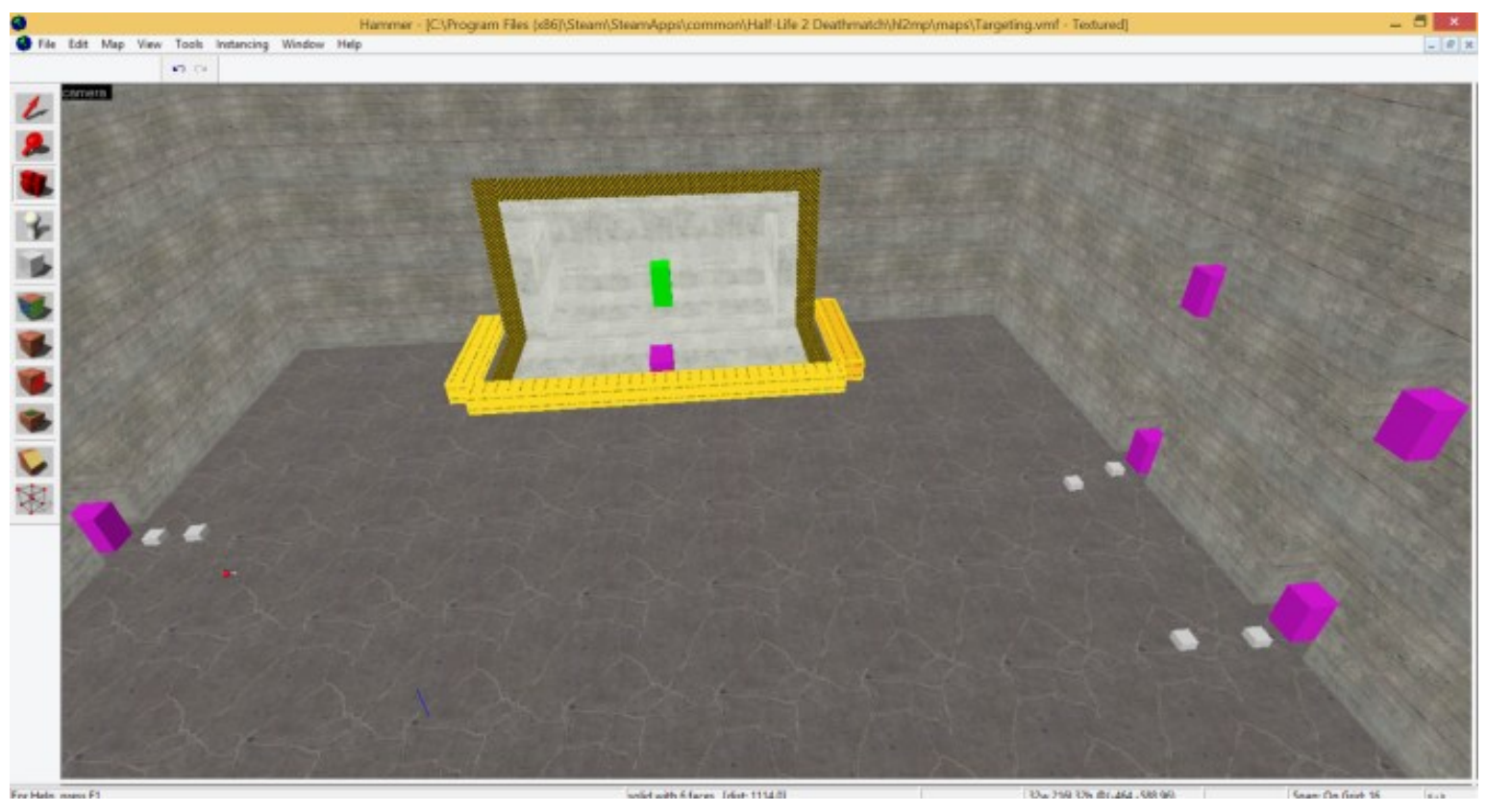

Illustration 12. Targeting map taken in the Hammer map editor, study 1.

\subsection{Measures}

To accurately assess how each control scheme affected participants, researchers collected both quantitative and qualitative data measuring usability and immersion constructs.

\subsubsection{Usability Measures}

As the goal of this study is to evaluate the usability of control schemes for VR FPS, to this end, we collected empirical data on usability metrics derived from International Organization for Standardization (ISO) definition: "The extent to which a product can be used by specified users to achieve specified goals with effectiveness, efficiency, and satisfaction in a specified context of use." [72] From this definition, we isolated the usability variables (see summary of usability metrics in Table 7): 
- Effectiveness: "The accuracy and completeness with which specified users can achieve specified goals in particular environments." [72]

- Efficiency: "The resources expended in relation to the accuracy and completeness of goals achieved." [72]

- Satisfaction: "The comfort and acceptability of the work system to its users and other people affected by its use."[72]

Since the task determines the relevance of a given measure, our usability measures were based on the type of task. For the locomotion task, we used two measures: (1) time, obtained by recording how long it took for the participant to traverse the environment and (2) errors, where an error occurred when the player's avatar touched the walls of the maze. For the targeting task, we used one measure, namely "kills", corresponding to the number of barrels that a player exploded. We also gathered qualitative data about the control schemes' usability via questionnaire and semi-structured interviews. 


\begin{tabular}{|c|c|c|c|c|}
\hline Data Type & Variable & Measures Used & Collection Method & Data Analysis \\
\hline \multirow{3}{*}{ QUAN } & Effectiveness & Performance scores & $\begin{array}{l}\text { Locomotion Task: Error rate } \\
\text { (Derived from number of } \\
\text { collisions with walls) } \\
\text { Targeting Task: Number of } \\
\text { "kills" or barrels destroyed. }\end{array}$ & 1-way ANOVA \\
\hline & Efficiency & Performance scores & $\begin{array}{l}\text { Locomotion Task: Time to } \\
\text { complete maze. }\end{array}$ & 1-way ANOVA \\
\hline & Satisfaction & $\begin{array}{l}\text { Control Scheme } \\
\text { Preference } \\
\text { Questionnaire }\end{array}$ & $\begin{array}{l}\text { Preference scores: Players } \\
\text { were asked to rate each control } \\
\text { scheme on a scale of } 1 \text { to } 5 \text {. }\end{array}$ & $\begin{array}{l}\text { Preference score: } \\
\text { Wilcoxon rank. }\end{array}$ \\
\hline QUAL & Satisfaction & $\begin{array}{l}\text { Semi-Structured } \\
\text { Interviews } \\
\text { and; } \\
\text { Control Scheme } \\
\text { Preference } \\
\text { Questionnaire }\end{array}$ & $\begin{array}{l}\text { Both questionnaires included } \\
\text { open-ended questions that } \\
\text { asked players to give feedback } \\
\text { about each control scheme and } \\
\text { explain the reasons for their } \\
\text { control scheme preference } \\
\text { rankings. }\end{array}$ & $\begin{array}{l}\text { Qualitative analysis: } \\
\text { Identify player } \\
\text { feedback relating to } \\
\text { satisfaction. }\end{array}$ \\
\hline
\end{tabular}

Table 7. Usability measures, collection method and analysis, study 1.

\subsubsection{Control Scheme Preference Questionnaire}

To identify the control scheme participants that preferred overall, we designed the Control Scheme Preference Questionnaire (Appendix C). The questionnaire asked participants to rate how much they liked each control scheme using a scale of 1 to 5 , with 1 being "not at all" and 5 being "very much so". Also, the questionnaire asked participants to rank the six VR control schemes in order of preference. After they completed the questionnaire, we asked participants to explain why they preferred their first-ranked control scheme and to describe any issues related to their least preferred control scheme.

\subsubsection{Semi-structured Interviews}

After completing both the targeting and locomotion tasks for a control scheme, we asked participants to comment on the usability of each control scheme ("What did you think of $x$ control scheme") using a retrospective think-aloud protocol. Follow-up 
questions were employed when the interviewer required clarification on the answer given. To end the interview and give participants a chance to bring up any other issues, the interviewer also asked: "Did you have any other thoughts about this control scheme?"

\subsubsection{Immersion Measures}

Satisfaction in productivity software is taken from meeting goals related to a specific task and context, whereas while playing games, satisfaction is taken from the process of using the software [23], or of being "immersed" in play. Because video games are different from software for productive work, it requires new constructs and measures to probe aspects of satisfaction. Immersion is a construct specific to videogames that attempts to define the experience of being engrossed in game playing. We will use the Immersive Experiences Questionnaire to measure immersion while using the different control schemes as well as identifying feedback about immersion in qualitative data. See Table 8 for a summary of immersion measures.

\begin{tabular}{|c|l|l|l|l|}
\hline Data Type & Variable & \multicolumn{1}{|c|}{ Measures Used } & \multicolumn{1}{c|}{ Collection Method } & Analysis \\
\hline QUAN & Immersion & $\begin{array}{l}\text { Immersive } \\
\text { Experiences } \\
\text { Questionnaire }\end{array}$ & Likert rating scale \\
\hline QUAL & Immersion & $\begin{array}{l}\text { Semi-structured } \\
\text { Interviews, } \\
\text { And; } \\
\text { Control Scheme } \\
\text { Preferences } \\
\text { Questionnaire }\end{array}$ & $\begin{array}{l}\text { Players were asked to give } \\
\text { feedback about each control } \\
\text { scheme and explain the } \\
\text { reasons for their control } \\
\text { scheme preferences. }\end{array}$ & $\begin{array}{l}\text { Qualitative analysis: } \\
\text { Identify player } \\
\text { feedback relating to } \\
\text { immersion. }\end{array}$ \\
\hline
\end{tabular}

Table 8. Immersion measures, collection method and analysis, study 1

\subsubsection{The Immersive Experiences Questionnaire}

In order to measure how immersed participants felt while using each control scheme, we administered the Immersive Experiences Questionnaire developed by Jennet and 
Cox [37]. The questionnaire measures four factors that contribute to immersion: cognitive involvement, emotional involvement, controls, and real-world dissociation. Our questionnaire included a subset of 31 questions taken from the original 33question set (questions 23 and 24 of the questionnaire were omitted because they were not relevant to the present study, see Appendix D). Players were also asked to answer one additional question: "How immersed did you feel?" which Jennet and Cox describe as a "single question measure of immersion" that can be used to verify the results of the full questionnaire.

\subsection{Design}

We now describe the study we conducted to test the impact of the various control schemes. In order to measure the effect of control scheme, we conducted an experiment with a $6 \times 2$ within-subjects design. The two independent variables were control scheme and task. The control scheme independent variable has six levels representing the six VR control schemes. The task independent variable has 2 levels: the targeting and locomotion tasks. Therefore, the experiment consisted of 12 trials. Dependent variables (time and errors) tested usability on the locomotion task, while the dependent variable for the targeting task was the number of "kills".

To counteract any fatigue or practice effects, we used partial counterbalancing via a Latin square design, where a given participant experienced the control schemes in one of six possible orders. Table 9 shows the six possible orderings for the control schemes. We also alternated the order in which we presented the two tasks. 


\begin{tabular}{|c|c|c|c|c|c|c|}
\hline Order set & \multicolumn{7}{|c|}{ Control Schemes } \\
\hline $\mathbf{1}$ & 0 & 1 & 6 & 2 & 4 & 3 \\
\hline $\mathbf{2}$ & 1 & 2 & 0 & 3 & 6 & 4 \\
\hline $\mathbf{3}$ & 2 & 3 & 1 & 4 & 0 & 6 \\
\hline $\mathbf{4}$ & 3 & 4 & 2 & 6 & 1 & 0 \\
\hline $\mathbf{5}$ & 4 & 6 & 3 & 0 & 2 & 1 \\
\hline $\mathbf{6}$ & 6 & 0 & 4 & 1 & 3 & 2 \\
\hline
\end{tabular}

Table 9. Counterbalancing schedule, study 1.

\subsubsection{Mixed Methods}

To get a holistic view of the usability of the control schemes, we chose a mixed methods approach in which we collected and analyzed both qualitative and quantitative data. Specifically, we used convergent parallel mixed-methods [23] (see process flow diagram in Illustration 13) where all data was collected during the same study and qualitative and quantitative data were analysed separately before merging the results in the Discussion section. 


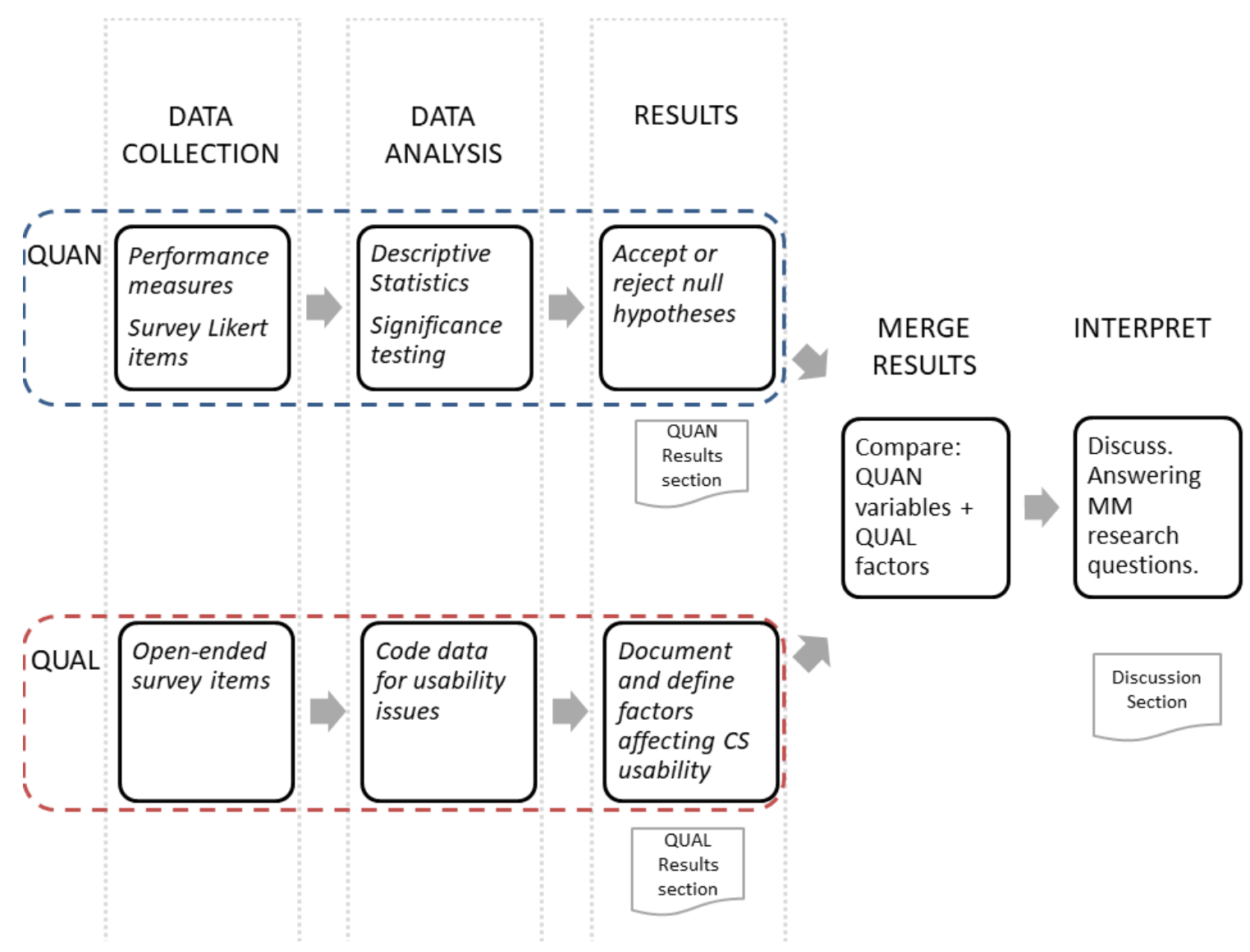

Illustration 13 Process flow for convergent parallel mixed-methods.

The convergent parallel typology is a subtype of triangulation designs that Creswell [24] recommends when "a researcher wants to directly compare and contrast quantitative statistical results with qualitative findings or to validate or expand quantitative results with qualitative data." To guide our analysis when merging and interpreting the qualitative and quantitative results, we added a set of mixed methods research questions. [75]:

1. Explanatory: Do the usability issues mentioned by participants help explain the effect of control schemes? 
2. Comparative: Are there points of convergence and divergence between the quantitative and qualitative data? What are the implications of the convergence and/or divergence?

3. Generative: Can we identify possible theories about the effect of the control schemes?

4. Generative: Can we make specific control scheme recommendations?

\subsection{Procedure}

Sessions were conducted individually in a private room within a research lab. The room was equipped with a computer that had keyboard, mouse, and Oculus Rift DK2 peripheral. After giving informed consent and receiving information on cybersickness, each participant was fitted with the Oculus Rift. Prior to starting the experimental tasks, participants were given a chance to acclimatize to VR by playing the DM_Overwatch level of $H L 2: D M$ (this took 2 - 5 minutes). During this acclimatization phase, the researcher asked the participants about their level of cybersickness (at this point in the study, two of the participants decided to drop out of the session due to cybersickness). Next, participants filled out the game experience questionnaire.

To begin the experiment, each participant completed the targeting and locomotion tasks for the first control scheme (the task order was alternated). After both tasks were completed for that control scheme, the researcher conducted a retrospective talk-aloud interview to gather feedback about the control scheme, and then asked the participant to complete the Control Scheme Preference Questionnaire and 
Immersive Experiences Questionnaire for that control scheme. This procedure was repeated for the remaining five control schemes (for a total of 12 trials), except that participants were asked to additionally rank the control schemes by preference as they experienced them. The study session took from 60 to 90 minutes to complete. Participants were given 10 dollars as compensation for their time.

\subsection{Results}

We begin with the quantitative results related to usability, immersion, and control scheme preference, and then present the qualitative analysis of the survey and interview data.

Recall that our study included six control schemes and a within-subject design. Our original analysis thus included all six schemes as a six level factor in a series of one way within-subjects ANOVAs for each dependent variable. However, we did not find significant differences between control schemes within a given scheme category (decoupled only had one scheme). For example, there were no significant differences between the coupled control schemes o and 6 for any of the dependent variables. Thus, to clarify the presentation of the results, we collapsed the data for the schemes in a given scheme category by aggregating for each dependent variable the data as follows: (1) the data from schemes o and 6, resulting in a coupled scheme variable; (2) from schemes 2, 3 and 4, resulting in a dead zone scheme variable. The third variable of interest pertains to the decoupled scheme variable (comprised of data from scheme 1). We acknowledge that the Latin square algorithm we used is designed for six rather than three conditions-this could pose a problem if the conclusions reached were different for the collapsed data (three conditions) vs. 
original data (six conditions). However, the results are analogous for the two sets of analyses in terms of the conclusions drawn for all overall effects. For the sake of completeness, the analysis that included each of the six schemes is provided in Appendix A.

\subsubsection{Usability Results}

To investigate the effect of each control scheme type (coupled, dead zone, decoupled) on control scheme usability, we ran one-way, within-subjects ANOVAs for each dependent variable. Control scheme type was the independent variable in the ANOVA model. Recall that the locomotion task had two dependent variables, namely total errors and time, and the targeting task had one dependent variable, namely number of kills.

\subsubsection{Locomotion task: Errors}

We begin with the descriptive statistics for total errors. As shown in Illustration 14, the coupled control scheme type resulted in the fewest number of errors, while the decoupled control scheme type was associated with the highest number of errors.

This interpretation was confirmed by the ANOVA, which indicated a significant main effect of control scheme type on mean number of errors, $F(1.16,40.68)=10.92, p=$ $\left..001, \boldsymbol{\eta}_{\mathrm{p}}{ }^{2}=.238\right)$. Mauchley's test of sphericity was significant for all ANOVAs and so we followed the standard convention of reporting test statistics using GreenhouseGeisser values. Pairwise comparisons with Bonferroni adjustment to adjust for familywise error indicated that the coupled control schemes resulted in significantly fewer errors than the decoupled control schemes $(p=.001)$ than the dead zone 
control schemes $(p=.006)$, while the decoupled control schemes resulted in significantly more errors than the dead zone control schemes $(p=.034)$.
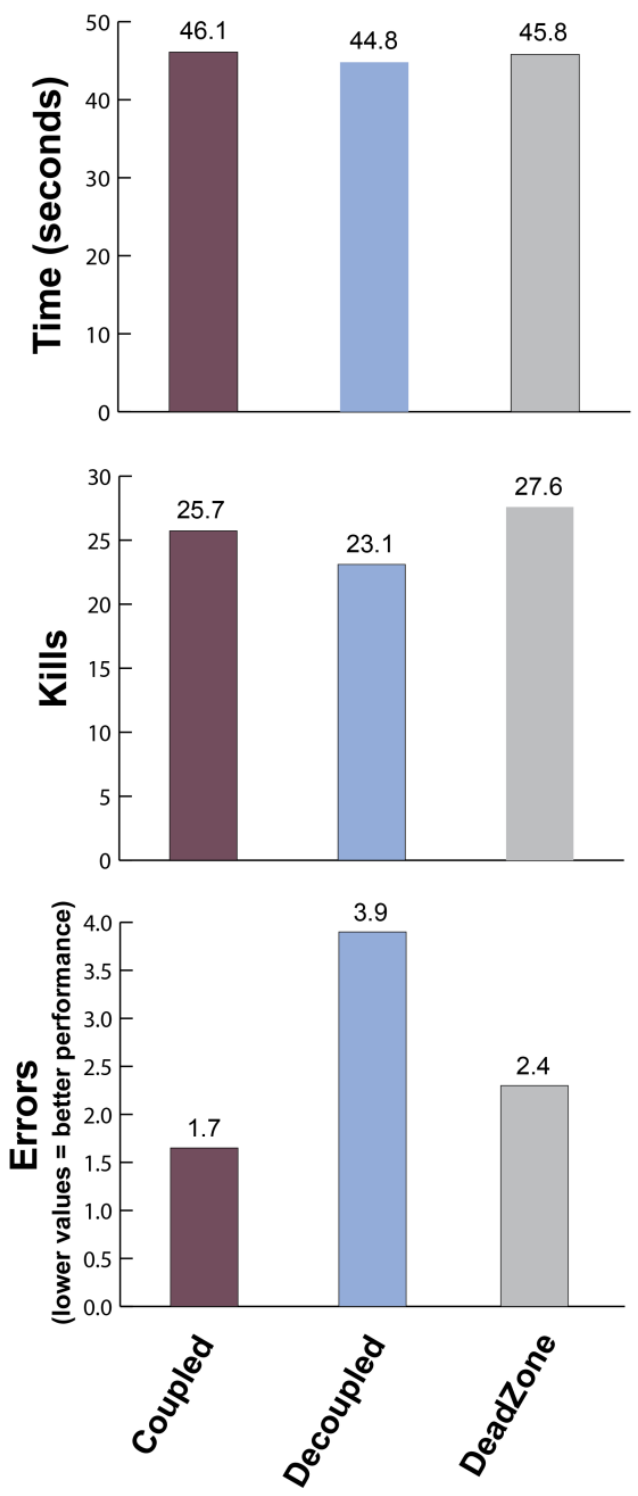

Illustration 14 Usability results: mean errors (bottom); mean kills (middle); and mean time (top), by control scheme type, study 1.

\subsubsection{Locomotion Task: Time}

Illustration 14 shows that the mean time spent performing the locomotion task for the various control scheme types was very similar. The ANOVA confirmed that control scheme type did not have a significant main effect on amount of time spent, $F(2,70)=$ $.11, p<.893, \boldsymbol{\eta}_{\mathrm{p}}^{2}=.003$. Thus, we did not find evidence that control scheme type influenced the time needed to execute the locomotion task.

\subsubsection{Targeting Task: Kills}

As shown in Illustration 14 , the mean number of kills was lowest for the decoupled control scheme and highest for the dead zone control scheme. The ANOVA confirmed that there was a significant main effect of control scheme type on the number of kills, $F(2,70)=27.36, p<.001, \boldsymbol{\eta}_{\mathrm{p}^{2}}=$ .439. Pairwise comparisons with Bonferroni adjustment found that the dead zone control schemes had significantly more kills than the coupled control schemes ( $p=$ 
.002) and the decoupled control schemes $(p=.008)$, while the decoupled control schemes had significantly fewer overall kills than the dead zone control schemes $(p<$ .001). Thus, the dead zone schemes resulted in highest number of kills overall.
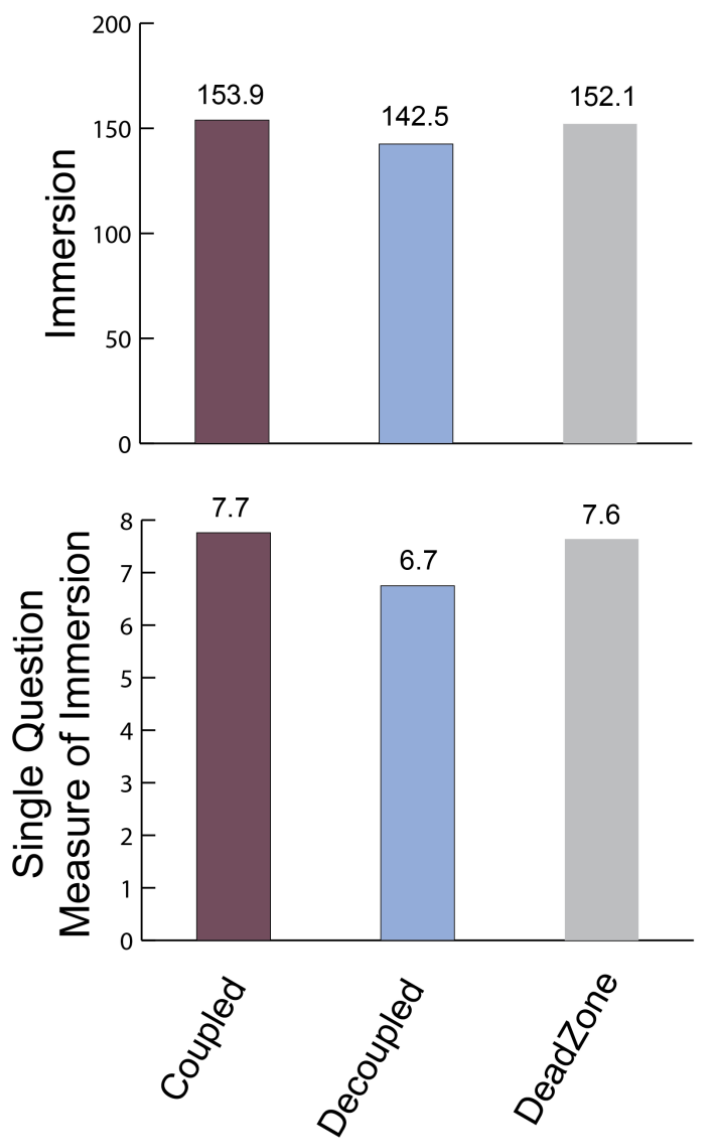

Illustration 15. Mean immersion scores (top) and mean scores for the single question measure of immersion (bottom), by control scheme type, study 1 ..

\subsubsection{Immersion Results}

Participants filled out the Immersive Experiences Questionnaire (IEQ) for each control scheme, producing six immersion scores per participant, one for each control scheme (note that participant's immersion scores were obtained by summing the ratings for all questions in their IEQ). We calculated the mean of the immersion scores per control scheme type. As the descriptive data in Illustration 15 shows, participants rated the decoupled control type as the least immersive.

A one-way repeated-measures ANOVA confirmed that there was a significant main effect of control scheme on immersion scores, $F(1.37,48.17)=7.43, p=.004, \boldsymbol{\eta}_{\mathrm{p}}{ }^{2}=.175$. Pairwise comparisons with Bonferroni adjustment indicated that immersion scores for the decoupled control scheme type were significantly lower than for the coupled control scheme type ( $p=$ 
.01) and the dead zone control scheme type $(p=.045)$. All other comparisons were not significant.

The above result that the decoupled scheme resulted in lowest immersion was mirrored in the confirmatory analysis we ran where we analyzed the scores for the single question measuring overall immersion (i.e., "How immersed did you feel?"): Coupled control scheme type was rated the most immersive $(M=7.76)$, with the dead zone control scheme type coming close behind $(M=7.63)$. The decoupled control scheme type was yet again rated the least immersive $(M=6.75)$ as shown in Illustration 15. A one-way repeated-measures ANOVA, confirmed that there was a significant main effect of immersion as measured by the single question measure of immersion, $F(1.43,50.11)=12.0, p<.001, \boldsymbol{\eta}_{\mathrm{p}}^{2}=.255$. Pairwise comparisons with Bonferroni adjustment indicated that immersion scores for the decoupled scheme type were significantly lower than for the coupled $(p<.001)$ and the dead zone control scheme type ( $p=.005)$. All other comparisons were not significant.

\subsubsection{Preference Results}

To analyze which control scheme players preferred, we analyzed the control scheme ratings from the Control Scheme Preference Questionnaire to get a control scheme rating for each control scheme type. As shown in Illustration 16, players preferred the coupled control schemes closely followed by the dead zone control schemes; decoupled control schemes were ranked lowest by a wide margin. 


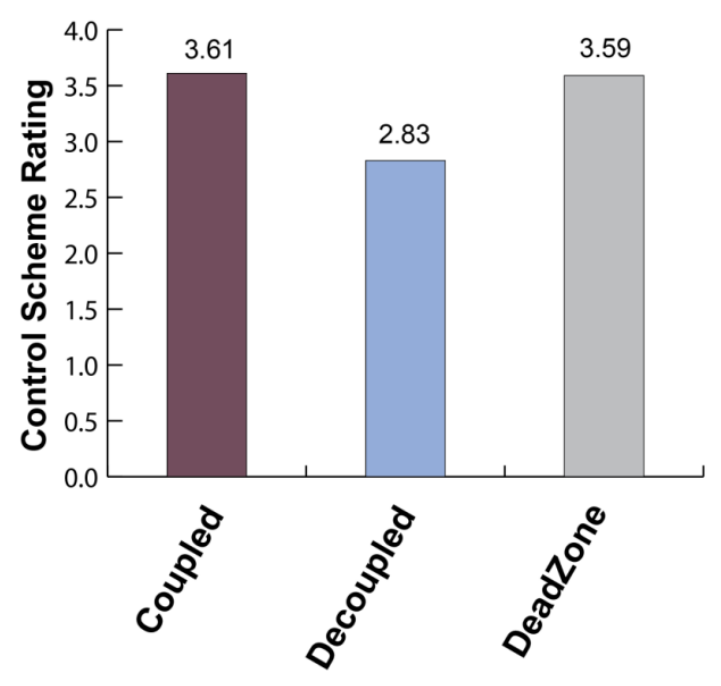

Illustration 16. Mean preference scores by control scheme type, study 1 .
A one-way repeated-measures ANOVA indicated a significant main effect of control scheme on player preference, $F(1.58,55.43)=6.01, p=.008, \eta_{p^{2}}=.147$. Pairwise comparisons with Bonferroni adjustment indicated that the decoupled control scheme type was rated significantly lower than both the coupled $(p=.017)$ and dead zone $(p=.052)$ control scheme types. All other comparisons were not significant.

\subsection{Qualitative Analysis}

To supplement the quantitative results, we analyzed qualitative data on the participant's experiences with each control scheme. To do so, we compiled transcripts of the retrospective talk-aloud sessions and the answers given in thee Control Scheme Preference Questionnaire, and after reading through the transcripts, we iteratively developed a set of codes that identified any usability issues or advantages for each control scheme. The data sets for each control scheme were coded using Atlas.ti [53] qualitative data analysis software. Our goals for the qualitative analysis were to obtain understanding of what features players appreciate (or not) in a VR control scheme. 


\subsubsection{Coupled Controls Scheme Impressions}

Recall that in coupled control schemes, the HMD and mouse controls the camera and steering the avatar simultaneously, i.e., the controls are coupled. There were two coupled control schemes in the study: control scheme o and control scheme 6 .

In general, participants that liked coupled schemes found them intuitive and easy to control for the locomotion task ( $\left.\mathrm{P}_{5}, \mathrm{P}_{13}, \mathrm{P}_{23}, \mathrm{P}_{26}, \mathrm{P}_{27}, \mathrm{P}_{33}, \mathrm{P}_{36}\right)$ because of how similar they are to traditional schemes. P36 said: "I felt it was closest to experience I was used to for FPS games." P5 said that this scheme "had a more comfortable transition between standard keyboard/mouse controls and VR".

Some participants found it frustrating that the mouse movement was constrained on the y-axis during the targeting task (P11, P15, P29). This was the only aspect of the controls that were not coupled, as the players had to use the HMD to move the camera (and the targeting reticule for control o) up and down-this was most noticeable during the targeting task for control scheme o. As participant 11 said, "having to crane my head up and down to aim vertically was uncomfortable and kind of annoying." Several participants found aiming with their head inefficient (P15, P19) and inaccurate ( $\left.\mathrm{P}_{33}, \mathrm{P}_{35}\right)$. On the other hand, some participants enjoyed having to aim with their heads because it allowed them to control the game with their body movements (P12, P30, P26, P34). P26 said: "it meant that I was very involved in the kinetic aspects of the game (i.e., turning my head), which definitely added a sense of realism to the gameplay." 


\subsubsection{Decoupled Control Scheme Impressions}

The decoupled control scheme type in this study was control scheme 1. In this control scheme, the player can look around the scene without affecting avatar steering.

The decoupled control scheme type was quite different from controls that participants were used to. The novelty of the controls was a negative for some participants ( $\left.\mathrm{P}_{5}, \mathrm{P}_{10}, \mathrm{P}_{27}, \mathrm{P}_{29}, \mathrm{P}_{33}\right)$. For example, $\mathrm{P}_{5}$ said that "it felt different enough from the standard keyboard/mouse controls that it felt a little alien." The novelty of the decoupled controls made them difficult for many participants to understand (P3, P6, P8, P10, P27, P29, P33). Many participants expected that their avatar would move in the direction of the camera, but since the controls were decoupled, the mouse modified the movement direction instead of the HMD. P8 expressed this by stating: "the angles while running would be off, where I would need to hold down W and D to move straight, and moving the mouse wouldn't help much." Even participants who figured out that the controls were decoupled found the interaction uncomfortable. P1o indicated that "I didn't like how part of the control was given over to where I was looking, especially during the shooting scenario. There was definitely more of a disconnect between where I wanted to move or what I wanted to do and the control." One strategy for navigation was to keep the head completely still and use only the mouse, but this was not well received. For instance, P36 said "I did not like this controls because I could not turn using my head. I had to keep my head unnaturally still in order to be able to turn, otherwise I would be confused about how much I had to turn my mouse to get the best turn radius.” 
The control scheme also presented challenges to four participants during the targeting task ( $\left.\mathrm{P}_{9}, \mathrm{P}_{4}, \mathrm{P} 6, \mathrm{P}_{3} 6\right)$. With this scheme, players needed to aim at targets with the HMD, an interaction that they deemed uncomfortable, inaccurate or inefficient. For instance, P4 wrote that "I didn't enjoy aiming with my head. It was inaccurate and nauseating.”

On the other hand, the control scheme's novelty as compared to mouselook was seen as a positive by several participants, lending it a feeling of realism because the HMD controlled the camera (P2, P35, P31, P34). For example, P2 indicated that "I think schemes where the mouse handles most or all of the aiming feels most natural, with the head having more control over the camera - this way is more relevant to real life in which what you see depends on your head direction and what you shoot depends on your hand position."; similarly, P35 shared that "it feels closer to walking in the real world." Other participants enjoyed the VR-ness of the scheme. One reason the scheme seemed so VR-specific was the ability to use head tracking meaningfully (P34, P35, P25): "Control schemes that barely used the Oculus made me think the game didn't even need it.”

Several participants found the control scheme immersive. Some simply enjoyed the decoupled nature of the control scheme (P25, P20). Specifically, the fact that where you looked was where the camera pointed and the mouse did not control the camera. In this way, $\mathrm{P} 4$ found that "There's no disconnect between the mouse and eye movement..." For some, it was the sensation of targeting with head movement that felt immersive (P31, P21). P21 wrote that "this scheme required me to move my head up and down to move the camera, rather than use the mouse. This felt immersive as I had no other option to see up/down, which would be the case in reality." 


\subsubsection{Dead Zone Control Scheme Impressions}

Dead zone control schemes all feature a dead zone, or "keyhole" in the middle of the screen in which input devices may not be recognized. There are three such control schemes in this study: 2, 3 and 4.

The most common feedback about the dead zone control schemes was that they were intuitive (P2, $\mathrm{P} 3, \mathrm{P} 4, \mathrm{P} 6, \mathrm{P} 10, \mathrm{P} 11, \mathrm{P} 18)$; possibly due to how similar the schemes were to mouselook. P4 said that "I felt like I was in control and it was a closer mapping from traditional control schemes." P2 said that "it felt like I was playing a regular first-person shooter." P19 said "it's familiar to what we are used to so far." Another common comment was that the dead zone schemes made it easy to move during the locomotion task (P15, P22, P30). Two participants felt that control scheme 3 was the most comfortable of all of the schemes and did not cause cybersickness (P30, P33). Interestingly, several participants found this familiarity was a limitation (P29, P31, P34, P35) because as P35 put it, "it felt the least like virtual reality."

The size and shape of the dead zone greatly influenced the gaming experience, and so here we breakdown the dead zone category to highlight aspects of its individual schemes that our participants mentioned. For control schemes 3 and 4, the dead zone was narrow and tall, which made the $\mathrm{x}$-axis more responsive and the $\mathrm{y}$-axis suitable for aiming. P14 summarized his impression of this by writing "I preferred the y-axis being "loose" as in having a set of bounds in which the mouse may move without affecting the camera. This lead to a superior targeting ability, as it was far easier to aim comfortably. While control scheme 6 had this, the bounds felt too large 
before the camera was affected and just didn't feel right. In regards to the $\mathrm{x}$-axis, I felt like having the "tight" controls (i.e., no bounds/direct camera control) was easier to use as there was no lag between turning with the mouse and turning while walking.”

On the other hand, there were several complaints about the dead zone for control scheme 2, which was shallow and wide. During the locomotion task, participants found the dead zone too wide, which made the mouse feel unresponsive to many participants (P2, P25, P14, P16). P14 said that "it always felt like there was a lag in its response due to having to reach the end of the bounds of the x-axis before the turn would commence." This seems to contradict the positive comments about the control scheme working well for locomotion. During the targeting task, several participants thought the dead zone was too shallow, which required them to use the HMD to move the reticule beyond the dead zone-for example, to the top of the screen (P25, P29, P33, P34). This meant that in certain situations the participants needed to use both input devices to target. Four participants noted that this made aiming at targets tiresome (P25, P29, P33, P34). For instance, P34 indicated that “I don't like to use both mouse and rift to shoot targets up high."

Control schemes 3 and 4 featured "free aim", where the targeting reticule was not fixed to the centre of the camera view, and could be moved using the mouse within the dead zone. Several participants liked this feature because they could move the target independent of the camera view (P6, P9, $\left.\mathrm{P}_{32}\right)$. $\mathrm{P}_{32}$ said that it was "easy to move the reticule around and it did not impede my movement...” 


\subsection{Discussion}

We compared three control scheme types in a VR game setting using both quantitative and qualitative methods. Since there isn't prior work on the impact of control schemes in VR games, we make a number of novel contributions.

First, our results indicate that of the two most common VR control schemes types (coupled and decoupled), the coupled control scheme results in better usability scores (errors and kills), immersion scores and player preference ratings than the decoupled scheme. The one exception is that the control scheme did not have a significant main effect on the time measure. Participants maintained a consistent speed regardless of the control scheme, the impact of which was that they would incur more or less errors. This finding suggests that players do not choose slow down and be more cautious in response to control schemes that offer less precise control.

Feedback given by participants for the coupled control scheme also indicates that it provides the best player experience overall, while the decoupled scheme was related to a more negative player experience. We hypothesized that the coupled control schemes would be more intuitive and have a positive effect on usability scores, based on the only prior research (albeit research using older and seldom-used input devices and not in a gaming context, or using experienced gamers as participants) [10, 65]. However, our expectation that the decoupled scheme would be more immersive because of its use of head-tracking rather than mouse and keyboard input was not confirmed (although a minority of participants did express preference for the decoupled scheme and their reasons were related to immersion). 
Second, our study contained a qualitative component based on participant feedback on their experience playing the game. We isolated four main usability factors in the player experience data across the control schemes that influenced whether players liked or disliked a control scheme type. These usability factors allow us to interpret the performance data based on the reasoning given by the participants. More importantly, it gives us insight into what players like and dislike about a control schemes and provided best practices for designing control schemes. These factors can be of use to game designers when creating novel control schemes or choosing a control scheme. The four usability factors we identified and their implications are as follows:

1. Whether the control scheme was coupled or decoupled.

2. How familiar the control scheme was, compared to mouselook.

3. Which input device was mapped to a display property.

4. The size and shape of the dead zone.

We discuss each of these factors below, integrating our qualitative and quantitative results where appropriate.

Coupled vs. decoupled. Our analysis suggests that overall, the most important factor in terms of the effect on preference and usability scores was whether the control scheme was coupled or decoupled. Most participants found the coupled schemes "intuitive" and "easy", and the quantitative measures reflect this (usability, immersion, and preference scores are highest for coupled control schemes). In contrast, the decoupled control scheme was the only scheme in the study where the 
camera and steering were decoupled. Fourteen participants rated this control scheme their least favourite, many reporting that they found it difficult to control the camera and steering separately, and this was reflected in the usability scores. In general, the majority of users were too frustrated by the decoupled control scheme to appreciate using the head-tracking input that is prominent for this scheme. However, six participants (16.7\%) preferred the decoupled control scheme over the other schemes. The reasons given for their preference centre on the scheme's realism and how it used the head mounted display input more than other schemes. In fact, several of these participants were disappointed by the coupled schemes because they felt that just using the mouse was less immersive and did not take advantage of the head mounted display as an input device. Perhaps these users were more flexible and able to adjust quickly to the novel experience of using head-tracking as game input.

Familiarity. The familiarity of the control scheme was also a factor driving the results. This is consistent with prior research (for desktop 3D games), which has shown that players prefer familiar controls and controllers [23, 31, 40,67]. We expected that the prior gaming experience of the participants would affect how intuitive a control scheme felt, which is why we chose to study experienced gamers. This factor is related to whether the schemes were coupled and decoupled. The decoupled scheme deviated from the mouselook control scheme that most participants are familiar with and had the lowest scores for all measures. The coupled schemes, including ones with the dead zone, were more favourably regarded. Feedback for these schemes said that they were easy to use by virtue of being familiar, and the usability scores reflect this finding. 
Input device. Another important factor was which device (HMD or mouse) controlled game functions. Perhaps again due to familiarity, most participants preferred to use the mouse to control all functions (targeting, movement direction, and camera view). As far as targeting, the decoupled control schemes forced players to aim using their heads in order to shoot targets during the targeting task, which many participants found fatiguing and less accurate than using the mouse to aim. One coupled control scheme (control scheme o) was also criticised for this. Usability scores for the targeting task bear out the feedback given by participants: control schemes that used the head mounted display for targeting had the lowest usability scores for the targeting task and control schemes that used the mouse to aim had the highest usability scores. As far as the other two functions (camera view and movement direction), participants also preferred to use mouse input. People used adjectives such as "intuitive" and "easy" to characterize using the mouse to control these functions. The locomotion task used these functions most intensely, and control schemes that used the mouse had the highest scores. The preference for mouse input perhaps not surprising, given that prior research that also found mouse input to be faster and more accurate than HMD input [26, 31, 40, 59, 64, 87].

However, the preference for mouse input was not universal, and again there was a minority who enjoyed the interaction of aiming with head movement. In general, there were some who enjoyed using the HMD to target or to control camera view because they found it more immersive and "VR-like".

Dead zone. Whether the control scheme had a dead zone, and the dead zone's attributes such as size or shape, was another factor that affected the player experience. While the presence of a dead zone did not affect player preferences, 
player feedback uncovered some usability issues. Many participants found that the dead zone control schemes made the controls less responsive for the locomotion task. For instance, they would move the mouse left or right to turn, but the mouse would not affect steering inside the dead zone. In contrast, the dead zone did not seem to negatively affect usability scores for the targeting task, perhaps because the mouse as opposed to the HMD was used to aim, the former which was the more important factor influencing usability for targeting.

The summary above highlights that the type of control scheme had an impact on outcomes. Overall, usability, immersion, and control schemes preferences were in most cases uniformly related. The one exception is that control scheme did not have a significant effect on the time efficiency measure. Participants maintained a consistent speed regardless of the control scheme, the impact of which was that they would incur either more or fewer errors. This finding suggests that players do not choose slow down and be more cautious in response to control schemes that offer less precise control. 


\section{Chapter 4: User Study 2}

In this chapter we describe user study 2 that evaluated the usability of virtual reality control schemes for FPS controller-based locomotion games using the Oculus Touch motion controls. While the input devices used in user study 1 (mouse and keyboard) are still in use, there are more modern alternatives. In 2016, Oculus released the Oculus Touch [61] motion controllers. We conducted another study to measure their impact on control scheme usability since it has not been addressed by other researchers to date.

\subsection{Participants}

Twenty-eight participants were recruited through SONA, posters, social media, and word-of-mouth. Two participants chose not to finish the study because of cybersickness ( 2 females). Thus, the analysis is based on data from 26 participants (19 male). The mean age of the participants was 20; the range was 18 to 30 .

The participants were English-speaking and over 18 years old. As for study 1, we recruited experienced $3 \mathrm{D}$ gamers, as participants need to have a basic level of comfort with gaming in 3D space. We used the Game Experiences Questionnaire as a 
screening tool in which prospective participants were asked to rate their skill in playing $3 \mathrm{D}$ games on a scale of 1 to 5 (with 5 being very skilled) in answer to the question: "What is your skill level in playing games set in a $3 \mathrm{D}$ world from a first person viewpoint.” The mean skill level for the sample was 3.3.

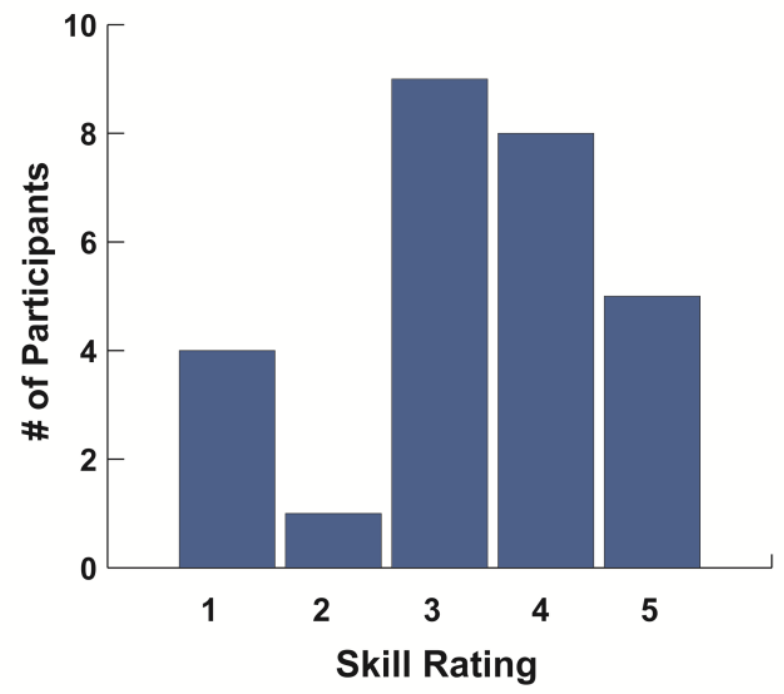

Illustration 17. Distribution of player skill ratings, study 2.

We also collected information about the platforms and input devices that participants typically used to play games. Of the 28 participants, 15 had used motion controllers for PC or console games, 19 had used gamepad, 19 had used mouse and keyboard. Only 1 participant hadn't used any of the aforementioned methods and was an exclusively mobile gamer. Only 1 participant was a frequent VR gamer. The remainder of the sample had either never used VR or only tried it once or twice. 


\subsection{Apparatus}

\subsubsection{VR Game Hardware}

For the present study, we used a PC gaming setup that included a desktop PC with Intel i7-7700, 4.20GHz CPU with $32 \mathrm{MB}$ RAM and two Nvidia GeForce 1080 video cards in a dual-card SLI setup.

To this standard gaming system we added an Oculus Rift CV1 and the Oculus Touch Motion Controllers. The Oculus Touch's left thumbstick translates the player avatar in all directions (forward, back, left and right); the right thumbstick is mapped to rotating the player avatar and in the coupled control scheme it also rotates the camera view, as seen in Illustration 18 . Players aim at targets in the game by pointing at them with the motion controller, similar to aiming using an arcade light gun.
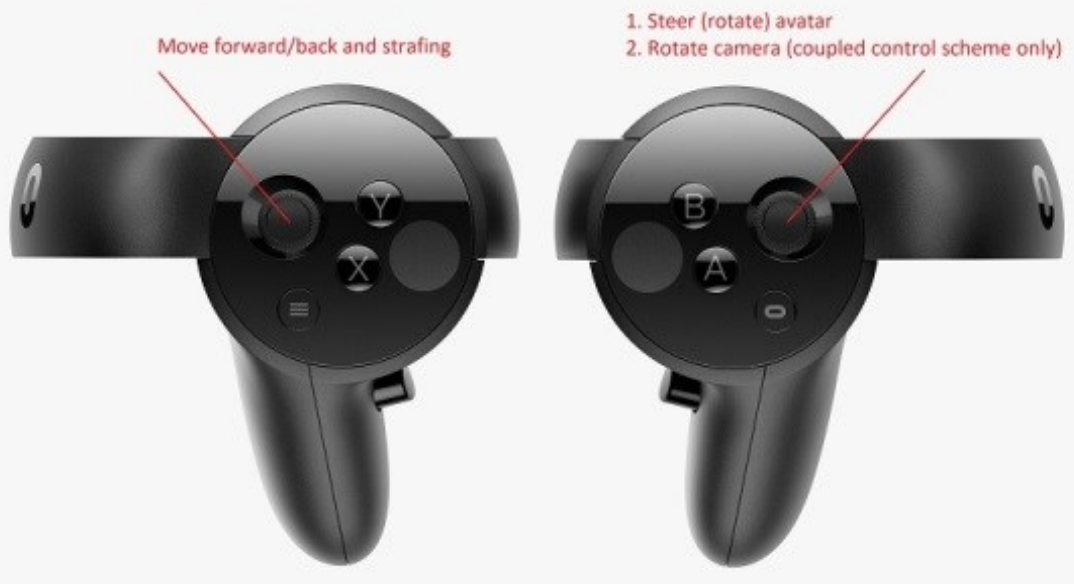

Illustration 18. Oculus Touch control mappings. 


\subsubsection{VR Game Software}

User study 2 used a virtual-reality enabled videogame called Serious Sam: The First Encounter (SS:TFE) [21]. This is a game in the first-person shooter genre, released by Croteam in 2009. We chose this game for several reasons: (1) it comes with the Serious Sam Fusion Editor 2017, a level editing tool by Croteam [22] that we used to create custom test environments, and (2) $S S$ : TFE has two control schemes (a coupled and a decoupled scheme) defined for use in virtual reality mode.

\subsubsection{Control Schemes}

Serious Sam: The First Encounter (SS:TFE) comes with pre-defined control schemes. In the options menu, the player can choose either a "head-oriented" or "hand-oriented" control scheme. Hand-oriented is equivalent to a decoupled control scheme where the player can look around freely without affecting movement direction. Head-oriented is equivalent to a coupled control scheme where the player moves in the direction of the camera.

In this section, we will explain how the each control scheme functions. We have also provided tables that show the control-display mappings for each input axis, showing the relationship between each control and display degree-of-freedom, for each component of the locomotion interaction technique.

\subsubsection{Coupled Control Scheme}

The coupled control scheme in SS:TFE is called the "head-oriented" control in the options menu. With the coupled control scheme, HMD input is coupled to the right joystick so that they control the same functions. Consequently, the player can choose to use either the right joystick or the HMD (or both as they see fit). Coupled control 
schemes are similar to the standard "mouselook" control scheme used in present-day games. However, with the addition of motion controllers, targeting is decoupled from the camera view, as the position of the motion controllers are tracked and targeting is achieved by ray-casting forward from the motion controller position and orientation. The player essentially "points" the motion controller toward the desired target. See Table 10.

\begin{tabular}{|c|c|c|c|c|c|c|c|c|c|c|c|}
\hline \multicolumn{4}{|c|}{ Viewpoint Control } & \multicolumn{4}{|c|}{ Movement Direction } & \multicolumn{4}{|c|}{ Targeting } \\
\hline \multirow{2}{*}{$\begin{array}{l}\text { Input } \\
\text { Device }\end{array}$} & \multicolumn{3}{|c|}{ Control-Display Mappings } & \multirow{2}{*}{$\begin{array}{l}\text { Input } \\
\text { Device }\end{array}$} & \multicolumn{3}{|c|}{ Control-Display Mappings } & \multirow{2}{*}{$\begin{array}{l}\text { Input } \\
\text { Device }\end{array}$} & \multicolumn{3}{|c|}{ Control-Display Mappings } \\
\hline & Axis & Control & Display & & Axis & Control & Display & & Axis & Control & Display \\
\hline \multirow{6}{*}{$\begin{array}{l}\text { Right } \\
\text { joystick }\end{array}$} & $x$ & & & \multirow{6}{*}{$\begin{array}{l}\text { Right } \\
\text { joystick }\end{array}$} & $x$ & & & \multirow{6}{*}{$\begin{array}{l}\text { Motion } \\
\text { Controll } \\
\text { er }\end{array}$} & $x$ & $\bullet$ & $\longrightarrow$ \\
\hline & $y$ & & & & $y$ & & & & $y$ & $\bullet$ & $\longrightarrow$ \\
\hline & $z$ & & & & $z$ & & & & $z$ & $\bullet$ & $\longrightarrow$ \\
\hline & $\theta_{x}$ & & $\bullet$ & & $\theta_{x}$ & & $\bullet$ & & $\theta_{x}$ & $\bullet$ & $\longrightarrow$ \\
\hline & $\theta_{y}$ & & b & & $\theta_{y}$ & & ל & & $\theta_{y}$ & $\bullet$ & $\longrightarrow$ \\
\hline & $\theta_{z}$ & & & & $\theta_{z}$ & & & & $\theta_{z}$ & $\bullet$ & $\longrightarrow$ \\
\hline \multirow[t]{6}{*}{ HMD } & $x$ & & $\longrightarrow$ & \multirow[t]{6}{*}{ HMD } & $x$ & & $\longrightarrow$ & & $x$ & & \\
\hline & $y$ & $\bullet$ & $\longrightarrow$ & & $y$ & $\bullet$ & $\longrightarrow$ & & $y$ & & \\
\hline & $z$ & & $\longrightarrow$ & & $z$ & & $\longrightarrow$ & & $z$ & & \\
\hline & $\theta_{x}$ & & $\longrightarrow$ & & $\theta_{x}$ & & $\longrightarrow$ & & $\theta_{x}$ & & \\
\hline & $\theta_{y}$ & $\bullet$ & $\longrightarrow$ & & $\theta_{y}$ & $\bullet$ & $\longrightarrow$ & & $\theta_{y}$ & & \\
\hline & $\theta_{z}$ & $\bullet$ & $\longrightarrow$ & & $\theta_{z}$ & $\bullet$ & $\longrightarrow$ & & $\theta_{z}$ & & \\
\hline
\end{tabular}

Table 10. Control-display mappings for the coupled control scheme, study 2.

\subsubsection{Decoupled Control Scheme}

The decoupled control scheme in SS:TFE is called the "hand-oriented" control in the options menu. Unlike coupled controls, decoupled schemes assign different functions to the right joystick and the HMD. In this scheme, the right joystick controls steering, but the HMD controls the camera view. This scheme allows the player to look around the environment independent from the avatar's movement direction. Targeting is decoupled from camera view and avatar movement direction, as described above for the coupled control scheme. See Table 11. 


\begin{tabular}{|c|c|c|c|c|c|c|c|c|c|c|c|}
\hline \multicolumn{4}{|c|}{ Viewpoint Control } & \multicolumn{4}{|c|}{ Movement Direction } & \multicolumn{4}{|c|}{ Targeting } \\
\hline \multirow{2}{*}{$\begin{array}{l}\text { Input } \\
\text { Device }\end{array}$} & \multicolumn{3}{|c|}{ Control-Display Mappings } & \multirow{2}{*}{$\begin{array}{l}\text { Input } \\
\text { Device }\end{array}$} & \multicolumn{3}{|c|}{ Control-Display Mappings } & \multirow{2}{*}{$\begin{array}{l}\text { Input } \\
\text { Device }\end{array}$} & \multicolumn{3}{|c|}{ Control-Display Mappings } \\
\hline & Axis & Control & Display & & Axis & Control & Display & & Axis & Control & Display \\
\hline \multirow[t]{6}{*}{ HMD } & $x$ & $\bullet$ & $\longrightarrow$ & \multirow{6}{*}{$\begin{array}{l}\text { Right } \\
\text { joystick }\end{array}$} & $x$ & & & \multirow{6}{*}{$\begin{array}{l}\text { Motion } \\
\text { Controll } \\
\text { er }\end{array}$} & $x$ & $\bullet$ & $\longrightarrow$ \\
\hline & $y$ & $\bullet$ & $\longrightarrow$ & & $y$ & & & & $y$ & $\bullet$ & $\longrightarrow$ \\
\hline & $z$ & $\bullet$ & $\longrightarrow$ & & $z$ & & & & $z$ & $\bullet$ & $\longrightarrow$ \\
\hline & $\theta_{x}$ & $\bullet$ & $\longrightarrow$ & & $\theta_{x}$ & & & & $\theta_{x}$ & $\bullet$ & $\longrightarrow$ \\
\hline & $\theta_{y}$ & $\bullet$ & $\longrightarrow$ & & $\theta_{y}$ & & $\checkmark$ & & $\theta_{y}$ & $\bullet$ & $\longrightarrow$ \\
\hline & $\theta_{z}$ & $\bullet$ & $\longrightarrow$ & & $\theta_{z}$ & & & & $\theta_{z}$ & $\bullet$ & $\longrightarrow$ \\
\hline
\end{tabular}

Table 11. Control-display mappings for the decoupled control scheme, study 2.

\subsubsection{Task-based Game Maps}

The research team made two custom game maps using the Serious Sam Fusion Editor 2017, a tool by Croteam [22]. These custom maps test specific user tasks, given that task influences a control scheme's impact on variables of interest [12, 56]. We henceforth refer to these maps as the "locomotion" map and the "gaming context" map.

The locomotion map tests how agile the player is when using a given control scheme. The player must move his or her character from the start point to end point while avoiding collision with maze walls. The maze was scripted to deduct 1 point from the player's health, for each second that the player touched the wall (Health was initially set to 100 at the start of the game). Each hit point (HP) lost is counted as an "error". 


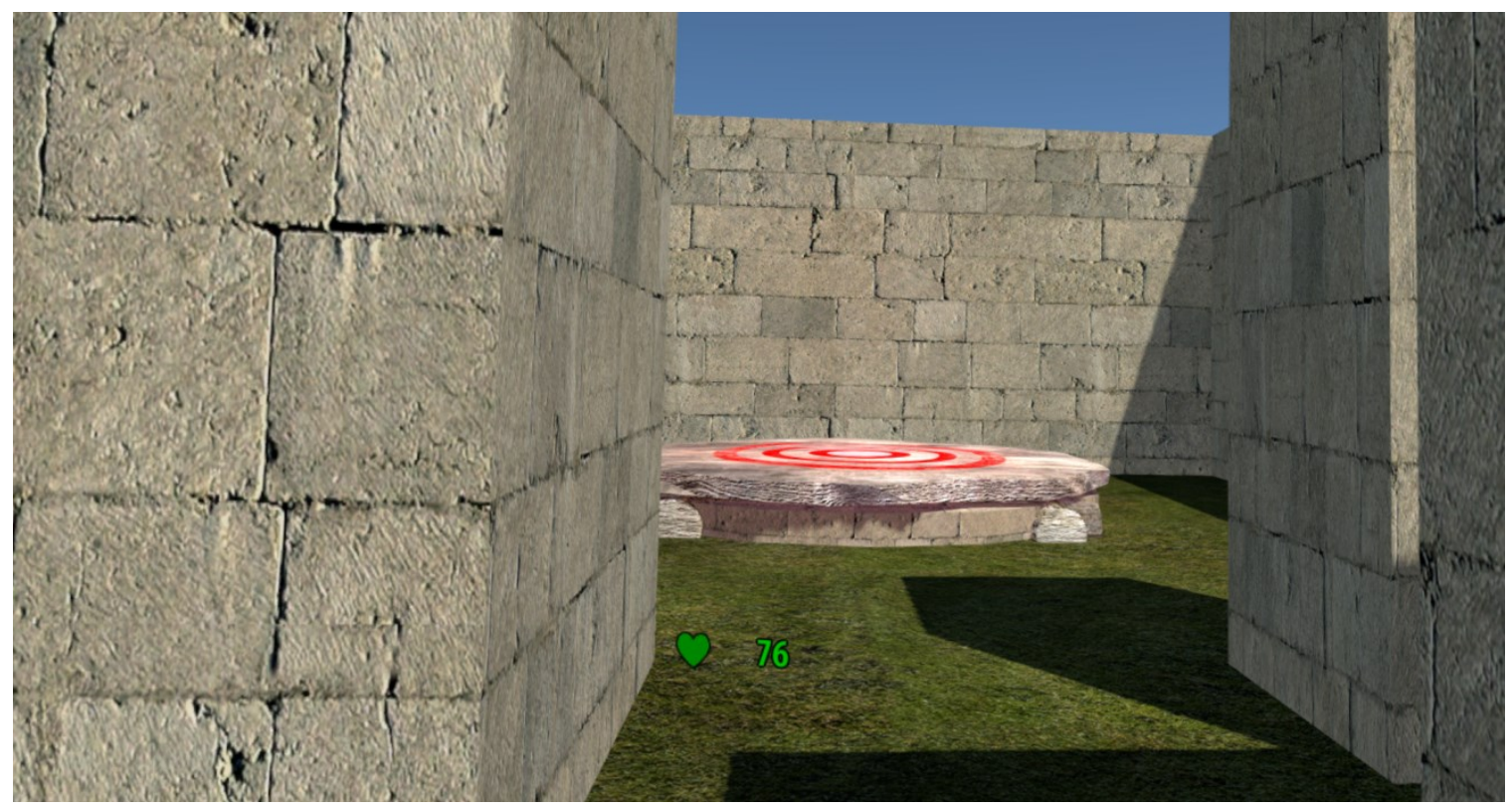

Illustration 19. First-person view of the locomotion map, study 2.

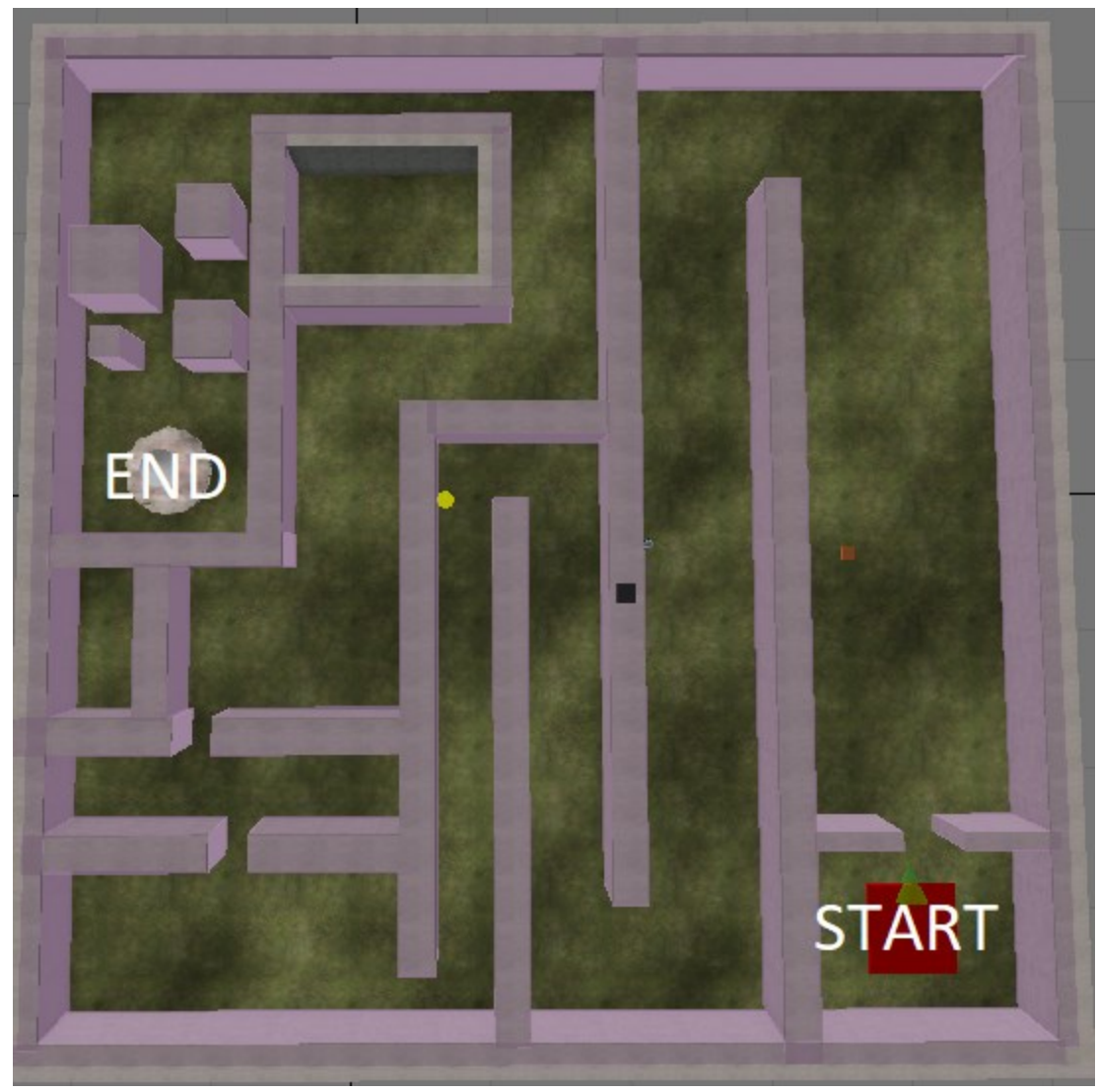

Illustration 20. Top-down view of the locomotion map from the Serious Editor. 
The gaming context map provides an ecologically valid test of the control schemes, by measuring participants' performance as they played a real game level. We chose the Bends on Sand level from SS:TFE in survival mode (see Illustration 21). In this level, the player shoots as many enemies as he or she can while avoiding the attacks of the AI-controlled characters. The number of kills and survival time were logged.

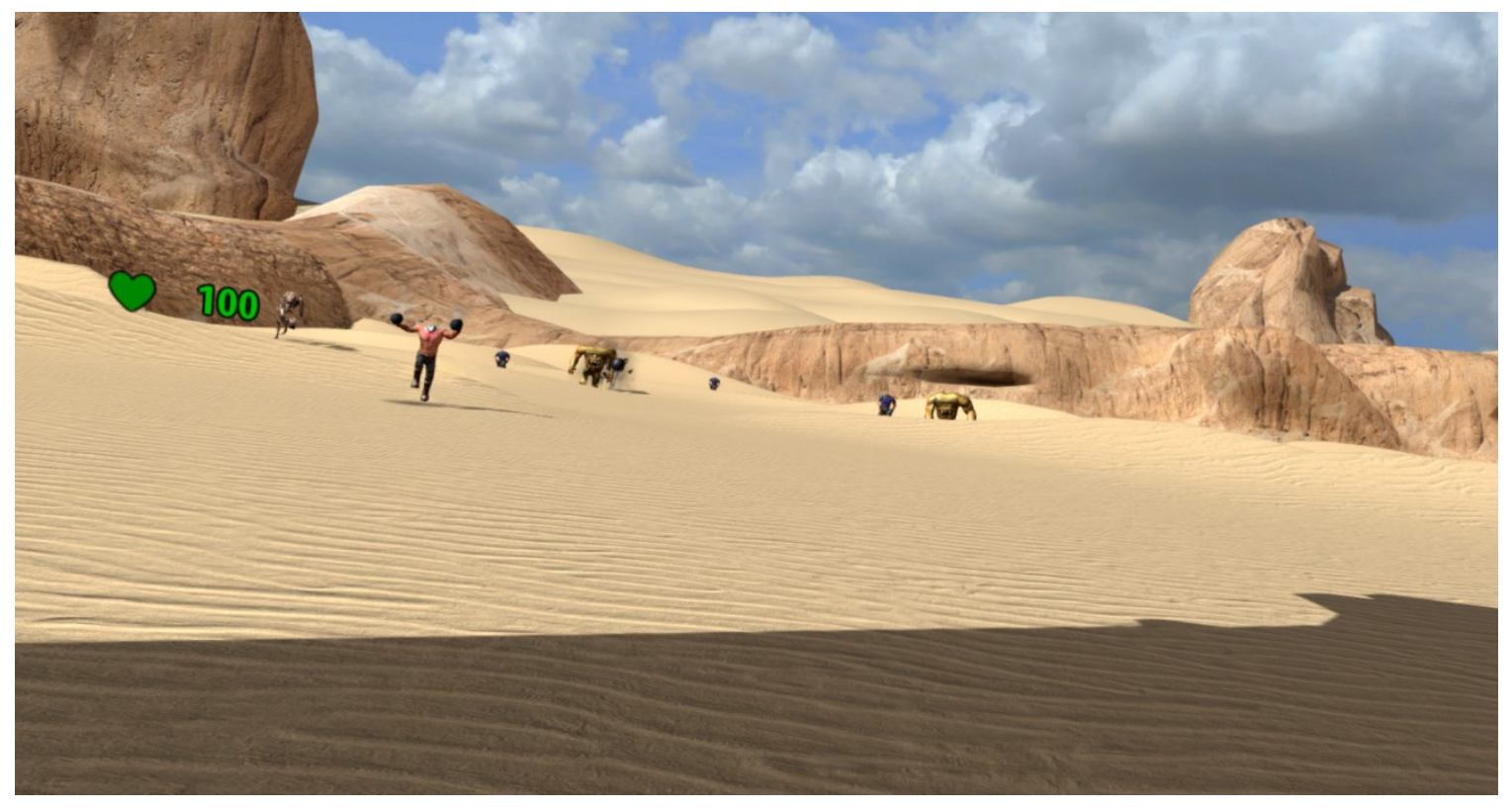

Illustration 21. In-game view of the Bends on Sand level.

\subsection{Design}

In order to measure the effect of control scheme, we conducted an experiment with a $2 \times 2$ within-subjects design. The two independent variables (IV) were: (1) control scheme, with two levels: decoupled and coupled; and (2) task, with two levels: locomotion and gaming context. There was an additional IV for the gaming context task only: trial, which tracked usability metrics across four trials. In summary, there were two VR control schemes and two tasks, all participants experienced all four 
conditions, and the gaming context task repeatedly measured each control scheme four times.

To counterbalance possible order effects, we alternated the order of each control scheme for each participant (to reduce the number of possible orders, we did not alternate the two tasks). Table 12 shows the two possible orderings for the control schemes.

\begin{tabular}{|c|c|c|c|}
\hline Order set & Task & \multicolumn{2}{|c|}{ Control scheme } \\
\hline \multirow{2}{*}{ Order 1} & Gaming context & Control 1 & Control 2 \\
\hline & Locomotion & Control 1 & Control 2 \\
\hline \multirow{2}{*}{ Order 2} & Gaming context & Control 2 & Control 1 \\
\hline & Locomotion & Control 2 & Control 1 \\
\hline
\end{tabular}

Table 12. Counterbalancing schedule, study 2.

\subsubsection{Data Analysis}

For data collection and analysis, we relied on the same mixed-methods approach that we used for study 1 (shown again in this chapter in Illustration 22). We gathered quantitative data on usability, immersion, and player preference for each control scheme. To provide insight into the quantitative results, we also obtained qualitative feedback from the participants on each control scheme. For a full explanation of our process for combining qualitative and quantitative insights in this study, see Chapter 3 , as we used a similar methodology and analysis for both studies. 


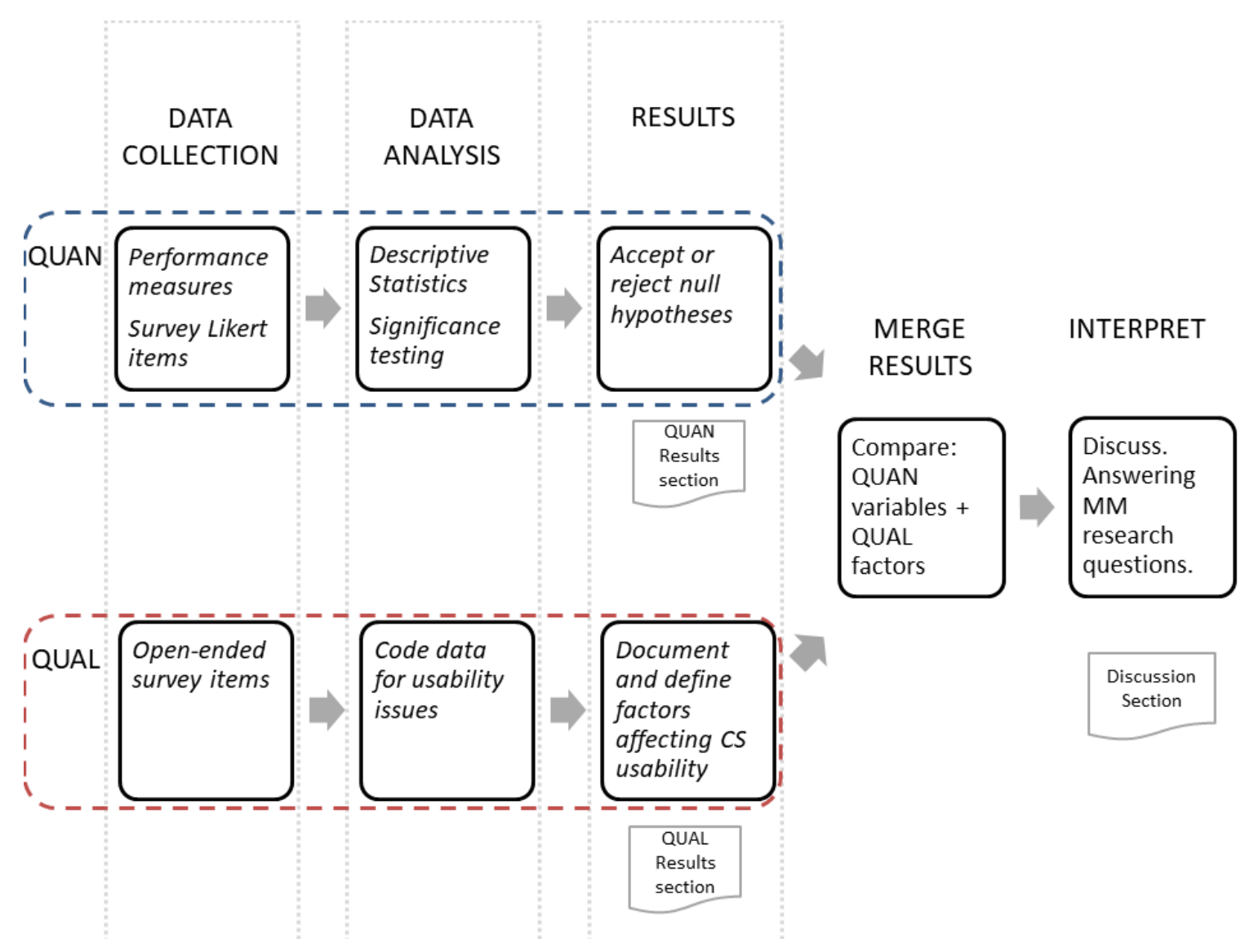

Illustration 22. Process flow for convergent parallel mixed-methods

\subsection{Measures}

To accurately assess how each control scheme affected participants, researchers collected quantitative and qualitative data measuring usability and immersion constructs.

\subsubsection{Usability Measures}

We tracked participants' performance on effectiveness and efficiency measures (described in more detail in Chapter 3) by tracking error rates and time for the locomotion task and kills and survival time in the gaming context task. Satisfaction 
with each control scheme was measured through self-report questionnaire using both quantitative and qualitative methods.

We added a new usability metric in study 2: learnability. Learnability is a dimension of usability proposed by Nielson [33, 78], which is defined as "How easy is it for users to accomplish basic tasks the first time they encounter the design." We measured learnability by measuring four trials of the gaming context task and compared usability scores over time. Usability measures are summarized in Table 13.

\begin{tabular}{|c|c|c|c|c|}
\hline Data Type & Variable & Measures Used & Collection Method & Data Analysis \\
\hline \multirow{4}{*}{ QUAN } & Effectiveness & Performance scores & $\begin{array}{l}\text { Locomotion Task: Error rate } \\
\text { (Derived from number of } \\
\text { collisions with walls) } \\
\text { Gaming Task: Enemies killed } \\
\text { ("kills") and survival time. }\end{array}$ & Paired t-test \\
\hline & Efficiency & Performance scores & $\begin{array}{l}\text { Locomotion Task: Time to } \\
\text { complete maze }\end{array}$ & Paired t-test \\
\hline & Learnability & Performance scores & $\begin{array}{l}\text { Gaming Task: Track } \\
\text { effectiveness and efficiency } \\
\text { scores over } 4 \text { trials. }\end{array}$ & $\begin{array}{l}\text { 2-way ANOVA with } \\
\text { control scheme and } \\
\text { trial as IV }\end{array}$ \\
\hline & Satisfaction & $\begin{array}{l}\text { Task-Specific Control } \\
\text { Scheme } \\
\text { Questionnaire, } \\
\text { and; } \\
\text { Control Scheme } \\
\text { Preference } \\
\text { Questionnaire }\end{array}$ & $\begin{array}{l}\text { Preference score: Players were } \\
\text { asked to rank the control } \\
\text { schemes in order of } \\
\text { preference. } \\
\text { "Fun" score: rated on a scale of } \\
1-10 \\
\text { "Naturalness" score: rated on a } \\
\text { scale of } 1-10\end{array}$ & $\begin{array}{l}\text { Preference score: } \\
\text { Wilcoxon rank. } \\
\text { Fun and Naturalness } \\
\text { scores: paired t-tests }\end{array}$ \\
\hline QUAL & Satisfaction & $\begin{array}{l}\text { Task-Specific Control } \\
\text { Scheme } \\
\text { Questionnaire, } \\
\text { and; } \\
\text { Control Scheme } \\
\text { Preference } \\
\text { Questionnaire }\end{array}$ & $\begin{array}{l}\text { Both questionnaires included } \\
\text { open-ended questions that } \\
\text { asked players to give feedback } \\
\text { about each control scheme and } \\
\text { explain the reasons for their } \\
\text { control scheme preference } \\
\text { rankings. }\end{array}$ & $\begin{array}{l}\text { Qualitative analysis: } \\
\text { Identify player } \\
\text { feedback relating to } \\
\text { satisfaction. }\end{array}$ \\
\hline
\end{tabular}

Table 13. Usability measures, collection method and data analysis, study 2. 


\subsubsection{Task-specific Control Scheme Questionnaire}

We developed a new questionnaire for study 2, which replaced the semi-structured interviews that we conducted in study 1 to gathered feedback on each control scheme. The 4-item Task-Specific Control Scheme Questionnaire gathers qualitative and quantitative data on participant's control scheme experiences in that task context (Appendix D). The questionnaire contained three open-ended questions gathered positive and negative feedback about each control scheme in the context of each task, which we added to our qualitative data corpus. As well as two Likert-type questions asking participants to rate "fun" and "naturalness" of each scheme on a scale of 1 to 5 ;

\subsubsection{Control Scheme Preferences Questionnaire}

The 4-item Control Scheme Preferences Questionnaire was also used in study 1, and was designed to gather participants' impressions of the control schemes overall, not in the context of a specific task (Appendix C). This self-report questionnaire is administered once, at the end of the testing session, after participants have played $S S: T F E$ with all control schemes.

\subsubsection{Immersion}

As in study 1, we will use Jennet et al.'s Immersive Experiences Questionnaire [15] (Appendix D), to measure immersion while using each control schemes during the gaming context task as well as identifying player feedback about immersion in qualitative data. See Table 14 for a summary of immersion measures. 


\begin{tabular}{|c|l|l|l|l|}
\hline Data Type & Variable & \multicolumn{1}{|c|}{ Measures Used } & Collection Method & Analysis \\
\hline QUAN & Immersion & $\begin{array}{l}\text { Immersive } \\
\text { Experiences } \\
\text { Questionnaire }\end{array}$ & Likert rating scale & Paired t-test \\
\hline QUAL & Immersion & $\begin{array}{l}\text { Task-Specific Control } \\
\text { Scheme } \\
\text { Questionnaire } \\
\text { Control Scheme } \\
\text { Preferences } \\
\text { Questionnaire }\end{array}$ & $\begin{array}{l}\text { Players were asked to give } \\
\text { feedback about each control } \\
\text { scheme and explain the } \\
\text { reasons for their control } \\
\text { scheme preferences. }\end{array}$ & $\begin{array}{l}\text { Qualitative analysis: } \\
\text { Identify player } \\
\text { feedback relating to } \\
\text { immersion. }\end{array}$ \\
\hline
\end{tabular}

Table 14. Immersion measures, collection method and analysis, study 2.

\subsection{Procedure}

Sessions were conducted individually in a computer research lab that was equipped with hardware needed for experiment as outlined in Apparatus section. Participants were asked to sign a consent form after arrival, then given a quick verbal introduction to the study procedures and aims. We also shared information about cybersickness and instructed participants to inform researchers if they were uncomfortable at any point. Next, participants filled out the Game Experience Questionnaire.

The first phase of the experiment allowed participants to acclimatise to VR. To begin, participants took a pre-test Cybersickness Rating Scale. Then they were fitted with the Oculus Rift CV1 headset and played the Karnak Temple level (See Illustration 23) using the control scheme planned for the first trial (we used complete counterbalancing so the orders in which the control schemes were presented for each participant was pre-set). They could use the control scheme for as long as it took to feel comfortable (this took approximately 2 to 3 minutes). After acclimatisation, we applied the Cybersickness Rating Scale once more to screen for cybersickness, after 
which we asked players verbally about cybersickness symptoms. At this point, two participants chose to drop out of the study.

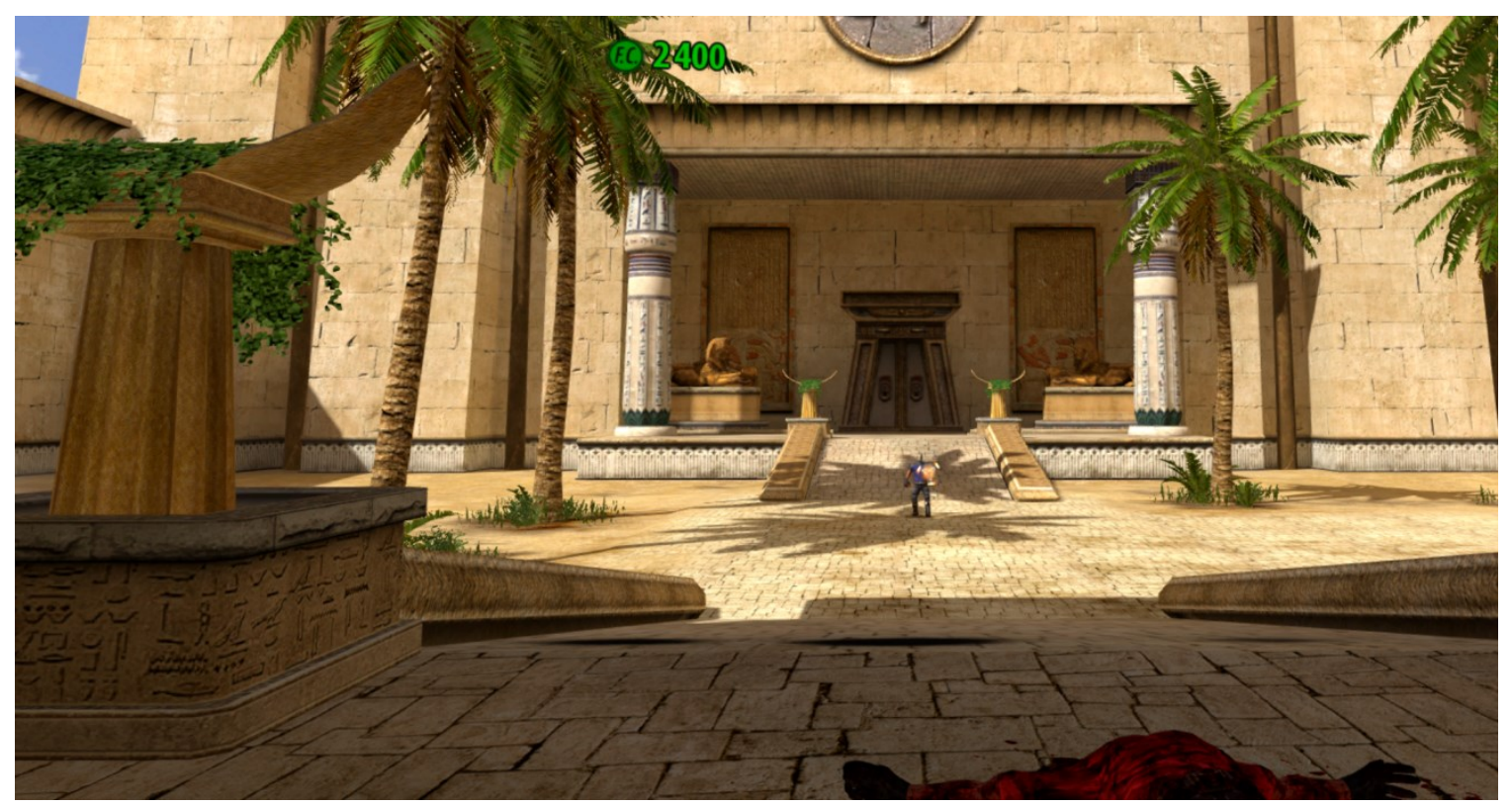

Illustration 23. The Karnak Temple level, which participants played to acclimatize to VR.

In the next phase of the experiment, participants complete four trials of the game context map task using the Bend on Sand level, for each of the two control schemes. The order in which we present the control schemes and tasks is explained in the Counterbalancing section below. After the game context task, participants completed the Immersive Experiences Questionnaire for each control scheme. (i.e., there were two applications of the IEQ per test session).

Next, participants completed one trial of the locomotion map-one trial per control scheme. We recorded each participant's usability scores (time, number of errors) for each condition. Participants completed the Task-Specific Control Scheme Questionnaire (TCSQ) after using each scheme. Therefore, there were two applications of the TCSQ per experimental session. 
At the end of the test session, participants filled out the Control Scheme Preference Questionnaire. The study session took approximately 60 minutes to complete. Participants were given course credit or $\$ 10$ as compensation for their time.

\subsection{Results}

We begin with the quantitative results related to usability, immersion, and control scheme preference. Recall that our primary independent variable was impact of control scheme; where appropriate, some analysis also included a second variable, namely the effect of time. After the quantitative results, we present the qualitative analysis of the survey data.

\subsubsection{Usability Results}

We had two tasks, locomotion and gaming context. Since these tasks were not comparable, as for study 1 , we ran separate analyses for each task and dependent variables related to that task. Specifically, for the locomotion task, to determine the effect of control scheme (coupled or decoupled) on the effectiveness and efficiency usability metrics, we ran separate paired sample t-tests for each dependent variable (time and errors). For the gaming context task, to determine the effect of control scheme on the effectiveness and learnability usability metrics, we ran separate twoway within-subjects ANOVA for each dependent variable (survival time and kills) with control scheme and trial as the independent variable. The trial IV assessed learnability over a series of 4 measurements of the DV per participant. For this latter analysis, when Mauchley's test of sphericity was significant, we followed the standard convention of reporting test statistics using the Greenhouse-Geisser adjustment. 

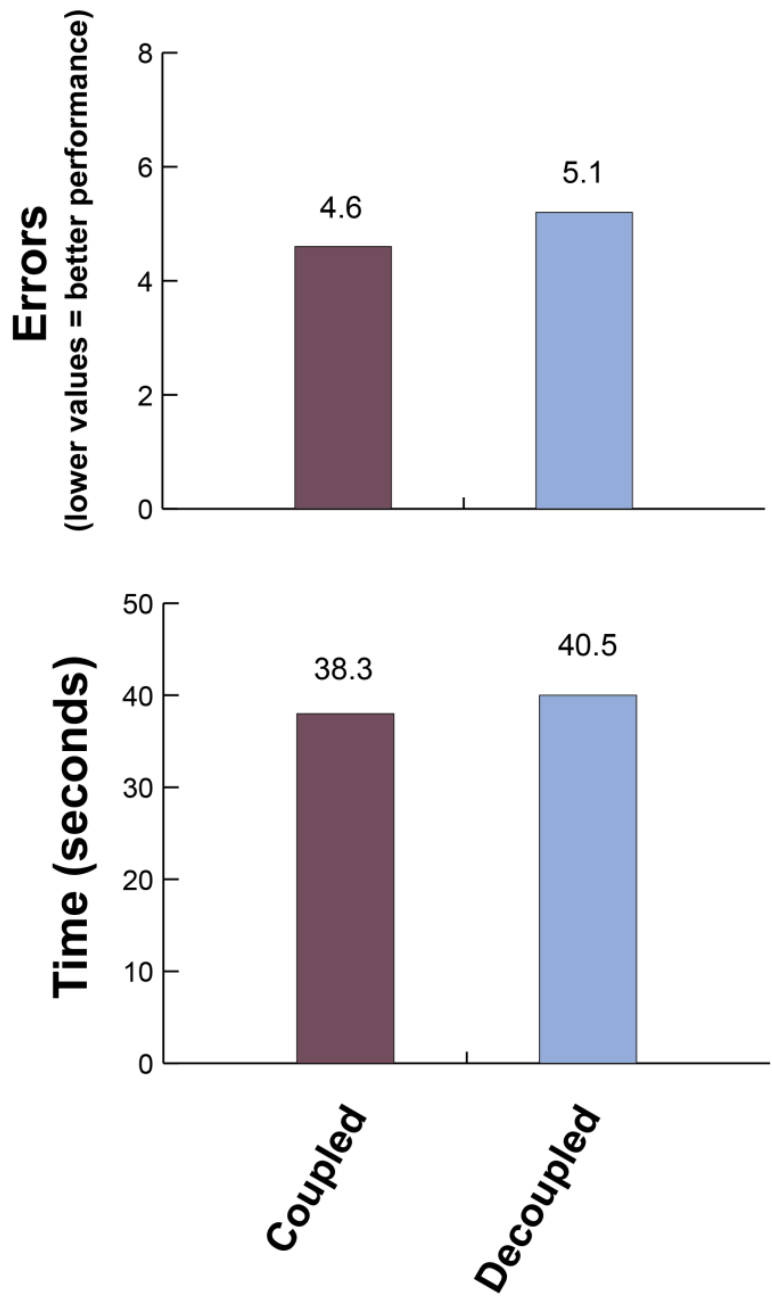

Illustration 24. Locomotion task mean time and errors by control scheme, study 2

\subsubsection{Locomotion task: Errors}

We begin with the descriptive statistics for our efficiency measure, total errors. Illustration 24 shows that while on average participants made more errors with the decoupled control scheme $(M=$ $5.15, S D=5.66)$ than with the coupled scheme $(M=4.62, S D=$ 4.32), a paired samples t-test did not find a significant difference, $t(25)=-.82, p=.418$, and represented a small effect size, $d=$ .11.

\subsubsection{Locomotion Task: Time}

Illustration 24 shows that, on average, time spent performing the locomotion task with the decoupled scheme $(M=$ 40.58, $S D=17.74)$ was higher than when using the coupled scheme $(M=38.31, S D=$ 14.41). However, a paired samples t-test did not find a significant difference, $t(25)=-$ $1.145, p=.263$, and represented a small effect size, $d=.14$. 


\subsubsection{Gaming Task - Kills}

Illustration 25 shows the descriptive stats for the two control schemes in the gaming context task, measured by mean kills, over 4 trials. A two-way within-subjects ANOVA confirmed that there was no significant main effect of control scheme on the number of kills averaged over 4 trials, $F(1,25)=.158, p=.694, \boldsymbol{\eta}_{\mathrm{p}}{ }^{2}=.006$. There was also no significant main effect of trial on kills per control scheme, $F(2.22,55.61)=$ $.625, p=.555, \boldsymbol{\eta}_{p}{ }^{2}=.024$. This analysis tested learnability and so the results show that participants' performance did not vary significantly over time.

\subsubsection{Gaming Task - Survival Time}

Illustration 25 shows the descriptive stats for the two control schemes in the gaming context task, measured by mean survival time, over 4 trials. A two-way withinsubjects ANOVA confirmed that there was no significant main effect of control scheme on mean survival time averaged over the four trials, $F(1,25)=.024, p=.879$, $\boldsymbol{\eta}_{\mathrm{p}}{ }^{2}=$.001. There was also no significant main effect of trial on kills per control scheme, $F(2.76,69.09)=2.02, p=.118, \eta_{p^{2}}=.075$, which was our learnability metric, indicating that there was no learning effect.
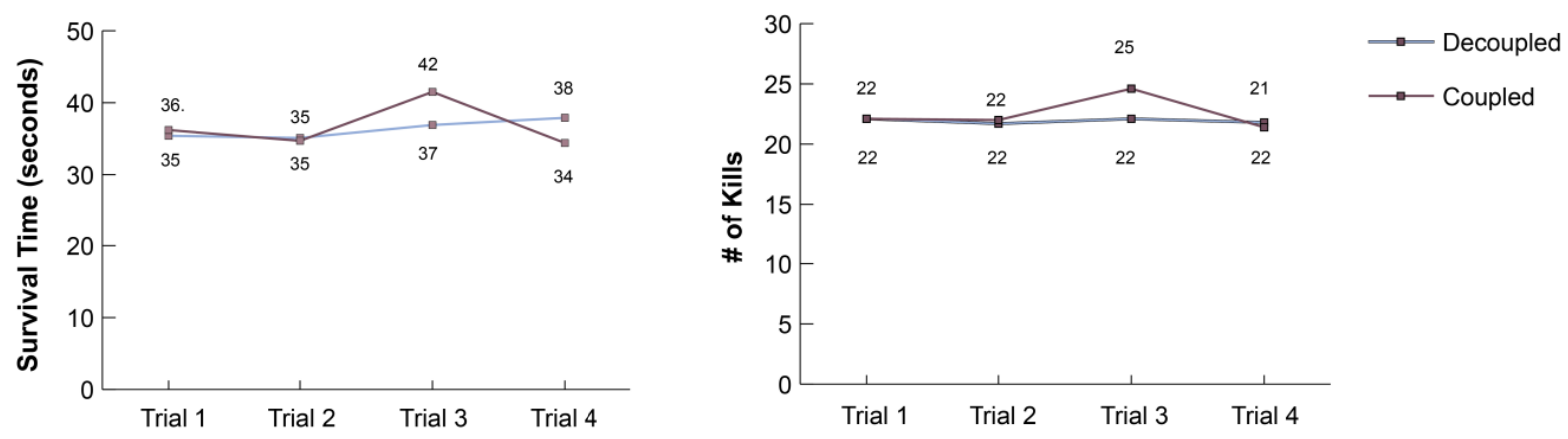

Illustration 25. Gaming task mean survival time and kills by control scheme, study 2. 


\subsubsection{Immersion Results}

Participants filled out the Immersive Experiences Questionnaire (IEQ) after playing the gaming context task for each control scheme. The questionnaire produced two immersion scores per participant: (1) an immersion score, which was obtained by summing the ratings for the 31 questions in the IEQ, and (2) the "single question measure of immersion", in which the participant rated how immersed they felt on a
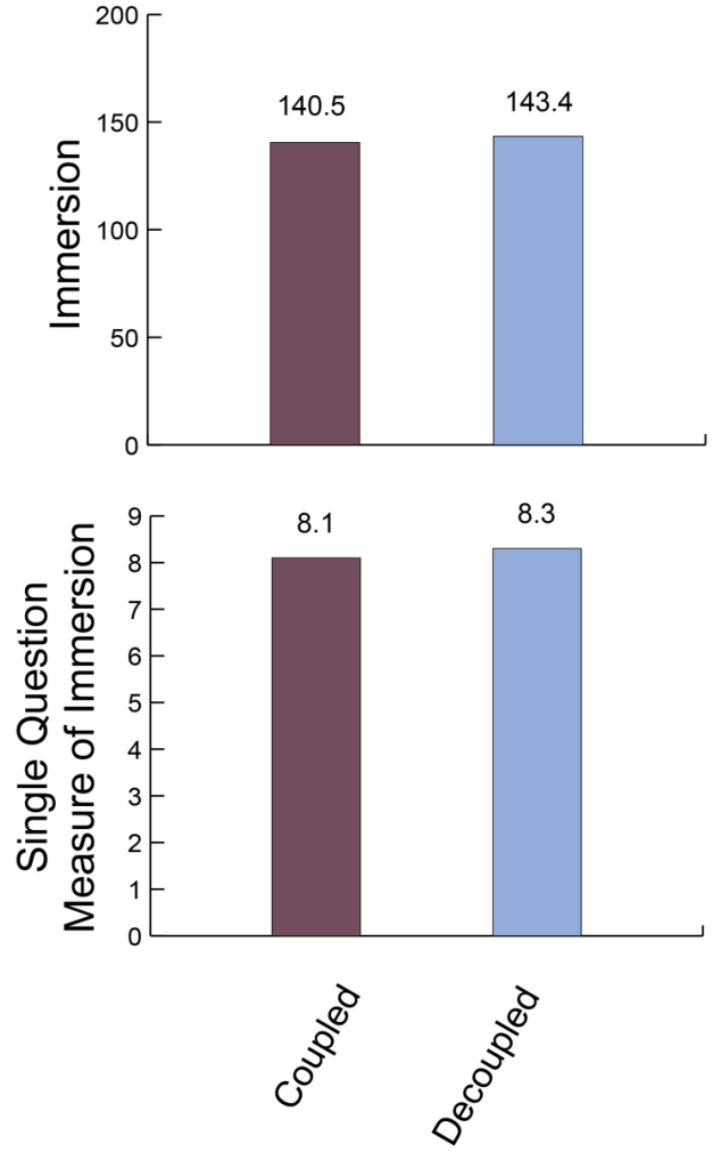

Illustration 26. Mean immersion (top) and single question measure of immersion scores (bottom), by control scheme type, study 2 scale of 1 to 10.

As the descriptive data Illustration 26 shows, participants rated the decoupled control scheme $(M=143.42, S D=11.91)$ as slightly more immersive than the coupled scheme $(M=140.46, S D=$ 11.43). However, a paired samples t-test did not find a significant difference, $t(25)=-1.19, p=.247$, and represented a small effect size, $d=.25$.

The above result for the immersion scores was mirrored in our confirmatory analysis of the single question measure of immersion (i.e., "How immersed did you feel?”). A paired samples t-test confirmed that there was no significant difference in mean single question of 
immersion ratings for the coupled $(M=140.46, S D=11.43)$ and decoupled $(M=$ 143.42, $S D=11.91)$ control schemes, $t(25)=-1.04, p=.247, d=.08$.

\subsubsection{Preference Results}

To determine which control scheme players preferred, we analyzed the control scheme rankings from the Control Scheme Preference Questionnaire to get a preference score for each control scheme type. As shown in Table 15, 14 participants preferred the coupled scheme and 12 participants preferred the decoupled scheme. Rankings were compared using a Wilcoxon ranked-sign nonparametric test, which was not significant, $z=-.392, p=.841$.

\begin{tabular}{|c|c|c|}
\hline Scheme & Ranked 1 $^{\text {st }}$ & Ranked 2 $^{\text {nd }}$ \\
\hline Coupled & $14(53.8 \%)$ & $12(46.1 \%)$ \\
\hline Decoupled & $12(46.1 \%)$ & $14(53.8 \%)$ \\
\hline
\end{tabular}

Table 15. Frequency table for control scheme rankings, study 2.

We also asked participants to rate the naturalness and fun of each control scheme. The naturalness ratings for the coupled scheme $(M=6.88, S D=2.40)$ was higher than for the decoupled scheme $(M=6.85, S D=2.88)$. A paired samples t-test did not find a significant difference in mean naturalness ratings, $t(25)=-.104, p=.918$, and represented a small effect size, $d=.01$. While the fun ratings for the decoupled scheme $(M=8.08, S D=2.02)$ was higher than for the coupled scheme $(M=8.04$, $S D=1.78)$ a paired samples t-test did not find a significant difference, $t(25)=.081, p$ $=.936$, and represented a small effect size, $d=.02$. 


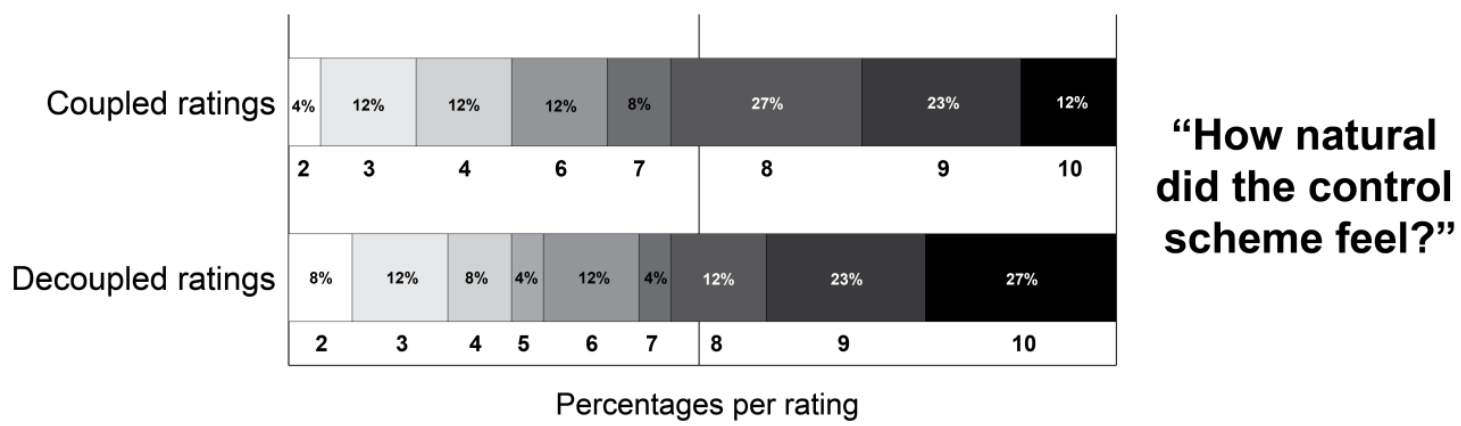

Illustration 27. Distribution of "naturalness" ratings, by control scheme.

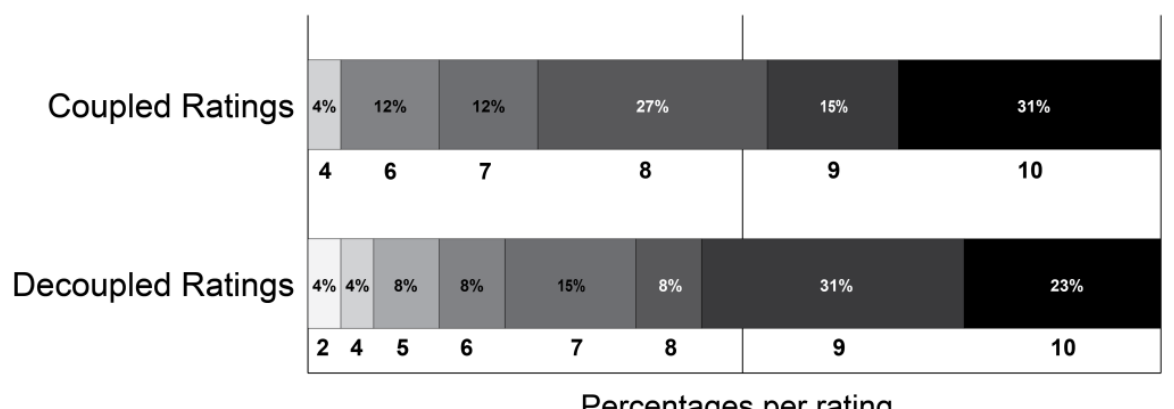

"How fun

was using the control scheme?"

Illustration 28. Distribution of "fun" ratings, by control scheme.

\subsection{Qualitative Analysis}

To provide deeper insight into the quantitative results, we also obtained qualitative feedback from the participants on each control scheme. As was the case for study 1 , we thought it was important to include qualitative data in the study to better understand the reasons behind the quantitative results and to identify what players value in a VR control scheme. 


\subsubsection{Coupled Controls Scheme Impressions}

The feedback about the coupled control scheme was mostly favorable. Recall that in coupled control schemes, both the HMD and right joystick are mapped to controlling the camera and steering the avatar. The player can choose to use either input device.

The most common sentiments were that the coupled scheme was easy to use, natural, immersive, and accurate. Seven participants found the control scheme was easy to use ( $\left.\mathrm{P}_{3}, \mathrm{P}_{4}, \mathrm{P}_{7}, \mathrm{P} 10, \mathrm{P}_{11}, \mathrm{P}_{14}, \mathrm{P}_{15}\right)$, which was the most common feedback for the coupled scheme. For most of these participants, the coupled controls allowed them to easily determine what direction they will move in (P3, P4, P10, P11, P14). P11 said: "I felt more in control and I felt like I could react faster because moving my head controlled my direction”. Participants generally viewed the coupling of head and joystick control for steering and camera positively (P9, P17, P18, P20, P24), several of which liked the novelty of the interaction: "you have 2 different ways of controlling the way you move and it was cool having 2 ways." (p9). Participants also found steering their avatar with their head movements a practical choice, calling this method of interaction more accurate than using joystick controls ( $\left.\mathrm{P}_{5}, \mathrm{P}_{12}, \mathrm{P} 20\right)$, because it "allowed for small adjustments when moving my head" (P12); more immersive (P12, P25), because of the body based "natural" movements, "I could use my actual face to move around the imaginary world which added to the feeling that I was part of it.” (P25). In fact, participants (P1, P9, P11, P22) found the coupled controls incorporated more natural movements into the control scheme, in ways that affected the control of their character: "moving my head controlled my direction and I feel like that is a natural reflection.” (P11). 
Of the negative feedback received about the coupled controls, 4 participants felt the control scheme was hard to control, finding the coupling of head and joystick control for steering and camera less usable because of confusion between head and hand input (P16, P20, P23, P25), as participant 20 states: "I wasn't always moving my head but when I was, I felt it was a little frustrating that I would always move in the direction that I'm looking. If checking a corner I would have to look, then turn my head back in order to move forward." (P23).

\subsubsection{Decoupled Control Scheme Impressions}

The feedback for the decoupled control scheme was not as uniformly positive as for the coupled scheme: while many participants found it easy to use, citing the scheme's familiarity, almost as many found it hard to use. Recall that in decoupled control schemes, the user could look around freely while steering their avatar with the right joystick.

The most common sentiment for the decoupled control scheme was that it was easy to use, with 9 participants $\left(\mathrm{P}_{7}, \mathrm{P} 9, \mathrm{P} 11, \mathrm{P} 12, \mathrm{P} 14, \mathrm{P} 16, \mathrm{P}_{17}, \mathrm{P} 22, \mathrm{P} 23\right)$ feeling this way. Many participants liked how the steering and camera controls were controlled by different input devices: with the head controlling the camera and the right joystick

controlling steering (P14, P16, P17, P23), finding it "filters out the confusion of having directional movement with both looking and joystick." (P14). Many participants found using the right joystick for steering without interference from head movements (as in the coupled scheme) was a familiar experience for them ( $\mathrm{P} 2$, P3, P6, P14, P17, P23, P25). Participant 17 said, "I felt that it was a little simpler and straight forward. Perhaps that is because I grew up relying on controllers for in-game 
movement, rather than swiveling my head." (P23). The fact that head movement did not control steering was also cited as an advantage for detecting threats in the game world (P4, P23). Participant 23 said, "I rely on the controllers to move around while having the freedom to use my head, to scan the field of view." (P23).

On the other hand, an almost equal number of participants found the decoupled control scheme hard to control (P2, P6, P7, P11, P15, P16, P17, P24, P25). The most common culprit was participant's awareness of their avatar's facing direction would get out of sync with their head direction, causing them to have to recalibrate ( $\mathrm{P} 2$, P10, P11, P16, P24). Participant 2 said, "If there was a way to see where my body was facing (like seeing my legs for example) I think I would have preferred this control scheme. The main drawback was forgetting where the front of my body was facing if I turned my head around.” (p24). There were also some easily-corrected usability issues with this scheme. Participants found the right joystick's sensitivity was too high (P1, P2, P8, P17, P22), which may have affected participant's judgements (although the sensitivity can be modified in the options.) This control scheme also constrained the right joystick movement rotation only, which felt restrictive and is not the normal behaviour; however, only a couple of participants mentioned this (p6, P25). As participant 25 explained, "My biggest problem with this scheme was that I couldn't look up and down using my controller (like I would normally be able to do using console controllers); it felt like I was confound to some kind of horizontal movement path where the only way to look up was using my actual face. Changing that would give the player MUCH more control over his character." If our maps required shooting enemies high or low, it is possible that constraining joystick control to only rotation would affect player's performance. 


\subsection{Discussion}

In user study 2 we compared coupled and a decoupled control schemes in a free locomotion VR game setting using motion controllers as input device. We hypothesized that the coupled control scheme would be more usable, based on the results of study 1 , which showed that the coupled scheme was more usable and more preferred by our participants. However, the quantitative results from study 2 showed no significant effect of control scheme on our usability metrics: efficiency, effectiveness and satisfaction. Nevertheless, the qualitative results revealed a more complex picture. While the coupled scheme had overwhelmingly positive feedback, the participants reported some usability issues with the decoupled control scheme, the most common issue being participants finding the separation of the avatars movement direction and camera view confusing and inefficient. The usability issues inherent in the decoupled control scheme did not prevent approximately half (12) of the participants from preferring that scheme (whereas just over half, i.e., 14, participants preferred the coupled scheme overall). Our hypothesis that the decoupled scheme would be more immersive because the camera view is only controlled by the head mounted display input was not confirmed in either the quantitative immersion scores (which did not show a significant effect of control scheme on immersion) or qualitative feedback from participants.

Study 2 tested the control schemes in two contexts, as prior work has shown that control schemes can perform differently for different VR tasks. We chose a locomotion task, where the player's agility using the control schemes was the focus, and an ecologically valid gaming context task where the player had to fight and defend against enemies. We hypothesized that the coupled control scheme would 
have significantly better usability scores for both tasks, but the results were nonsignificant for the quantitative usability metrics of both tasks.

As a mixed-methods study, our goal was not only to measure usability, but to generate some best-practice recommendations for control scheme design. Based on qualitative analysis of the feedback across the two control schemes, we identified two control scheme factors that players liked and disliked about control schemes for VR controller-based locomotion games. These usability factors can be of use to game designers when creating novel control schemes or choosing a control scheme. The usability factors affecting the usability of the control and their implications are as follows:

1. Whether steering and camera control were coupled or decoupled.

2. Whether the input device DOF was constrained.

We discuss each of these factors below, integrating our qualitative and quantitative results where appropriate.

Coupled vs. Decoupled controls. Our analysis suggests that overall, the most important factor affecting player sentiment was whether the control scheme was coupled or decoupled. Although the usability, preference and immersion metrics were close between the two schemes, the qualitative data revealed usability issues with the decoupled scheme. The feedback for the coupled scheme was almost wholly positive, while the feedback for the decoupled scheme was mixed, with most of the negative feedback centering on how the decoupled nature of the controls. Recall that in the decoupled scheme, the camera was controlled by the HMD, while steering the avatar was controlled by the right joystick (whereas the coupled scheme camera and 
steering were coupled and controlled by both the joystick and HMD devices). When using the decoupled scheme participants simply had difficulty remembering which direction their avatar was facing (controlled by the right joystick) in relation to the direction their head (and the camera) was facing. When their awareness of their avatar's facing direction got out of sync with their head direction, players had to stop and think for a moment to reorient themselves, which they found inefficient. However, several participants liked how the steering and camera controls were controlled by different input devices finding that while using the coupled scheme head movement could unintentionally interfere with steering their avatar with the right joystick. Overall, the coupled scheme had consistently positive feedback and is the recommended control scheme for action-oriented controller-based locomotion VR games.

Input device degrees of freedom. Another important factor was whether the control scheme constrained the degrees of freedom on an input device. Participants felt constrained and confused by not being able to move the camera up or down with the right joystick, forcing them to use their heads to move the camera up or down when using the decoupled control scheme. This could have affected usability even more if our game maps placed enemies above or below the player's eyeline as they would be forced to target using head movement to target, which prior research has found is less accurate than mouse or joystick input [43-46].

The summary above shows that while there was no significant main effect of control scheme for our usability and immersion metrics, the qualitative results tell a slightly different story. While the decoupled scheme was preferred by 12 participants, the inherent decoupled-ness of the scheme caused usability issues for some, as it was 
difficult for some participants to maintain awareness of their avatar's facing direction. The coupled scheme was the most usable as judged by consistently positive feedback and the absence of major usability issues reported for this scheme. The importance of gathering user data in addition to usability metrics is therefore highlighted. 


\section{Chapter 5: Conclusions and Limitations}

In this thesis, we conducted two user studies investigating the usability of coupled and decoupled control schemes for controller-based locomotion in FPS VR games. In user study 1, described in Chapter 3, we evaluated three control schemes types: coupled, decoupled and dead zone, using keyboard and mouse as input devices. We measured the effect of control scheme type on usability and immersion. We found that familiar, "coupled" control schemes that: (1) coordinated control of the camera view and steering the avatar, (2) had no dead zone (i.e., "keyhole" or area of the screen in which input is not recognized), and (3) allowed players to use the mouse to control all in-game functions were preferred and resulted in the best usability and immersion scores. In user study 2, discussed in Chapter 4, we evaluated a coupled and a decoupled control scheme using the Oculus Touch motion controller as the input device, and with a more complex gaming context task. The quantitative results of study 2 found no significant difference between the coupled and decoupled control schemes on our usability or immersion metrics. In contrast to these results, however, (and mirroring the results of study 1) the qualitative analysis uncovered usability issues associated with the decoupled control scheme. Many participants found the decoupled scheme difficult to use, whereas the coupled scheme had more 
consistently positive feedback. Thus, the qualitative data made it possible to uncover the usability issues inherent in the decoupling of camera and steering control. To summarize, across both studies, the coupled controls were the most usable when taking all of the data into account.

As mentioned above, the quantitative results showed a significant effect of control scheme for study 1, but not study 2. For the "kills" measures, this could be due to using different input devices for targeting in the studies. In study 1, the coupled control scheme mapped the more precise mouse input $[27,59,87]$ to targeting, whereas the decoupled scheme mapped the HMD to targeting, which may account for the significant difference between the control schemes for this measure. In contrast, in study 2, the non-significant effect of control scheme on the kills measure may have been due to targeting sharing the same input device, the Oculus Touch motion controllers, which were furthermore decoupled from camera view.

Another possible explanation for why study 1 and study 2 had different quantitative results lies in player behaviour. Recall that in the coupled scheme, players have the option of choosing either the HMD or the mouse (or the right joystick for study 2) to steer their avatar. One limitation of our study is that we did not track the behaviour of participants to ascertain which input device they used. Player behaviour may have affected our results in various ways. One possibility is that participants who used head steering for the coupled scheme in study 2 may have increased error rates during the locomotion task-whereas if they used the joystick instead we might expect results more similar to study 1 . This fact highlights the importance of tracking behaviour when more than one input method can be used. 
Early versions of the Oculus Rift HMD, such as the DK2 we used in study 1, were not capable of room-scale VR. Therefore, another difference is that participants were seated in study 1, whereas study 2 used an updated version of the Oculus Rift (the CV1) where users could `stand and move around the tracked environment using a room-scale VR system. This may have allowed users to make adjustments to their position and rotation using body pose. This could conceivably make the decoupled control scheme more usable, as some prior research has shown that real walking results in fewer errors $[17,79,85]$, although results have been insignificant when compared to controller-based travel in other studies [58].

A further limitation of this work is that the results may not be generalizable to games that use other input devices. Studies have found that input device has an effect on the player experience $[1,55]$. When comparing the results of study 1 and study 2 , it is obvious that the control schemes for study 2, which used motion controllers, received more positive feedback from participants than study 1 , which used the mouse and keyboard. The devices used may explain the difference in acceptance. As we saw in study 1 , the input device was one of the significant factors we found to affect usability and controls that used the mouse for steering or targeting had higher performance and preference scores. However, a single study that evaluates control schemes using different input devices and with targeted questionnaires could tease out the different control scheme preferences for each device, an approach that Bowman [8] took in the test bed they created for testing various control schemes and device types for locomotion and targeting tasks in virtual environments, albeit not in a gaming context. 
As mentioned above, the control schemes we selected were defined by the developers of Half Life 2: Deathmatch and Serious Sam: the First Encounter VR. Although two categories of schemes (the coupled and decoupled schemes) are among the most commonly found in present day VR games, other control schemes may replace them as VR games mature. Future directions could then include testing a more diverse set of VR control schemes.

Another limitation is the lack of control we had in specifying the values for the control scheme's control-display relationships. As we did not create the control schemes, it is possible that the control schemes have other differences that we could not detect. Programmers at Valve and Croteam may have used different values for transfer functions. where "the amount of cursor motion depends on the velocity of mouse motion or controller motion." [49], or implemented dead zones differently These factors are difficult or impossible to ascertain from the information given to the public about the controls by Croteam and Valve programmers, but may have differences that affect user's performance [20]. In study 2, for example, participants found the joystick sensitivity for the coupled scheme too high, which may have affected usability. A true comparison between schemes is therefore difficult to make, although we attempt to make general conclusions in this thesis.

While our study is a first step towards designing control schemes for FPS VR games, more research is needed into how game genre affects a control scheme's impact. As far as game genre, due to the need to choose a set of tasks to determine cause and effect relationships between control schemes and outcomes of interest, our environment included standard game elements like locomotion and targeting. While in study 2, we were able to include an ecologically valid FPS gaming context task, our 
study focused on FPS gameplay in particular. Thus, future work should investigate the impact of control schemes in other types of games that require different types of tasks. Another interesting extension includes exploring the impact of control schemes on the targeting task per se, using more standardized aiming task such as the Fitt's Law reciprocal selection task widely used in evaluating computer pointing devices $[71,76]$.

Finally, this research does not differentiate between novice and experienced gamers. Experienced gamers are accustomed to using the mouselook control scheme and the results of the studies show that they are biased in favor of similar control schemes. While gamers are the target market for VR games at this time, the results cannot be generalized to inexperienced or first-time gamers. It would therefore be interesting to discover which controls are most usable for populations who do not have preexisting biases. 


\section{Appendices}

\section{Appendix A : User Study 1 Results}

This appendix presents the complete results for all 6 control schemes evaluated in user study 1 without collapsing them into control scheme types.

\begin{tabular}{|c|c|c|c|c|}
\hline DV & $\begin{array}{c}\text { Control } \\
\text { Scheme } \\
\text { Type }\end{array}$ & $\begin{array}{l}\text { Control } \\
\text { Scheme }\end{array}$ & Mean & Effect of Control Scheme \\
\hline \multirow{6}{*}{$\begin{array}{l}\text { Locomotion } \\
\text { - Errors }\end{array}$} & \multirow{2}{*}{ Coupled } & Control 0 & 1.69 & \multirow[t]{6}{*}{$\begin{array}{l}\text { 1-way ANOVA: F }(2.621,91.751)=7.061, \mathrm{p}<.001, \boldsymbol{\eta}_{\mathrm{p}} 2= \\
.168\end{array}$} \\
\hline & & Control 6 & 1.61 & \\
\hline & Decoupled & Control 1 & 3.89 & \\
\hline & \multirow{3}{*}{$\begin{array}{l}\text { Dead zone } \\
\quad(\mathrm{DZ})\end{array}$} & Control 2 & 2.61 & \\
\hline & & Control 3 & 2.17 & \\
\hline & & Control 4 & 2.11 & \\
\hline \multirow{6}{*}{$\begin{array}{l}\text { Locomotion } \\
\text { - Time }\end{array}$} & \multirow{2}{*}{ Coupled } & Control 0 & 46.34 & \multirow{6}{*}{$\begin{array}{l}\text { 1-way ANOVA: F }(3.574,125.09)=.243, \mathrm{p}<.896, \boldsymbol{\eta}_{\mathrm{p}} 2= \\
.007)\end{array}$} \\
\hline & & Control 6 & 45.84 & \\
\hline & Decoupled & Control 1 & 44.76 & \\
\hline & \multirow{3}{*}{$\begin{array}{l}\text { Dead zone } \\
\quad \text { (DZ) }\end{array}$} & Control 2 & 44.58 & \\
\hline & & Control 3 & 47.36 & \\
\hline & & Control 4 & 45.84 & \\
\hline \multirow{2}{*}{$\begin{array}{l}\text { Targeting } \\
\text { Kills }\end{array}$} & \multirow{2}{*}{ Coupled } & Control 0 & 24.39 & \multirow{2}{*}{$\begin{array}{l}\text { 1-way ANOVA: }\left(\mathrm{F}(3.541,123.92)=10.240, \mathrm{p}<.001, \boldsymbol{\eta}_{\mathrm{p}} 2\right. \\
=.226)\end{array}$} \\
\hline & & Control 6 & 27.06 & \\
\hline
\end{tabular}




\begin{tabular}{|c|c|c|c|c|}
\hline & Decoupled & Control 1 & 23.11 & \\
\hline & \multirow{3}{*}{$\begin{array}{l}\text { Dead zone } \\
\text { (DZ) }\end{array}$} & Control 2 & 27.47 & \\
\hline & & Control 3 & 27.94 & \\
\hline & & Control 4 & 27.36 & \\
\hline \multirow{6}{*}{$\begin{array}{l}\text { Immersion } \\
\text { score }\end{array}$} & \multirow{2}{*}{ Coupled } & Control 0 & 152.42 & \multirow{6}{*}{$\begin{array}{l}\text { 1-way ANOVA: } \mathrm{F}(3.023,105.8)=4.411, \mathrm{p}<.006, \boldsymbol{\eta}_{\mathrm{p}} 2= \\
.112\end{array}$} \\
\hline & & Control 6 & 155.50 & \\
\hline & Decoupled & Control 1 & 142.50 & \\
\hline & & Control 2 & 150.39 & \\
\hline & $\begin{array}{l}\text { Dead zone } \\
\text { (DZ) }\end{array}$ & Control 3 & 152.56 & \\
\hline & & Control 4 & 153.42 & \\
\hline & & Control 0 & 3.5 & Friedman's test: $\chi 2(5)=12.136, p=0.033$ \\
\hline & & Control 6 & 3.72 & \\
\hline Control & Decoupled & Control 1 & 2.83 & \\
\hline & & Control 2 & 3.42 & \\
\hline & $\begin{array}{l}\text { Dead zone } \\
\text { (DZ) }\end{array}$ & Control 3 & 3.67 & \\
\hline & & Control 4 & 3.64 & \\
\hline
\end{tabular}

Table 16. Complete usability results for all 6 control schemes, study 1. 


\section{Appendix B Game Experience Questionnaire}

This questionnaire was administered at the beginning of the test session. It verified that the participant is an experienced gamer and gathers basic demographic data.

1. Age:

2. Gender: (male, female, prefer to self-describe)

3. What is your skill level in playing games set in a $3 \mathrm{D}$ world from a first person viewpoint? (Not at all skilled 12345 Very skilled)

4. Have you ever played a virtual reality game or used a head-mounted display such as the Oculus Rift? (Never, Only a few times, Seldom, Frequently)

5. What platform(s) do you play games on? Check all that apply: (Console, PC, Tablet or mobile, Other)

6. What game controllers do you typically use? Choose all that apply: (mouse and keyboard, Gamepad, Motion controllers, Touchscreen, Other) 


\section{Appendix C Control Scheme Preferences Questionnaire}

This questionnaire will be administered once at the end of the test session to gather the participant's general impressions of the VR control schemes.

1. Please rank the control schemes from the most preferred to least preferred. (i.e., your favorite control scheme would be first, and rank the rest in descending order)

2. Why do you prefer the control scheme you ranked first more than the other?

3. Why do you like the control scheme you ranked last the least?

4. If you have any other comments about any of the control schemes used in this study, please write them below. 


\section{Appendix D Immersive Experiences Questionnaire}

This questionnaire was created by Jennet et al. (2008) [37] and is designed to measure player immersion and gather subjective impressions on the control scheme. We will administer this questionnaire after each play session. There are three play sessions in the study; in each play session, the player experiences one of three control schemes.

1. To what extent did the game hold your attention? (Not at all $1234567 \mathrm{~A}$ lot)

2. To what extent did you feel you were focused on the game? (Not at all 12345 67 A lot)

3. How much effort did you put into playing the game? (Not at all $1234567 \mathrm{~A}$ lot)

4. Did you feel that you were trying your best? (Not at all 1234567 Very much so)

5. To what extent did you lose track of time. E.g., did the game absorb your attention so that you were not bored? (Not at all 1234567 A lot)

6. To what extent did you feel consciously aware of being in the real world while playing? (Not at all 1234567 Very much so)

7. To what extent did you forget about your everyday concerns? (Not at all 123 4567 A lot)

8. To what extent were you aware of yourself in your surroundings? (Not at all 1 234567 Very aware)

9. To what extent did you notice events taking place around you? (Not at all 123 4567 A lot) 
10. Did you feel the urge at any point to stop playing and see what was happening around you? (Not at all 1234567 Very much so)

11. To what extent did you feel that you were interacting with the game environment? (Not at all 1234567 Very much so)

12. To what extent did you feel that you were separated from your real-world environment? (Not at all 1234567 Very much so)

13. To what extent did you feel that the game was something fun you were experiencing, rather than a task you were just doing? (Not at all 1234567 Very much so)

14. To what extent did your sense of being in the game environment stronger than your sense of being in the real world? (Not at all 1234567 Very much so)

15. To what extend did you feel so involved that you were unaware that you were even using any controls? E.g., it was effortless. (Not at all 1234567 Very much so)

16. To what extent did you feel that you were moving through the game according to your own will? (Not at all 1234567 Very much so)

17. To what extent did you find the game challenging? (Not at all 1234567 Very difficult)

18. Were there any times during the game in which you just wanted to give up? (Not at all $1234567 \mathrm{Alot}$ )

19. To what extent did you feel motivated while playing? (Not at all $1234567 \mathrm{~A}$ lot)

20. To what extent did you find the game easy? (Not at all 1234567 Very much so) 
21. To what extent did you feel like you were making progress towards the end of the map? (Not at all $1234567 \mathrm{Alot}$ )

22. How well do you think you performed in the game? (Very poorly 1234567 Very well)

23. To what extent did you feel emotionally attached to the game? (Not at all 123 4567 Very much so)

24. To what extent were you interested in seeing how the game's events would progress? (Not at all $1234567 \mathrm{Alot})$

25. How much did you want to "win" the game? (Not at all 1234567 Very much so)

26. Were you in suspense about whether or not you would do well in the game? (Not at all 1234567 Very much so)

27. At any point did you feel yourself become so involved that you wanted to speak to the game directly? (Not at all 1234567 Very much so)

28. To what extent did you enjoy the graphics and the imagery? (Not at all 1234 567 A lot)

29. How much would you say you enjoyed playing the game? (Not at all 123456 7 A lot)

30. When it ended, were you disappointed that the game was over? (Not at all 12 34567 Very much so)

31. Would you like to play the game again? (Definitely no 1234567 Definitely yes)

32. How immersed did you feel? (Not at all immersed 12345678910 Very immersed) 


\section{Appendix E Task-Specific Control Scheme Questionnaire}

This questionnaire will be administered 2 times. Its purpose is to gather impressions of how the control schemes function in the locomotion task.

1. How natural did it feel to use the coupled/decoupled control scheme? (Not natural at all 12345678910 Very natural)

2. How fun was it to use the coupled/decoupled control scheme? (Not at all fun 1 2345678910 Very fun)

3. What did you like about using the coupled/decoupled control scheme for the maze map?

4. What did you dislike about using the coupled/decoupled control scheme for the maze map?

5. If you have any other thoughts about the coupled/decoupled control scheme, please write them below 


\section{References}

[1] Abeele, V. Vanden et al. 2013. More Naturalness, Less Control : The Effect of Natural Mapping on the Co-located Player Experience. Foundations of Digital Games (2013), 1-9.

[2] Anthes, C. et al. 2004. Navigation models for a flexible, multi-mode VR navigation framework. Virtual Reality Continuum and its Applications (New York, New York, USA, 2004), 476.

[3] Bakker, N.H. et al. 2003. Effects of head-slaved navigation and the use of teleports on spatial orientation in virtual environments. Human Factors. 45, 1 (2003), 160-169. DOI:https://doi.org/10.1518/hfes.45.1.160.27234.

[4] Boletsis, C. 2017. The New Era of Virtual Reality Locomotion: A Systematic Literature Review of Techniques and a Proposed Typology. Multimodal Technologies and Interaction. 1, 4 (2017), 24.

DOI:https://doi.org/10.339o/mti1040024.

[5] Boletsis, C. and Cedergren, J.E. 2019. VR Locomotion in the New Era of Virtual Reality: An Empirical Comparison of Prevalent Techniques. Advances in Human-Computer Interaction. 2019, (Apr. 2019), 1-15. DOI:https://doi.org/10.1155/2019/7420781. 
[6] Boletsis, C. and Cedergren, J.E. 2019. VR Locomotion in the New Era of Virtual Reality: An Empirical Comparison of Prevalent Techniques. Advances in Human-Computer Interaction. April (2019). DOI:https://doi.org/10.1155/2019/7420781.

[7] Bolte, B. et al. 2011. The Jumper Metaphor: An Effective Navigation Technique for Immersive Display Setups. VRIC (2011), 6-8.

[8] Bowman, D.A. et al. 1998. A methodology for the evaluation of travel techniques for immersive virtual environments. Virtual Reality. 3, 2 (Jun. 1998), 120-131.

[9] Bowman, D.A. 1999. Interaction Techniques for Common Tasks in Immersive Virtual Environments.

[10] Bowman, D.A. et al. 1999. Maintaining spatial orientation during travel in an immersive virtual environment. Presence: Teleoperators and Virtual Environments. 8, 6 (Dec. 1999), 618-631.

[11] Bowman, D.A. 2002. Principles for the Design of Performance-oriented Interaction Techniques. Handbook of Virtual Environments. 277-300.

[12] Bowman, D.A. et al. 2001. Testbed evaluation of virtual environment interaction techniques. Presence: Teleoperators and Virtual Environments. 10, 1 (2001), 75-95.

[13] Bowman, D.A. et al. 1997. Travel in immersive virtual environments: an evaluation of viewpoint motion control techniques. Virtual Reality (1997), $45^{-52 .}$

[14] Bowman, D.A. and Hodges, L.F. 1999. Formalizing the design, evaluation, and application of interaction techniques for immersive virtual 
environments. Visual Languages \& Computing. 10, 1 (1999), 37-53.

[15] Bozgeyikli, E. et al. 2016. Point \& Teleport locomotion technique for virtual reality. CHI PLAY (2016), 205-216.

[16] Brown, E. and Cairns, P. 2004. A grounded investigation of game immersion. $C H I$ (2004), 1-8.

[17] Bruder, G. and Steinicke, F. 2014. Threefolded motion perception during immersive walkthroughs. VRST. (2014), 177-185.

DOI:https://doi.org/10.1145/2671015.2671026.

[18] Bungie Software 1994. Marathon.

[19] Cairns, P. et al. 2014. The influence of controllers on immersion in mobile games. Proceedings of the 32nd annual ACM conference on Human factors in computing systems - CHI '14. (2014), 371-380.

[20] Casiez, G. and Roussel, N. 2011. No more bricolage!: Methods and tools to characterize, replicate and compare pointing transfer functions. UIST (2011), 603-614.

[21] Croteam 2017. Serious Sam: The First Encounter VR.

[22] Croteam 2017. Serious Sam Fusion 2017 Editor.

[23] Desurvire, H. and Wiberg, C. 2009. Game Usability Heuristics ( PLAY ) For Evaluating and Designing Better Games : The Next Iteration. Online Communities and Social Computing (2009), 557-566.

[24] Farmani, Y. 2018. Discrete Viewpoint Control to Reduce Cybersickness in Virtual Environments.

[25] Farmani, Y. and Interaction, H.C. 2018. Discrete Viewpoint Control to Reduce Cybersickness in Virtual Environments Affairs in partial fulfillment 
of the requirements for the degree of Master of Computer Science. (2018).

[26] Farmani, Y. and Teather, R.J. 2018. Player performance with different input devices in virtual reality first-person shooter games. Spatial User Interaction (2018).

[27] Farmani, Y. and Teather, R.J. 2017. Player performance with different input devices in virtual reality first-person shooter games. SUI (2017), 165.

[28] Farmani, Y. and Teather, R.J. 2018. Viewpoint snapping to reduce cybersickness in virtual reality. Graphics Interface (2018), 159-166.

[29] Fuhrmann, A. et al. 1998. Strolling through Cyberspace with Your Hands in Your Pockets: Head Directed Navigation in Virtual Environments. EUROGRAPHICS (1998), 216-227.

[30] Funk, M. et al. 2019. Assessing the Accuracy of Point \& Teleport Locomotion with Orientation Indication for Virtual Reality using Curved Trajectories. CHI (2019).

[31] Gerling, K.M. et al. 2011. Measuring the impact of game controllers on player experience in FPS games. MindTrek (2011), 83-87.

[32] Griffin, N.N. and Folmer, E. 2019. Out-of-body Locomotion: Vectionless Navigation with a Continuous Avatar Representation. (2019), 1-8. DOI:https://doi.org/10.1145/3359996.3364243.

[33] Grossman, T. et al. 2009. A survey of software learnability: Metrics, methodologies and guidelines. Conference on Human Factors in Computing Systems - Proceedings. (2009), 649-658. DOI:https://doi.org/10.1145/1518701.1518803.

[34] Harris, J. 2008. Game Design Essentials: 20 Unusual Control Schemes. 


\section{Gamasutra.}

[35] ID Software 1996. Quake.

[36] Ilves, M. et al. 2014. Head movement and facial expressions as game input. Entertainment Computing. 5, 3 (2014), 147-156.

[37] Jennett, C. et al. 2008. Measuring and defining the experience of immersion in games. International Journal of Human-Computer Studies. 66, 9 (Sep. 2008), 641-661.

[38] Johnson, D. and Gardner, J. 2010. Personality, Motivation and Video Games. Proceedings of the 22nd Conference of the Computer-Human Interaction Special Interest Group of Australia on Computer-Human Interaction - OZCHI '10. (2010), 276.

DOI:https://doi.org/10.1145/1952222.1952281.

[39] Kitson, A. et al. 2017. Comparing leaning-based motion cueing interfaces for virtual reality locomotion. 2017 IEEE Symposium on $3 D$ User Interfaces, 3DUI 2017 - Proceedings. (2017), 73-82.

DOI:https://doi.org/10.1109/3DUI.2017.7893320.

[40] Klochek, C. and Mackenzie, I.S. 2006. Performance measures of game controllers in a three-dimensional environment. Proc. GI (2006), 73-79.

[41] Kulshreshth, A. and Laviola, J.J. 2013. Evaluating performance benefits of head tracking in modern video games. Spatial User Interaction (2013), 5360.

[42] Lampton, D.R. et al. 1995. The Virtual Environment Performance Assessment Battery: Development and Evaluation.

[43] Limperos, A.M. et al. 2011. Gaming across different consoles: exploring the 
influence of control scheme on game-player enjoyment. Cyberpsychology, Behavior and Social Networking. 14, 6 (2011), 345-350.

DOI:https://doi.org/10.1089/cyber.2010.0146.

[44] Lombard, M. and Ditton, T. 1997. At the heart of it all: the concept of presence. Journal of Computer-Mediated Communication. 3, 2 (1997).

[45] Lu, W. Evolution of Video Game Controllers : How Simple Switches Lead to the Development of the Joystick and the Directional Pad By.

[46] Lugrin, J.-L. et al. 2012. Are immersive FPS games enjoyable? Virtual Reality Software and Technology (2012), 199.

[47] Lugrin, J. et al. 2013. Immersive FPS games: user experience and performance. ImmerseveMe (2013), 7-12.

[48] Macaranas, A. and Antle, A. 2013. The Effects of Intuitive Interaction Mappings on the Usability of Body - based Interfaces by.

[49] MacKenzie, I.S. 2013. Human-computer Interaction: An Empirical Research Perspective.

[50] MacKenzie, I.S. 2013. Human-Computer Interaction: An Empirical Research Perspective. Elsevier.

[51] Martel, E. et al. 2015. Diving Head-First into Virtual Reality- Evaluating HMD Control Schemes for VR Games. Foundations of Digital Games (2015), $1-4$.

[52] Martel, E. et al. 2015. Diving Head-First into Virtual Reality- Evaluating HMD Control Schemes for VR Games. Foundations of Digital Games. Fdg (2015), $1-4$.

[53] Martel, E. and Muldner, K. 2017. Controlling VR games: control schemes 
and the player experience. Entertainment Computing. 21, (2017).

DOI:https://doi.org/10.1016/j.entcom.2017.04.004.

[54] Mäyrä, F. and Ermi, L. 2005. Fundamental components of the gameplay experience: Analysing immersion. DiGRA (2005), 88-115.

[55] McEwan, M.W. et al. 2014. Natural mapping and intuitive interaction in videogames. Proceedings of the first ACM SIGCHI annual symposium on Computer-human interaction in play - CHI PLAY'14. (2014), 191-200. DOI:https://doi.org/10.1145/2658537.2658541.

[56] Mine, M.R. 1995. Virtual Environment Interaction Techniques, UNC Chapel Hill Computer Science Technical Report TR95-O18.

[57] Muldner, K. and Conati, C. 2010. Scaffolding meta-cognitive skills for effective analogical problem solving via tailored example selection. International Journal of Artificial Intelligence in Education. 20, (2010), 99-136. DOI:https://doi.org/10.3233/JAI-2010-0004.

[58] Nabiyouni, M. et al. 2015. Comparing the performance of natural, seminatural, and non-natural locomotion techniques in virtual reality. Virtual Reality. (2015), 243-244. DOI:https://doi.org/10.1109/VR.2015.7223386.

[59] Natapov, D. et al. 2009. ISO 9241-9 evaluation of video game controllers. GI (2009), 223-230.

[6o] Oculus Rift 2019. PC SDK Developer Guide.

[61] Oculus VR 2013. Oculus Rift. 2013.

[62] Oculus VR 2014. Oculus Rift Development Kit 2.

[63] Pausch, R. et al. 1993. A User Study Comparing Head-Mounted and Stationary Displays. VR (1993), 41-45. 
[64] Ramcharitar, A. and Teather, R.J. 2017. A Fitts' law evaluation of video game controllers: Thumbstick, touchpad and gyrosensor. CHI (2017), $2860-2866$.

[65] Ruddle, R.A. et al. 2013. Learning to walk in virtual reality. $A C M$ Transactions on Applied Perception. 10, 2 (2013), 11-35.

[66] Ruddle, R.A. et al. 2013. Learning to Walk in Virtual Reality. $A C M$ Transactions on Applied Perception. 10, 2 (2013).

[67] Skalski, P. et al. 2011. Mapping the road to fun: natural video game controllers, presence, and game enjoyment. New Media \& Society. 13, 2 (2011), 224-242.

[68] Sko, T. et al. 2013. Studying a head tracking technique for first-personshooter games in a home setting. Interact (2013), 246-263.

[69] Sko, T. and Gardner, H.J. 2009. Head tracking in first-person games: interaction using a web camera. Interact (2009), 342-355.

[70] Slater, M. 2003. A Note on Presence Terminology. Presence Connect. 3, 3 (2003), 1-5.

[71] Soukoreff, R.W. and MacKenzie, I.S. 2004. Towards a standard for pointing device evaluation, perspectives on 27 years of Fitts' law research in HCI. International Journal of Human Computer Studies. 61, 6 (2004), 751-789. DOI:https://doi.org/10.1016/j.ijhcs.2004.09.001.

[72] Standardization, I.O. for 2015. ISO 9001:2015.

[73] Steuer, J. 1992. Defining Virtual Reality : Dimensions Determining Telepresence. Journal of Communication. 42, 4 (1992), 73-95.

[74] Tan, C.T. et al. 2015. Exploring gameplay experiences on the Oculus Rift. 
CHI PLAY (2015), 253-263.

[75] Tashakkori, A. and Creswell, J.W. 2007. Exploring the Nature of Research Questions in Mixed Methods Research. Journal of Mixed Methods Research. 1, 3 (2007), 207-211.

[76] Teather, R.J. and Stuerzlinger, W. 2011. Pointing at 3D targets in a stereo head-tracked virtual environment. 3DUI (2011), 87-94.

[77] Turchet, L. 2015. Designing presence for real locomotion in immersive virtual environments: an affordance-based experiential approach. Virtual Reality. 19, 3-4 (2015), 277-290. DOI:https://doi.org/10.1007/s10055015-0267-3.

[78] Unsöld, M. 2018. Measuring Learnability in Human-Computer Interaction. Ulm University. (2018), 112.

[79] Usoh, M. et al. 1999. Walking > walking-in-place > flying, in virtual environments. Proceedings of the 26th annual conference on Computer graphics and interactive techniques - SIGGRAPH '99. (1999), 359-364. DOI:https://doi.org/10.1145/311535·311589.

[80] Valve Corporation 2004. Half-Life 2: Deathmatch.

[81] Valve Corporation 2014. Source SDK.

[82] Volbracht, S. and Domik, G. 200o. Developing Effective Navigation Techniques in Virtual 3D-Environments. Virtual Environments (2000), $55^{-64}$

[83] Wang, S. et al. 2006. Face tracking as an augmented input in video games: enhancing presence, role-playing and control. CHI (2006), 1097-1106.

[84] Ware, C. and Osborn, S. 1990. Exploration and Virtual Camera Control in 
Virtual Three Dimensional Environments. Proc. I3D (1990), 175-183.

[85] Wilson, P.T. et al. 2016. VR Locomotion: Walking > Walking in Place > Arm Swinging. VRCAI (2016), 243-249.

[86] Yoon, J.-W. et al. 2010. Enhanced user immersive experience with a virtual reality based FPS game interface. Computational Intelligence and Games (Aug. 2010), 69-74.

[87] Zaranek, A. et al. 2014. Performance of modern gaming input devices in first-person shooter target acquisition. CHI (2014), 1495-1500.

[88] Al Zayer, M. et al. 2018. Virtual Locomotion: a Survey. IEEE Transactions on Visualization and Computer Graphics. (2018), 1-20. DOI:https://doi.org/10.1109/TVCG.2018.2887379.

[89] Zhou, Z. et al. 2007. User studies of a multiplayer first person shooting game with tangible and physical interaction. Virtual Reality (2007). 